TRANSACTIONS OF THE

AMERICAN MATHEMATICAL SOCIETY

Volume 357, Number 2, Pages 617-667

S 0002-9947(04)03460- 9

Article electronically published on April 16, 2004

\title{
ON STRUCTURALLY STABLE DIFFEOMORPHISMS WITH CODIMENSION ONE EXPANDING ATTRACTORS
}

\author{
V. GRINES AND E. ZHUZHOMA
}

\begin{abstract}
We show that if a closed $n$-manifold $M^{n}(n \geq 3)$ admits a structurally stable diffeomorphism $f$ with an orientable expanding attractor $\Omega$ of codimension one, then $M^{n}$ is homotopy equivalent to the $n$-torus $T^{n}$ and is homeomorphic to $T^{n}$ for $n \neq 4$. Moreover, there are no nontrivial basic sets of $f$ different from $\Omega$. This allows us to classify, up to conjugacy, structurally stable diffeomorphisms having codimension one orientable expanding attractors and contracting repellers on $T^{n}, n \geq 3$.
\end{abstract}

\section{INTRODUCTION}

Structurally stable diffeomorphisms form an important class of dynamical systems. They comprise an open nonempty set in the space of diffeomorphisms on any closed manifold. For this reason, it is of interest to understand on what manifolds a given class of structurally stable diffeomorphisms satisfying additional conditions exists. In 1, it was proved that Anosov diffeomorphisms on a compact manifold are structurally stable. Several papers have investigated the relationship between the topology of underlying manifolds and the dynamics of codimension one Anosov diffeomorphisms. Franks [12] and Newhouse 37] show that any codimension one Anosov diffeomorphism is conjugate to a hyperbolic torus automorphism (as a consequence, a manifold admitting such diffeomorphisms is homeomorphic to the torus $\left.T^{n}, n \geq 2\right)$. A simple proof of this Franks-Newhouse theorem that uses foliation theory techniques was recently obtained in [25].

An important class of dynamical systems is made up of the diffeomorphisms satisfying Smale's Axiom A [53], the so-called $A$-diffeomorphisms. Given such a diffeomorphism, its recurrent behaviour is captured in its nonwandering set, which can be decomposed into invariant topologically transitive pieces (basic sets). One remarkable type of basic sets is an expanding attractor, introduced by Williams [58], [60]. Such an attractor has a complicated topological structure that is combined with a nontrivial recurrence (see section[1). A structurally stable $A$-diffeomorphism of $T^{n}$ with an orientable codimension one expanding attractor can be obtained by Smale's surgery ([53, pp. 788-789); it is a codimension one Anosov diffeomorphism (the orientability of an expanding attractor, roughly speaking, means the following: given any arc of a stable manifold and a codimension one unstable manifold, the index of their intersection is the same at every point of intersection; see section 1 for details). Such diffeomorphisms are called DA-diffeomorphisms.

Received by the editors March 15, 2001 and, in revised form, April 10, 2003 and July 10, 2003. 2000 Mathematics Subject Classification. Primary 37D20; Secondary 37C70, 37C15.

This research was partially supported by the RFFI grant 02-01-00098. 
Must any structurally stable $C^{\infty}$-diffeomorphism with an orientable codimension one expanding attractor look like a $D A$-diffeomorphism? This is just the question we consider here for dimensions $\operatorname{dim} M^{n}=n \geq 3$. Ideologically we follow the papers [12] and [37. Note that the answer is well-known for the dimension $n=2$. Namely, starting with a $D A$-diffeomorphism of $T^{2}$, one can construct structurally stable diffeomorphisms with codimension one orientable expanding attractors on any closed orientable surface of genus $g \geq 1$. (See, e.g., [5, 44, 49. There is a careful construction of $D A$-diffeomorphism on $T^{2}$ in [31] and [48.)

There are two ways to classify expanding attractors. The first way is to ask, when are the restrictions of two diffeomorphisms to their expanding attractors conjugate? This type of classification was obtained by Williams [60], Theorem A, who proved that the restriction of a diffeomorphism on an expanding attractor of any dimension is conjugate to the shift map of an $n$-solenoid. The second way is to ask, when are two diffeomorphisms conjugate in neighborhoods of their attractors? The authors of [50] constructed two diffeomorphisms $f$ and $g$ with attractors $\Omega_{f}$ and $\Omega_{g}$ such that $f: \Omega_{f} \rightarrow \Omega_{f}$ is conjugate to $g: \Omega_{g} \rightarrow \Omega_{g}$ but there is not even a homeomorphism from a neighborhood of $\Omega_{f}$ to a neighborhood of $\Omega_{g}$ taking $\Omega_{f}$ to $\Omega_{g}$. Thus, these two ways of classification are different. Here we consider the second type of classification. In [15], [16, [17], [18] structurally stable diffeomorphisms with one-dimensional expanding attractors on orientable closed surfaces of genus $g \geq 0$ are classified. In [7] structurally stable diffeomorphisms on orientable closed surfaces of genus $g \geq 0$ are classified. Let us mention the combinatorial description obtained in 61, 62 for one-dimensional expanding attractors on closed surfaces (orientable or non-orientable).

It is well-known that a codimension one basic set (i.e., the topological dimension of the basic set equals $n-1$ ) is either an attractor or a repeller. As we know, Smale's question (see [53], p. 785) of whether such nontrivial basic set that is not a codimension one compact submanifold is locally the product of a Cantor set and a manifold is unsolved up to now (in the "codimension one" case). Guckenheimer [22 constructed a structurally stable diffeomorphism of the 5 -sphere with a codimension four basic set that is not locally the product of a Cantor set and an interval. Farrell and Jones [11] constructed the orientable basic set of codimension one that is not the total space of a fiber bundle with a manifold for a base space and a Cantor set for fiber. Other interesting examples can be found in 30 and 44 .

If a codimension one basic set is an expanding attractor or contracting repeller, then it is locally the product of a Cantor set and a codimension one hyperplane (recall that an expanding attractor has topological dimension equal to the dimension of unstable manifolds of points of the attractor. A contracting repeller of a diffeomorphism $f$ is an expanding attractor for $f^{-1}$ ) [43], 60]. In this paper we consider a codimension one basic set that is an expanding attractor or contracting repeller. Certainly, one can consider a contracting repeller instead of an expanding attractor, and vice versa. Therefore we use the notion pseudotame basic set, meaning an expanding attractor or contracting repeller.

As we mentioned above, any closed orientable surface of genus $g \geq 1$ admits codimension one orientable pseudotame basic sets [5], [44, 49], 53]. Moreover, any such surface admits a structurally stable diffeomorphism with orientable pseudotame basic sets. As to closed $n$-manifolds for $n \geq 3$, the first example of a structurally stable $D A$-diffeomorphism of $T^{3}$ with a codimension one orientable 
expanding attractor was constructed carefully by Franks and Robinson 13]. This construction was generalized by Plykin [45] for $n \geq 3$ in the framework of the topological classification of codimension one pseudotame basic sets. In [13], one constructs a diffeomorphism $f$ of the connected sum $T^{3} \sharp T^{3}$ that is quasi-Anosov but not Anosov. Actually, $f$ has two orientable codimension one pseudotame basic sets, one of them an expanding attractor and the other a contracting repeller. Examination of this example shows that $f$ is not structurally stable, because there are intersections of one-dimensional stable and unstable manifolds of points of the expanding attractor and the contracting repeller respectively, and thus the strong transversality condition fails. This raises the natural question of whether there exist structurally stable diffeomorphisms with orientable codimension one pseudotame basic sets on closed $n$-manifolds $(n \geq 3)$ different from $T^{n}$.

Let us mention some topological obstructions to manifolds supporting codimension one pseudotame basic sets. In [32], one proved that if a closed $n$-manifold $M^{n}$, $n \geq 3$, admits a codimension one expanding attractor (orientable or not), then $M^{n}$ has a nontrivial fundamental group. In particular, there are no such diffeomorphisms of the $n$-sphere $S^{n}, n \geq 3$. The more general result was obtained later in 45], where one proved that the fundamental group $\pi_{1}\left(M^{n}\right)$ contains a subgroup isomorphic to the integer lattice $\mathbb{Z}^{n}$. To be precise, Plykin [45] showed that if $f$ has $k \geq 1$ copies of codimension one pseudotame basic sets (orientable or not), then $\pi_{1}\left(M^{n}\right)$ contains the free product of $k$ copies of $\mathbb{Z}^{n}$ (for orientable basic sets, this result was obtained in 63]). Plante [42 proved that an orientable codimension one expanding attractor defines a nontrivial element of the first homology group $H_{1}\left(M^{n}\right)$. Sullivan and Williams [56] showed that the real Cech homology of an oriented attractor (of any codimension) in its top dimension is nontrivial and finite dimensional.

Our following theorem describes the homotopy type of a closed manifold $M^{n}$ $(n \geq 3)$ admitting a structurally stable diffeomorphism with an orientable pseudotame basic set of codimension one. Recall that in our theorems we consider diffeomorphisms to be $C^{\infty}$ diffeomorphisms, unless stated otherwise. From now on, the nontriviality of a basic set means that the basic set is not a periodic orbit.

Theorem 7.1. Suppose $f$ is a structurally stable diffeomorphism of a closed $n$ manifold $M^{n}(n \geq 3)$ and $\Omega$ is a codimension one orientable expanding attractor of $f$. Then:

1) $M^{n}$ is homotopy equivalent to the $n$-torus $T^{n}$. If $n \neq 4$, then $M^{n}$ is homeomorphic to $T^{n}$.

2) $\Omega$ is the only nontrivial basic set of $f$.

3) $f$ has at least one repelling periodic point.

If $x$ is a periodic point of $f$, then the index at $x$ is defined to be the dimension of the unstable manifold $W^{u}\left(f^{p}(x)\right)$, where $p$ is the period of $x$. The index of a periodic orbit equals the index of any point of it.

Corollary 17.1. Suppose $f$ is a structurally stable diffeomorphism of a closed nmanifold $M^{n}(n \geq 3)$ having an orientable expanding attractor $\Omega$ of codimension one. Then the spectral decomposition of $f$ consists of $\Omega$, and a finite nonzero number of repelling periodic orbits of index $n$, and a finite number (maybe zero) of periodic saddle orbits of index $n-1$. 
Corollary [7.3, Suppose $f$ is an A-diffeomorphism of a closed n-manifold $M^{n}$ $(n \geq 3)$ having an orientable expanding attractor of codimension one $\Omega$. Then the following conditions imply that $f$ is not structurally stable:

1) $f$ has a nontrivial basic set different from $\Omega$.

2) $M^{n}$ is not homotopy equivalent to the $n$-torus.

After Theorem 17.1, it is natural to classify, up to conjugacy, structurally stable diffeomorphisms with orientable pseudotame basic sets of codimension one on the torus $T^{n}$. For these diffeomorphisms we introduce the complete invariant of conjugacy, which is a finite collection of periodic orbits of some hyperbolic torus automorphism endowed with presigned natural numbers. Such a collection is called a data set of the hyperbolic automorphism (for details, see section [8).

Theorem 8.2. Suppose $f_{1}, f_{2}: T^{n} \rightarrow T^{n}$ are structurally stable diffeomorphisms having orientable expanding attractors of codimension one $\Omega_{1}$ and $\Omega_{2}$ respectively. Then $f_{1}$ and $f_{2}$ are conjugate if and only if the data sets $\mathcal{D}\left(f_{1}, h_{1}\right)$ and $\mathcal{D}\left(f_{2}, h_{2}\right)$ are equivalent, where $h_{i}: T^{n} \rightarrow T^{n}(i=1,2)$ are continuous maps homotopic to the identity and such that $h_{i} \circ f_{i}=A_{i} \circ h_{i},\left(f_{i}\right)_{*}=\left(A_{i}\right)_{*}$.

Theorem 8.3. Let $A$ be a codimension one hyperbolic automorphism of $T^{n}$ such that the stable manifolds of $A$ are one-dimensional. Given an admissible data set $\left\{O_{j}, n_{j}\right\}_{j=1}^{r}$ of $A$, there is a structurally stable diffeomorphism $f: T^{n} \rightarrow T^{n}$ having an orientable expanding attractor of codimension one and such that $\mathcal{D}(f, h)=$ $\left\{O_{j}, n_{j}\right\}_{j=1}^{r}$, where $h: T^{n} \rightarrow T^{n}$ is a continuous map homotopic to the identity with $h \circ f=A \circ h$ and $f_{*}=A_{*}$.

The first example of a codimension one non-orientable expanding attractor $\Omega$ on the two-sphere $S^{2}$ was constructed by Plykin [44. He constructed a structurally stable diffeomorphism $f: S^{2} \rightarrow S^{2}$ with so-called 1-bunches. Roughly speaking, there exists a component of $S^{2}-\Omega$ whose accessible boundary consists of a unique (one-dimensional) unstable manifold of a periodic point (see section 1). In [45], codimension one non-orientable expanding attractors with 1-bunches on open $n$-manifolds, $n \geq 3$, were constructed (in the same paper, Plykin classified up to conjugacy diffeomorphisms which are restricted to some neighborhoods of codimension one expanding attractors). Bearing these examples in mind, a codimension one expanding attractor $\Omega$ is called a codimension one Plykin attractor whenever $\Omega$ has 1-bunches.

The following theorem was proved in collaboration with V. Medvedev.

Theorem 7.3. Let $f: M^{3} \rightarrow M^{3}$ be a structurally stable diffeomorphism of a closed 3-manifold $M^{3}$. Then the spectral decomposition of $f$ contains neither codimension one non-orientable expanding attractors nor codimension one non-orientable contracting repellers. In particular, $f$ has no codimension one Plykin attractors.

Corollary [8.1. Suppose $f_{1}, f_{2}: T^{3} \rightarrow T^{3}$ are structurally stable diffeomorphisms having expanding attractors of codimension one $\Omega_{1}$ and $\Omega_{2}$ respectively. Then $f_{1}$ and $f_{2}$ are conjugate if and only if the data sets $\mathcal{D}\left(f_{1}, h_{1}\right)$ and $\mathcal{D}\left(f_{2}, h_{2}\right)$ are equivalent, where $h_{i}: T^{3} \rightarrow T^{3}(i=1,2)$ are continuous maps homotopic to the identity and such that $h_{i} \circ f_{i}=A_{i} \circ h_{i}$ and $\left(f_{i}\right)_{*}=\left(A_{i}\right)_{*}$.

This paper is organized as follows. In section 1 we give the main definitions. We formulate for convenience some well-known results on codimension one basic sets. 
We generalize some of these results and give sketches of proofs. In section 2 given a codimension one expanding attractor, we construct characteristic spheres. Here we actually follow the construction of Newhouse [37] for a nontransitive Anosov diffeomorphism. In sections 3 and 4 we prove some technical results we will need later. In section 5 we study the structure of the universal covering for $M^{n}$. In this section we prove the following theorem, which is crucial for the proof of item 1) of Theorem 7.1.

Theorem [5.1, Let $f$ be an A-diffeomorphism of a closed $n$-manifold $M^{n}(n \geq 3)$ and let $\Omega$ be an orientable expanding attractor of codimension one. Suppose that each characteristic sphere $S_{p_{i} q_{i}}$ of $\Omega$ bounds an $n$-ball $E_{p_{i} q_{i}} \subset M^{n}$. Then:

1) Any basic set different from $\Omega$ belongs to the union $\bigcup_{i=1}^{k} E_{p_{i} q_{i}}$, where $p_{i}$, $q_{i}$ range over all pairs of associated boundary periodic points of $\Omega$.

2) $\Omega$ is the only orientable codimension one pseudotame basic set of $f$.

3) The universal covering $\bar{M}$ of $M^{n}$ is homeomorphic to Euclidean space $\mathbb{R}^{n}$.

4) The fundamental group $\pi_{1}\left(M^{n}\right)$ is a free abelian finitely generated group. In particular, $M^{n}$ is homotopy equivalent to the $n$-torus $T^{n}$.

In section [6] we prove the following theorem, needed for the proof of the remaining items of Theorem 7.1 In a sense, this theorem can be considered as a sufficient condition for the nonstability of $f$.

Theorem 6.2, Let $f$ be an A-diffeomorphism of a closed $n$-manifold $M^{n}(n \geq 3)$ and $\Omega$ an orientable expanding attractor of codimension one. Suppose $\Omega^{\prime} \neq \Omega$ is a nontrivial basic set of $f$ such that $W^{u}\left(\Omega^{\prime}\right) \cap W^{s}(\Omega) \neq \emptyset$. Then $f$ is not structurally stable.

Theorems 7.1 and 7.3 are proved in section 7 . The classification Theorems 8.2 and 8.3 are proved in section 8

\section{MAin Definitions}

We begin by recalling several definitions. Further details may be found in [31, [38], [48, [53].

Let $f$ be a $C^{\infty}$ diffeomorphism of a closed manifold $M^{n}$ endowed with some Riemannian metric $d$. $f$ is said to be an A-diffeomorphism if its nonwandering set $N W(f)$ is hyperbolic and periodic points are dense in $N W(f)$ [53. The stable manifold $W^{s}(x)$ of a point $x \in N W(f)$ is defined to be the set of points $y \in M^{n}$ such that $d\left(f^{i} x, f^{i} y\right) \rightarrow 0$ as $i \rightarrow+\infty$. The unstable manifold $W^{u}(x)$ of $x$ is the stable manifold of $x$ for the diffeomorphism $f^{-1}$. We shall consider a stable or unstable manifold to be an immersed submanifold of $M^{n}$. Stable and unstable manifolds are called invariant manifolds. It is well known [27] that invariant manifolds depend continuously on initial conditions on compact sets. By definition, let $W_{\varepsilon}^{s}(x) \subset$ $W^{s}(x)$ (resp. $W_{\varepsilon}^{u}(x) \subset W^{u}(x)$ ) be the $\varepsilon$-neighborhood of $x$ in the intrinsic topology of the manifold $W^{s}(x)$ (resp. $W^{u}(x)$ ), where $\varepsilon>0$.

Let Diff ${ }^{1}\left(M^{n}\right)$ be the space of $C^{1}$ diffeomorphisms on $M^{n}$ endowed with the uniform $C^{1}$ topology [26]. A diffeomorphism $f$ is said to be structurally stable if there exists a neighborhood $U$ of $f$ in $\operatorname{Diff}^{1}\left(M^{n}\right)$ such that every $g \in U$ is conjugate to $f$.

Let $f$ be an $A$-diffeomorphism. We say that $f$ satisfies the strong transversality condition if for every $x, y \in N W(f), W^{s}(x)$ is transverse to $W^{u}(y)$. According 
to Mañé-Robinson's theorem [35], 47], a diffeomorphism $f$ is structurally stable if and only if $f$ is an $A$-diffeomorphism satisfying the strong transversality condition.

The spectral decomposition theorem says that the nonwandering set $N W(f)$ of an $A$-diffeomorphism $f$ is a finite union of pairwise disjoint $f$-invariant closed sets $\Omega_{1}, \ldots, \Omega_{k}$ such that every restriction $\left.f\right|_{\Omega_{i}}$ is topologically transitive. These $\Omega_{i}$ are called the basic sets of $f$. Since $f$ is transitive on each basic set $\Omega_{i}$, it follows that the restrictions of the bundles $E^{s}, E^{u}$ to $\Omega_{i}$ have constant dimensions. The dimension $\operatorname{dim} E_{\Omega_{i}}^{u}=\operatorname{dim} E_{x}^{u}, x \in \Omega_{i}$, is called Morse's index of $\Omega_{i}$.

A basic set is nontrivial if it is not a periodic isolated orbit. Due to [2] and [8], every nontrivial basic set $\Omega_{i}$ is a union of pairwise disjoint closed subsets $\Omega_{i 1}, \ldots$, $\Omega_{i h}$ such that

$$
f^{h}\left(\Omega_{i j}\right)=\Omega_{i j}, \quad f\left(\Omega_{i j}\right)=\Omega_{i, j+1}, \text { where } \Omega_{i, h+1}=\Omega_{i 1} \quad(1 \leq j \leq h),
$$

and each of the intersections $W_{x}^{s} \cap \Omega_{i j}$ and $W_{x}^{u} \cap \Omega_{i j}$ is dense in $\Omega_{i j}$ for any point $x \in \Omega_{i j}$. These $\Omega_{i j}$ are called $C$-dense components of $\Omega_{i}$. If $\Omega_{i}$ has a unique $C$-dense component, then we will say that $\Omega_{i}$ is a $C$-dense basic set.

For any $x \in \Omega, W^{u}(x)$ and $W^{s}(x)$ are immersed submanifolds such that

$$
\operatorname{dim} W^{u}(x)+\operatorname{dim} W^{n}(x)=n .
$$

Moreover, $W^{u}(x)$ and $W^{s}(x)$ are homeomorphic to Euclidean space of the corresponding dimension. Therefore both $W^{u}(x)$ and $W^{s}(x)$ are endowed with a normal and intrinsic orientation. Hence one can define the index of intersection at each point of $W^{u}(x) \cap W^{s}(x)$ [26]. Following [14] and [15], we call a basic set $\Omega$ orientable if for any $\alpha>0$ and $\beta>0$ the index of $W_{x, \alpha}^{s} \cap W_{x, \beta}^{u}$ does not depend on a point of intersection.

Following [60], a basic set $\Omega$ is called a codimension one expanding attractor if $\operatorname{dim} E_{\Omega}^{u}=\operatorname{dim} \Omega=n-1$.

Due to [43] and 60], an expanding attractor $\Omega$ of codimension one consists of $(n-1)$-dimensional unstable manifolds $W_{x}^{u}, x \in \Omega$ (thus, $\operatorname{dim} E_{\Omega}^{s}=1$ ). Moreover, $\Omega$ is locally homeomorphic to the product of $(n-1)$-dimensional Euclidean space and a Cantor set. For given points $x, y \in W^{s}(z)(z \in \Omega)$, denote by $(x, y)^{s}$ (resp. $\left.[x, y]^{s}\right)$ an open (resp. closed) arc of $W^{s}(z)$ with the endpoints $x, y$.

For the reader's convenience, we give sketches of proofs of some results on codimension one basic sets and codimension one expanding attractors, mainly obtained by Grines [14], 15], Newhouse [37], Plykin [44, and Williams [60]. The reader who is familiar with the cited papers can skip this part of the section.

Lemma 1.1. Let $\Omega$ be a basic set of index $n-1$ of an A-diffeomorphism $f: M^{n} \rightarrow$ $M^{n}, n \geq 2$. If $x \in \Omega$ is a nonperiodic point, then both components of $W^{s}(x)-x$ intersect $W^{u}(\Omega)$.

Proof. Let $x_{i}=f^{n_{i}}(x) \rightarrow x_{*} \in \Omega$ be a convergent sequence $\left(n_{i} \rightarrow+\infty\right)$. First we suppose that $W^{u}(x)$ does not contain periodic points; then we can assume that the sets $W^{u}\left(f^{n_{i}}(x)\right)=f^{n_{i}}\left(W^{u}(x)\right)$ are disjoint. Due to the local product structure, $W_{\varepsilon}^{s}\left(x_{2}\right) \cap W_{\varepsilon}^{u}\left(x_{j}\right)$ is a single point of $\Omega$ in some neighborhood of $x_{*}$, where $j=1,3$. Without loss of generality we can assume that the points $W_{\varepsilon}^{s}\left(x_{2}\right) \cap W_{\varepsilon}^{u}\left(x_{1}\right)$ and $W_{\varepsilon}^{s}\left(x_{2}\right) \cap W_{\varepsilon}^{u}\left(x_{3}\right)$ belong to different components of $W_{\varepsilon}^{s}\left(x_{2}\right)-x_{2}$, because the $W^{u}\left(x_{i}\right)$ are disjoint. Hence both components of $W^{s}(x)-x$ intersect $\Omega$.

Suppose now that $W^{u}(x)$ contains a periodic point, say $x_{0} \in \Omega$. Without loss of generality we can assume that $x_{0}$ is a fixed point. Since the map $\left.f\right|_{W^{u}\left(x_{0}\right)}$ expands 
the invariant manifold $W^{u}\left(x_{0}\right)$, it follows that there is a sequence $n_{i} \rightarrow+\infty$ such that the distance between $f^{n_{i}}(x)$ and $f^{n_{j}}(x)$ in a metric of $W^{u}(x)$ is more than $\varepsilon$ whenever $i \neq j$. We can assume that the sequence $f^{n_{i}}(x)$ converges to some point $x_{*} \in \Omega$. In this case, the proof is similar.

Taking into account that $W^{u}(\Omega)=\Omega$ whenever $\Omega$ is a codimension one expanding attractor, we get the following lemma as a consequence.

Lemma 1.2. Let $\Omega$ be a codimension one expanding attractor (orientable or not) of an A-diffeomorphism $f: M^{n} \rightarrow M^{n}, n \geq 2$. Suppose $x \in \Omega$ is a nonperiodic point of $f$. Then both components of $W^{s}(x)-x$ intersect $\Omega$.

The following lemma is a sharpening of Lemma 1.1

Lemma 1.3. Let $\Omega$ be a basic set of index $n-1$ of an A-diffeomorphism $f: M^{n} \rightarrow$ $M^{n}, n \geq 2$. Suppose $W^{u}(x)(x \in \Omega)$ does not contain periodic points. Then both components of $W_{\delta}^{s}(x)-x$ intersect $W^{u}(\Omega)$ for any $\delta>0$.

Proof. Let $f^{n_{i}}(x) \rightarrow x_{*}\left(n_{i} \rightarrow-\infty\right)$ be a convergent sequence. Then $W^{u}\left(f^{n_{i}}(x)\right)=$ $f^{n_{i}}\left(W^{u}(x)\right)$ are pairwise disjoint. Due to the local product structure in some neighborhood of $x_{*}$, both components of $W_{\varepsilon}^{s}\left(f^{n_{i}}(x)\right)-f^{n_{i}}(x)$ intersect $W^{u}(\Omega)$. Since

$$
\lim _{n_{i} \rightarrow-\infty} \operatorname{diam} f^{-n_{i}}\left(W_{\varepsilon}^{s}\left(f^{n_{i}}(x)\right)\right) \rightarrow 0,
$$

it follows that both components of $W_{\delta}^{s}(x)-x$ intersect $W^{u}(\Omega)$ for any $\delta>0$.

Lemma 1.4. Suppose $\Omega$ is a codimension one expanding attractor (orientable or not) of an A-diffeomorphism $f: M^{n} \rightarrow M^{n}, n \geq 2$. Then there exists a periodic point $p \in \Omega$ of $f$ such that one of the components of $W^{s}(p)-p$ does not intersect $\Omega$.

Proof. Assume the converse. Then by Lemma 1.2, both components of $W^{s}(x)-x$ intersect $\Omega$ for any $x \in \Omega$. Let us show that $W^{s}(x) \subset \Omega$. Take a convergent sequence $f^{-n_{i}}(x) \rightarrow y \in \Omega, n_{i} \rightarrow+\infty$. By assumption, both components of $W^{s}(y)-y$ intersect $\Omega$. As a consequence, both components of $W_{\varepsilon}^{s}\left(f^{-n_{i}}(x)\right)-f^{n_{i}}(x)$ intersect $\Omega$ for some $\varepsilon>0$. Since $f$ contracts every stable manifold, we see that both components of $W_{\delta}^{s}(x)-x$ intersect $\Omega$ for any $\delta>0$. Hence $W^{s}(x) \subset \Omega$, because $\Omega$ is closed. This implies that $\Omega=M^{n}$, which contradicts the fact that $\Omega$ is an attractor. This concludes the proof.

Later on, we specify $\Omega$ to be a codimension one expanding attractor of an $A$-diffeomorphism $f: M^{n} \rightarrow M^{n}, n \geq 2$, unless otherwise arranged. Following [14] and [15], $p \in \Omega$ is called a boundary point if at least one component of $W^{s}(p)-p$ does not intersect $\Omega$. A point is interior if it is not a boundary point. Due to Lemma 1.4, boundary points exist. By Lemma 1.2 any boundary point $p \in \Omega$ is periodic.

Lemma 1.5. Let $p$ be a boundary point of a codimension one expanding attractor $\Omega$. Then there is a unique component of $W^{s}(p)-p$, denoted by $W_{\emptyset}^{s}(p)$, that does not intersect $\Omega$. Moreover, the other component

$$
W_{\infty}^{s}(p) \stackrel{\text { def }}{=} W^{s}(p)-\left(p \cup W_{\emptyset}^{s}(p)\right)
$$

contains the subset $W_{\infty}^{s}(p) \cap \Omega$, which is dense in $\Omega_{c}$, where $\Omega_{c} \subset \Omega$ is the $C$-dense component of $\Omega$ containing $p$. 
Proof. Due to Anosov [2] and Bowen [8], $W^{u}(x) \cap W^{s}(y)$ is dense in $\Omega_{c}$ for any point $x, y \in \Omega_{c}$. It follows that $W_{\infty}^{s}(p) \cap \Omega$ is dense in $\Omega_{c}$, because $W_{\emptyset}^{s}(p) \cap \Omega=\emptyset$.

Corollary 1.1. Suppose $p$ is a boundary point of a codimension one expanding attractor $\Omega$ and $W_{\infty}^{s}(p)$ is a unique component of $W^{s}(p)-p$ which intersects $\Omega$. Then, for any $\delta>0, W_{\infty, \delta}^{s}(p)=W_{\infty}^{s}(p) \cap W_{\delta}^{s}(p)$ intersects $\Omega$. Moreover,

- Given a point $x \in W^{u}(p)-p$, there is a unique arc $(x, y)^{s}$ such that $(x, y)^{s} \cap$ $\Omega=\emptyset$ and $y \in \Omega$.

- Given a point $x \in W^{u}(p)-p$, there is the component $W_{\infty, \delta}^{s}(x)$ of $W_{\delta}^{s}(x)-x$, such that $W_{\infty, \delta}^{s}(x) \cap \Omega \neq \emptyset$ for any $\delta>0$.

Proof. This follows from Lemma1.5 and the theorem on the continuous dependence of invariant manifolds on initial conditions.

Lemma 1.6. A codimension one expanding attractor $\Omega$ (orientable or not) has finitely many boundary points.

Proof. This follows from the local product structure on $\Omega$.

The arc $(x, y)^{s}$ mentioned in Corollary 1.1 (i.e., such that $(x, y)^{s} \cap \Omega=\emptyset$ ) will be denoted by $(x, y)_{\emptyset}^{s}$.

Lemma 1.7. Let $\Omega$ be a codimension one expanding attractor (orientable or not) of an A-diffeomorphism $f: M^{n} \rightarrow M^{n}, n \geq 2$. Suppose $W^{u}(x), x \in \Omega$, does not contain boundary periodic points. Then both components of $W_{\delta}^{s}(x)-x$ intersect $\Omega$ for any $\delta>0$.

Proof. First assume that $W^{u}(x)$ contains a periodic point $x_{0} \in \Omega$. Then $x_{0}$ is an interior periodic point. Therefore both components of $W^{s}\left(x_{0}\right)-x_{0}$ intersect $\Omega$. Hence both components of $W_{\delta}^{s}\left(x_{0}\right)-x_{0}$ intersect $\Omega$ for any $\delta>0$, because $\Omega$ is invariant under $f$. The theorem on the continuous dependence of invariant manifolds on initial conditions implies the result.

If $W^{u}(x)$ does not contain periodic points, then the result follows from Lemma 1.3 because $W^{u}(\Omega)=\Omega$.

Lemma 1.8. Let $\Omega$ be a codimension one $C$-dense expanding attractor (orientable or not) of an A-diffeomorphism $f: M^{n} \rightarrow M^{n}, n \geq 2$. Suppose that $W^{s}(x), x \in \Omega$, does not contain boundary periodic points. Then both $W^{s+}(x) \cap \Omega$ and $W^{s-}(x) \cap \Omega$ are dense in $\Omega$, where $W^{s \pm}(x)$ are components of $W^{s}(x)-x$.

Proof. Due to the $C$-density of $\Omega, W^{s}(x) \cap \Omega$ is dense in $\Omega$. By Lemma 1.7 and Corollary 1.1, both $W^{s+}(x) \cap \Omega$ and $W^{s-}(x) \cap \Omega$ contain infinitely many points. First we prove that at least one set $W^{s+}(x) \cap \Omega$ or $W^{s-}(x) \cap \Omega$ is dense in $\Omega$. Given any point $z \in \Omega-W^{s}(x)$, there is a sequence $x_{k} \in W^{s}(x) \cap \Omega$ such that $x_{k} \rightarrow z$ as $k \rightarrow \infty$. To be definite, assume that all points $x_{k} \in W^{s+}(x)$. Take any point $y \in \Omega$ and any $\eta$-neighborhood $U_{\eta}(y)$ of $y, \eta>0$. Since $\Omega$ is $C$-dense, it follows that there is the arc $\left[z, z_{\eta}\right]^{s}$ with $z_{\eta} \in U_{\eta}(y)$. By the theorem on continuous dependence of stable invariant manifolds on initial conditions, there is $\delta>0$ such that every stable manifold $W^{s}\left(x_{k}\right)$ passing through a $\delta$-neighborhood $U_{\delta}(z)$ of $z$ must intersect $U_{\eta}(y)$ at a point $y_{k}$ with $\left[x_{k}, y_{k}\right]^{s} \subset U_{\delta}\left(\left[z, z_{\eta}\right]^{s}\right)$, where $U_{\delta}\left(\left[z, z_{\eta}\right]^{s}\right)$ is the $\delta$-neighborhood of the arc $\left[z, z_{\eta}\right]^{s}$. Let us take $\delta>0$ so that $x \notin U_{\delta}\left(\left[z, z_{\eta}\right]^{s}\right)$. Then $x \notin\left[x_{k}, y_{k}\right]^{s}$, and thus $W^{s+}(x) \cap U_{\eta}(y) \neq \emptyset$. Hence, $W^{s+}(x) \cap \Omega$ is dense in $\Omega$. 
It remains to prove that $W^{s-}(x) \cap \Omega$ is dense in $\Omega$. Since $W^{s-}(x) \cap \Omega$ contains infinitely many points and $W^{s}(x)$ does not contain boundary periodic points, it follows that there exists a sequence $x_{k}^{\prime} \in W^{s-}(x) \cap \Omega$ that converges to some point $z^{\prime} \in \Omega$ as $k \rightarrow \infty$. Moreover, due to Lemma 1.7, one can assume that the points $x_{k}^{\prime}$ have no accumulation points in $W^{s-}(x)$ with respect to the interior topology of $W^{s-}(x)$. There are two cases: 1) $\left.z^{\prime} \notin W^{s}(x) ; 2\right) z^{\prime} \in W^{s}(x)$. In the first case, the argument above shows that $W^{s-}(x) \cap \Omega$ is dense in $\Omega$. Let us consider the second case. Denote by $W^{s+}\left(z^{\prime}\right)$ the component of $W^{s}\left(z^{\prime}\right)-z^{\prime}$ that is dense in $\Omega$ and intersects with $W^{s+}(x)$. Since the points $x_{k}^{\prime}$ have no accumulation points in $W^{s-}(x)$, we see that there are finitely many points $x_{1}^{\prime}, \ldots, x_{k_{0}}^{\prime}$ on the arc $\left[z^{\prime}, x\right]^{s}$. As above, we conclude that $W^{s-}(x) \cap \Omega$ is dense in $\Omega$.

Lemma 1.9. Let $\Omega$ be a codimension one expanding attractor (orientable or not) of an A-diffeomorphism $f: M^{n} \rightarrow M^{n}, n \geq 2$. If a point $z$ belongs to $W^{s}(\Omega)-\Omega$, then either $z \in(x, y)_{\emptyset}^{s}$, where $x \in W^{u}(p)$ and $y \in W^{u}(q)$ for some periodic boundary points $p, q \in \Omega$ (maybe $p=q$ ), or $z \in W_{\emptyset}^{s}(p)$ for some periodic boundary point $p \in \Omega$.

Proof. Due to [28], any point whose forward orbit stays near $\Omega$ is asymptotic with a point of $\Omega$. Hence, $z \in W^{s}\left(x_{0}\right)$ for some $x_{0} \in \Omega$. Since $\Omega$ is closed, it follows that either $z \in(x, y)^{s}$ with $x, y \in \Omega$ and $(x, y)^{s} \cap \Omega=\emptyset$, or $z$ belongs to a component of $W^{s}(p)-p(p \in \Omega)$ which does not intersect $\Omega$. In the last case, by Lemmas 1.2 and 1.7, $p$ is a periodic boundary point and $z \in W_{\emptyset}^{s}(p)$. If $z \in(x, y)^{s}$, then, according to Lemma 1.7 $x \in W^{u}(p)$ and $y \in W^{u}(q)$ with periodic boundary points $p, q \in \Omega$.

Let $G \subset M$ be an open domain with the boundary $\partial G=$ clos $G$-int $G$. In studying a neighborhood of basic sets, an important feature is which points of $\partial G$ are accessible from within $G$. A point $x \in \partial G$ is called accessible from within $G$ provided there is an open arc $a$ which is in $G$ and $x$ is an endpoint of $a$. The accessible points form the subset $\delta(G) \subset \partial G$, which is called the accessible boundary of $G$.

Lemma 1.10. Let $\Omega$ be a codimension one expanding attractor (orientable or not) of an A-diffeomorphism $f: M^{n} \rightarrow M^{n}, n \geq 2$. Given any point $x \in \Omega$, in the interior topology of the stable manifold $W^{s}(x)$, the intersection $W^{s}(x) \cap \Omega$ is a Cantor set (i.e., a compact totally disconnected perfect set). Moreover, the accessible points in $W^{s}(x) \cap \Omega$ from within $W^{s}(x)-\Omega$ belong to unstable invariant (codimension one) manifolds of periodic boundary points.

Proof. Since $\Omega$ is a compact set and attractor, $W^{s}(x) \cap \Omega$ is a compact totally disconnected set. Let $\Omega_{c} \subset \Omega$ be the $C$-dense component of $\Omega$ containing $x$. Then $W^{u}(z)$ is dense in $\Omega_{c}$ for any $z \in \Omega_{c}$ [2], [8]. It follows that $W^{s}(x) \cap \Omega=W^{s}(x) \cap \Omega_{c}$ is a perfect set.

Lemma 1.9 implies that accessible points in $W^{s}(x) \cap \Omega$ from within $W^{s}(x)-\Omega$ correspond to unstable invariant manifolds of periodic boundary points of $\Omega$.

An unstable manifold $W^{u}(x) \subset \Omega, x \in \Omega$, is called a boundary unstable manifold of $\Omega$ if $W^{u}(x)$ contains a boundary periodic point.

Corollary 1.2. The accessible boundary $\delta\left(M^{n}-\Omega\right)$ of the domain $M^{n}-\Omega$ is a finite union of boundary unstable (codimension one) manifolds of $\Omega$. 
The boundary unstable manifolds of $\Omega$ split into a finite number of so-called bunches in the following way. The pairwise disjoint unstable manifolds $W^{u}\left(p_{1}\right), \ldots$, $W^{u}\left(p_{k}\right)$ is said to be a $k$-bunch if there are points $x_{i} \in W^{u}\left(p_{i}\right)$ and $\operatorname{arcs}$

$$
\left(x_{i}, y_{i}\right)_{\emptyset}^{s}, \quad y_{i} \in W^{u}\left(p_{i+1}\right), \quad 1 \leq i \leq k, \text { where } p_{k+1}=p_{1}, y_{k} \in W^{u}\left(p_{1}\right),
$$

and there are no $(k+1)$-bunches containing the given one. The boundary periodic points $p_{1}, \ldots, p_{k}$ are called associated.

Let $B$ be a $k$-bunch of $\Omega$ consisting of the boundary unstable manifolds $W^{u}\left(p_{1}\right)$, $\ldots, W^{u}\left(p_{k}\right)$, where $p_{i}(i=1, \ldots, k)$ are boundary periodic points. By Corollary 1.1. given any point $x \in W^{u}\left(p_{1}\right)$ with $x \neq p_{1}$, there is a unique point $y \in W^{u}(q)$ such that $(x, y)^{s}=(x, y)_{\emptyset}^{s}$, where $q \in \Omega$ is a boundary periodic point. By definition, put $\varphi_{1}(x)=y$. Hence, $\varphi_{1}: W^{u}\left(p_{1}\right)-p_{1} \rightarrow \Omega$.

Lemma 1.11. Let $B$ be a $k$-bunch of $\Omega$ consisting of the boundary unstable manifolds $W^{u}\left(p_{1}\right), \ldots, W^{u}\left(p_{k}\right)$, where $p_{i}(i=1, \ldots, k)$ are boundary periodic points. Let $\varphi_{1}: W^{u}\left(p_{1}\right)-p_{1} \rightarrow \Omega$ be the mapping defined above. Then the following statements hold:

- $q$ is one of the points $p_{1}$ or $p_{2}$

- $B$ is either a 1-bunch (here $\left.q=p_{1}\right)$ or a 2-bunch (here $q=p_{2} \neq p_{1}$ ).

- $\varphi_{1}$ is a homeomorphism of $W^{u}\left(p_{1}\right)-p_{1}$ onto $W^{u}\left(p_{j}\right)-p_{j}$, where $j$ is equal to either 1 or 2 .

Proof. By definition of a bunch, $\varphi_{1}\left(x_{1}\right)=y_{1} \in W^{u}\left(p_{2}\right)$ for some point $x_{1} \in W^{u}\left(p_{1}\right)$. (It is possible that $p_{2}=p_{1}$, i.e., $k=1$.) Due to the theorem on the continuous dependence of invariant manifolds on initial conditions, $\varphi_{1}$ is a continuous map. Denote by $\operatorname{Dom}\left(x_{1}\right) \subset W^{u}\left(p_{1}\right)-p_{1}$ the set of points which are mapped to $W^{u}\left(p_{2}\right)$ via $\varphi_{1}$. Then $\operatorname{Dom}\left(x_{1}\right) \neq \emptyset$. It follows from the local product structure that $\operatorname{Dom}\left(x_{1}\right)$ is an open and closed subset of $W^{u}\left(p_{1}\right)-p_{1}$. Since $W^{u}\left(p_{1}\right)-p_{1}$ is a connected set (recall that $\operatorname{dim} W^{u}\left(p_{1}\right) \geq 2$ ), we see that $\operatorname{Dom}\left(x_{1}\right)=W^{u}\left(p_{1}\right)-p_{1}$. $\varphi_{1}$ is injective because stable invariant manifolds are pairwise disjoint if they do not coincide. Thus, $\varphi_{1}: W^{u}\left(p_{1}\right)-p_{1} \rightarrow W^{u}\left(p_{2}\right)-p_{2}$ is a homeomorphism.

If $p_{2}=p_{1}$, then $B$ is a 1-bunch. Otherwise, $B$ is a 2-bunch.

Corollary 1.3. If $\Omega$ is a codimension one orientable expanding attractor of an A-diffeomorphism $f: M^{n} \rightarrow M^{n}(n \geq 3)$, then every bunch of $\Omega$ is a 2-bunch.

Proof. Suppose the contrary; then, by Lemma 1.11, there is a 1-bunch $B=W^{u}\left(p_{1}\right)$ $\in \Omega$. Hence, $\varphi_{1}(x) \in W^{u}\left(p_{1}\right)$ for any $x \in W^{u}\left(p_{1}\right)-p_{1}$. By Corollary 1.1, the arc $\left[x, \varphi_{1}(x)\right]^{s}$ intersects $W^{u}\left(p_{1}\right)$ with opposite indexes of intersection at the points $x$, $\varphi_{1}(x)$. We get a contradiction to the orientability of $\Omega$.

Corollary 1.4. Suppose $\Omega$ is a codimension one orientable expanding attractor of an A-diffeomorphism $f: M^{n} \rightarrow M^{n}(n \geq 3)$ and $p, q \in \Omega$ are associated periodic points. Then $p$ and $q$ have the same period.

Thus, the number of boundary periodic points of an orientable pseudotame codimension one basic set is even. These boundary periodic points split into pairs of associated points. Obviously, every boundary unstable manifold belongs to a unique bunch. Note that on every closed surface (two-dimensional manifold), given a natural $k$, there are codimension one pseudotame basic sets with $k$-bunches [5]. 


\section{Characteristic SPHERES AND CONNECTING CYlinders}

Throughout this section $\Omega$ is an orientable expanding attractor of codimension one. By orientability, any bunch of $\Omega$ is a 2-bunch (see Corollary [1.3). Let $B_{p q}$ be the bunch consisting of two unstable manifolds $W^{u}(p)$ and $W^{u}(q)$, where the boundary periodic points $p$ and $q$ are associated. By Corollary 1.4 $p$ and $q$ have the same period, denoted by $m=m(p, q)$. Due to Lemma 1.11 given any point $x \in W^{u}(p)$, there is a unique point $y \in W^{u}(q)$ such that $(x, y)^{s}=(x, y)_{\emptyset}^{s}$, and vice versa. Let the map

$$
\varphi_{p q}:\left(W^{u}(p)-p\right) \cup\left(W^{u}(q)-q\right) \rightarrow\left(W^{u}(p)-p\right) \cup\left(W^{u}(q)-q\right)
$$

be given by $\varphi_{p q}(x)=y$ whenever $(x, y)^{s}=(x, y)_{\emptyset}^{s}$. According to the theorem on the continuous dependence of stable and unstable manifolds on initial conditions, $\varphi_{p q}$ is a homeomorphism. It follows from $f(\Omega)=\Omega$ that

$$
\left.f^{m j} \circ \varphi_{p q}\right|_{\left(W^{u}(p)-p\right) \cup\left(W^{u}(q)-q\right)}=\left.\varphi_{p q} \circ f^{m j}\right|_{\left(W^{u}(p)-p\right) \cup\left(W^{u}(q)-q\right)} \text { for any } j \in \mathbb{Z} \text {. }
$$

The restriction $\left.f^{m}\right|_{W^{u}(p)}$ has the sole hyperbolic repelling fixed point $p$. Therefore there is the closed $C^{1}$ embedded $(n-1)$-ball $D_{p} \subset W^{u}(p)$ bounded by the $C^{1}$ embedded (in $\left.W^{u}(p)\right)(n-2)$-sphere $S_{p}^{n-2}=\partial D_{p}$ such that the following condition holds:

$$
\text { - } p \in \operatorname{int}\left(D_{p}\right)=D_{p}-\partial D_{p}, \quad D_{p} \subset \text { int }\left(f^{m}\left(D_{p}\right)\right) \text {. }
$$

Hence, $S_{p}^{n-2} \cap f^{m}\left(S_{p}^{n-2}\right)=\emptyset$ and $S_{p}^{n-2}$ is inside of $f^{m}\left(S_{p}^{n-2}\right)$. Since $S_{p}^{n-2}$ and $f^{m}\left(S_{p}^{n-2}\right)$ are $C^{1}$ embedded spheres, it follows that they satisfy one more condition (see, e.g., 52]):

- $(n-2)$-spheres $S_{p}^{n-2}$ and $f^{m}\left(S_{p}^{n-2}\right)$ bound a closed $(n-1)$-annulus, call it $A_{p}$, which is homeomorphic to $\Sigma^{n-2} \times[0,1]$.

Lemma 2.1. $\varphi_{p q}$ is extended to the homeomorphism

$$
\varphi_{p q}: W^{u}(p) \cup W^{u}(q) \rightarrow W^{u}(p) \cup W^{u}(q),
$$

if we put $\varphi_{p q}(p)=q$ and $\varphi_{p q}(q)=p$.

Proof. Since $\left.\varphi_{p q}\right|_{\left(W^{u}(p)-p\right)}$ is a homeomorphism, we see that $\varphi_{p q}\left(S_{p}^{n-2}\right)$ is the tamely embedded (in $\left.W^{u}(q)\right)(n-2)$ )-sphere $S_{q}^{n-2}$. From the generalized Schoenflies theorem [9] it follows that $S_{q}^{n-2}$ bounds in $W^{u}(q)$ the closed $(n-1)$-ball, say $D_{q} \subset W^{u}(q)$.

The point $q$ is a unique repelling fixed point of the restriction $\left.f^{m}\right|_{W^{u}(q)}$. Therefore there is $j_{0} \in \mathbb{N}$ such that $D_{q} \subset f^{j_{0} m}\left(D_{q}\right)$. From (1) it follows that

$$
\varphi_{p q} \circ f^{m j_{0}}\left(S_{p}^{n-2}\right)=f^{m j_{0}} \circ \varphi_{p q}\left(S_{p}^{n-2}\right)=f^{j_{0} m}\left(S_{q}^{n-2}\right) \subset W^{u}(q)-D_{q} .
$$

Note that

$$
D_{p} \subset \operatorname{int}\left(f^{m}\left(D_{p}\right)\right) \subset \operatorname{int}\left(f^{m j_{0}}\left(D_{p}\right)\right) .
$$

Hence, $\varphi_{p q}\left(D_{p}-p\right)=D_{q}-q$, because $f^{m j_{0}}\left(S_{p}^{n-2}\right) \subset W^{u}(p)-D_{p}$. As a consequence,

$$
\varphi_{p q} \circ f^{j m}\left(D_{p}-p\right)=f^{j m} \circ \varphi_{p q}\left(D_{p}-p\right)=f^{j m}\left(D_{q}-q\right) .
$$

In the same way one can prove that

$$
\varphi_{p q} \circ f^{j m}\left(D_{q}-q\right)=f^{j m} \circ \varphi_{p q}\left(D_{q}-q\right)=f^{j m}\left(D_{p}-p\right) .
$$

This implies the result, because $\bigcap_{j \leq 0} f^{j m}\left(D_{p}\right)=p$ and $\bigcap_{j \leq 0} f^{j m}\left(D_{q}\right)=q$. 
Corollary 2.1. The following conditions hold:

- $q \in \operatorname{int}\left(D_{q}\right), \quad D_{q} \subset$ int $\left(f^{m}\left(D_{q}\right)\right)$.

- $(n-2)$-spheres $S_{q}^{n-2}=\varphi_{p q}\left(S_{p}^{n-2}\right)$ and $f^{m}\left(S_{q}^{n-2}\right)$ bound the closed $(n-1)$ annulus $A_{q}=\varphi_{p q}\left(A_{p}\right)$, which is homeomorphic to $\Sigma^{n-2} \times[0,1]$.

Moreover,

$$
\left.f^{m j} \circ \varphi_{p q}\right|_{W^{u}(p) \cup W^{u}(q)}=\left.\varphi_{p q} \circ f^{m j}\right|_{W^{u}(p) \cup W^{u}(q)} \text { for any } j \in \mathbb{Z} .
$$

Suppose that points $x, z$ belong to a stable manifold of some point of $\Omega$. Denote by $\lambda(x, z)$ the length of the arc $(x, z)^{s}$. Actually, the following lemma is a consequence of a local product structure.

Lemma 2.2. The function $\lambda(x, z)$ varies continuously with respect to both $x$ and $z$.

Proof. There exists $j_{0} \in \mathbb{N}$ such that the $\operatorname{arc} f^{j_{0}}(x, z)^{s}=\left(f^{j_{0}}(x), f^{j_{0}}(y)\right)^{s}$ belongs to a neighborhood of $\Omega$ with a local product structure [28]. As a consequence, there is a tube neighborhood $U\left((x, z)^{s}\right)$ of $(x, z)^{s}$ that is homeomorphic by a Hölder homeomorphism to the product of $\mathbb{B}^{n-1}$ and $(0,1)$ so that the arcs of stable manifolds correspond to the segments $b \times(0,1), b \in \mathbb{B}^{n-1}[27$. This implies that $\lambda(\cdot, \cdot)$ varies continuously with respect to the both variables $x$ and $z$.

Lemma 2.3. The set $C_{p q}=\bigcup_{x \in S_{p}^{n-2}}\left(x, \varphi_{p q}(x)\right)_{\emptyset}^{s}$ is homeomorphic to the open $(n-1)$-cylinder $\Sigma^{n-2} \times(0,1)$.

Proof. Let $\nu: S_{p}^{n-2} \rightarrow \Sigma^{n-2}$ be an arbitrary homeomorphism. Let $\lambda(x, z)$ be the length of the $\operatorname{arc}(x, z)^{s} \subset(x, y)_{\emptyset}^{s}, x \in S_{p}^{n-2}$. Due to Lemma 2.2 $\lambda(x, z)$ varies continuously with respect to both $x$ and $z$. Moreover, $\lambda\left(x, \varphi_{p q}(x)\right) \geq \alpha$ for all $x \in S_{p}^{n-2}$ and some $\alpha>0$, since $S_{p}^{n-2}$ is a compact set. Define the map $\nu_{p q}: C_{p q} \rightarrow \Sigma^{n-2} \times(0,1)$ as

$$
\nu_{p q}(z)=\left(\nu(x), \frac{\lambda(x, z)}{\lambda\left(x, \varphi_{p q}(x)\right)}\right), \text { where } z \in\left(x, \varphi_{p q}(x)\right)_{\emptyset}^{s} .
$$

This map is one-to-one, because the stable manifolds of $\Omega$ are pairwise disjoint. Since $\lambda(x, z)$ is continuous and $S_{p}^{n-2} \cap S_{q}^{n-2}=\emptyset, \nu_{p q}$ is a homeomorphism.

Corollary 2.2. The set

$$
\text { clos } C_{p q}=C_{p q} \cup S_{p}^{n-2} \cup S_{q}^{n-2}=\bigcup_{x \in S_{p}^{n-2}}\left[x, \varphi_{p q}(x)\right]_{\emptyset}^{s}
$$

is homeomorphic to the closed $(n-1)$-cylinder $\Sigma^{n-2} \times[0,1]$.

The set $C_{p q}=\bigcup_{x \in \partial D_{p}}\left(x, \varphi_{p q}(x)\right)_{\emptyset}^{s}$ is called a connecting cylinder corresponding to the bunch $B_{p q}=W^{u}(p) \cup W^{u}(q)$.

Lemma 2.4. The set $S_{p q}=D_{p} \cup D_{q} \cup C_{p q}$ is homeomorphic to the $(n-1)$-sphere $\Sigma^{n-1}$.

Proof. By construction, $D_{p} \cap D_{q}=\emptyset, D_{p} \cap C_{p q}=\emptyset$, and $D_{q} \cap C_{p q}=\emptyset$. If we combine this with Corollary 2.2 we get the result. 
$S_{p q}=D_{p} \cup D_{q} \cup C_{p q}$ is called a characteristic sphere corresponding to the bunch $B_{p q}=W^{u}(p) \cup W^{u}(q)$. Note that neither the characteristic sphere nor the connecting cylinder corresponding to the bunch $B_{p q}$ is unique. Actually, they depend on the choice of the $(n-1)$-ball $D_{p}$.

Recall that $(n-2)$-spheres $S_{p}^{n-2}$ and $f^{m}\left(S_{p}^{n-2}\right)$ bound in $W^{u}(p)$ the closed $(n-1)$-annulus $A_{p}$, which is homeomorphic to $\Sigma^{n-2} \times[0,1]$. Due to the properties (3)

$$
W^{u}(p)-p=\bigcup_{j \in \mathbb{Z}} f^{j m}\left(A_{p}\right), \quad \text { int }\left(f^{j m}\left(A_{p}\right)\right) \cap i n t\left(f^{k m}\left(A_{p}\right)\right)=\emptyset \quad(j \neq k),
$$

$A_{p}$ is called a fundamental annulus of $\left.f^{m}\right|_{W^{u}(p)}$. It follows from Corollary 2.1 that $A_{q}=\varphi\left(A_{p}\right)$ is a fundamental annulus of $\left.f^{m}\right|_{W^{u}(q)}$, i.e.,

$$
W^{u}(q)-q=\bigcup_{j \in \mathbb{Z}} f^{j m}\left(A_{q}\right), \quad \text { int }\left(f^{l m}\left(A_{q}\right)\right) \cap \operatorname{int}\left(f^{k m}\left(A_{q}\right)\right)=\emptyset \quad(l \neq k) .
$$

By definition, put

$$
A_{p q} \stackrel{\text { def }}{=} \bigcup_{x \in A_{p}}[x, y]_{\emptyset}^{s}=\bigcup_{x \in A_{p}}\left[x, \varphi_{p q}(x)\right]_{\emptyset}^{s}=\bigcup_{x \in A_{q}}\left[x, \varphi_{p q}(x)\right]_{\emptyset}^{s} .
$$

Lemma 2.5. There is a homeomorphism $h_{p q}: A_{p q} \rightarrow A_{p} \times[0,1]$ such that

$$
h_{p q}\left([x, y]_{\emptyset}^{s}\right)=\{x\} \times[0,1] \text { for any } x \in A_{p} .
$$

Proof. Let $\lambda(x, z)$ be the length of the closed arc $[x, z]^{s} \subset(x, y)_{\emptyset}^{s}$. Define $h_{p q}$ as

$$
h_{p q}(z)=\left(x, \frac{\lambda(x, z)}{\lambda\left(x, \varphi_{p q}(x)\right)}\right), \quad \text { where } z \in\left[x, \varphi_{p q}(x)\right]^{s} \subset A_{p q} .
$$

Since the $\operatorname{arcs}\left[x, \varphi_{p q}(x)\right]_{\emptyset}^{s},\left[x^{\prime}, \varphi_{p q}\left(x^{\prime}\right)\right]_{\emptyset}^{s}$ with $x \neq x^{\prime}$ are pairwise disjoint, we see that $h_{p q}$ is one-to-one. Due to Lemma [2.2 $h_{p q}$ is a homeomorphism.

As a consequence, $A_{p q}$ is homeomorphic to the $n$-annulus $\Sigma^{n-2} \times[0,1]^{2}$. By definition, put

$$
D_{p q}\left(S_{p q}\right) \stackrel{\text { def }}{=} D_{p q}=\bigcup_{j \geq 0} f^{j m}\left(A_{p q}\right) .
$$

It follows from (3) and (44) that

$$
f^{j m}\left(C_{p q}\right) \subset D_{p q}, \quad j \geq 0 .
$$

Lemma 2.6. We have $D_{p q}=\bigcup_{x \in W^{u}(p)-i n t D_{p}}\left[x, \varphi_{p q}(x)\right]_{\emptyset}^{s}$.

Proof. It follows from

$$
A_{p q}=\bigcup_{x \in A_{p}}[x, y]_{\emptyset}^{s}, \text { and } W^{u}(p)-i n t D_{p}=\bigcup_{j \geq 0} f^{j m}\left(A_{p}\right)
$$

that

$$
D_{p q} \subset \bigcup_{x \in W^{u}(p)-i n t}\left[x, \varphi_{p}(x)\right]_{\emptyset}^{s} .
$$

Take $\left[x^{\prime}, \varphi_{p q}\left(x^{\prime}\right)\right]_{\emptyset}^{s}$ with $x^{\prime} \in W^{u}(p)-$ int $D_{p}$. Due to (3), $x^{\prime}=f^{j m}(x)$ for some $j \geq 0$ and $x \in W^{u}(p)-$ int $D_{p}$. Thus,

$$
\bigcup_{x \in W^{u}(p)-i n t}\left[x, \varphi_{p q}(x)\right]_{\emptyset}^{s} \subset D_{p q} .
$$


Denote by $\pi_{p}: D_{p q} \rightarrow W^{u}(p)$ - int $D_{p}$ the projection that takes each point $a \in D_{p q}$ to $x$ whenever $a \in[x, y]_{\emptyset}^{s} \subset D_{p q}$. According to Lemma 2.6 the map $\pi_{p}$ is well-defined because the $\operatorname{arcs}\left[x, \varphi_{p q}(x)\right]_{\emptyset}^{s},\left[x^{\prime}, \varphi_{p q}\left(x^{\prime}\right)\right]_{\emptyset}^{s}$ are pairwise disjoint whenever $x \neq x^{\prime}$.

Lemma 2.7. The triple $\left(D_{p q}, W^{u}(p)\right.$ - int $\left.D_{p}, \pi_{p}\right)$ is a trivial fiber bundle with fiber the interval $[0 ; 1]$.

Proof. Note that due to Lemma 2.5 the triple $\left(D_{p q}, W^{u}(p)-\right.$ int $\left.D_{p}, \pi_{p}\right)$ is a fiber bundle with fiber the unit interval $[0 ; 1]$, because $D_{p q}=\bigcup_{j \geq 0} f^{j m}\left(A_{p q}\right)$ and $A_{p q} \cong A_{p} \times[0 ; 1]$ is a fiber bundle with fiber the unit interval $[0 ; 1]$. In order to prove that this fiber bundle is trivial, we have to construct a bundle map $H_{p q}$ : $D_{p q} \rightarrow\left(W^{u}(p)-\right.$ int $\left.D_{p}\right) \times[0,1]$, which is an isomorphism to the product bundle

$$
\left(\left(W^{u}(p)-\text { int } D_{p}\right) \times[0,1], W^{u}(p)-\text { int } D_{p}, p r_{1}\right),
$$

where $p r_{1}:\left(W^{u}(p)-\right.$ int $\left.D_{p}\right) \times[0,1] \rightarrow W^{u}(p)-$ int $D_{p}$ is the projection on the first factor. Define $H_{p q}$ as

$$
H_{p q}(z)=\left(x, \frac{\lambda(x, z)}{\lambda\left(x, \varphi_{p q}(x)\right)}\right), \quad \text { where } z \in\left[x, \varphi_{p q}(x)\right]^{s} \subset D_{p q} .
$$

It immediately follows that $H_{p q}$ takes each $\pi_{p}^{-1}(x)$ to $\{x\} \times[0,1]$, where $x \in W^{u}(p)-$ int $D_{p}$. Thus, $H_{p q}$ is a bundle map. Since the $\operatorname{arcs}\left[x, \varphi_{p q}(x)\right]_{\emptyset}^{s},\left[x^{\prime}, \varphi_{p q}\left(x^{\prime}\right)\right]_{\emptyset}^{s}$ with $x \neq x^{\prime}$ are pairwise disjoint, we see that $H_{p q}$ is one-to-one. Due to Lemma 2.2 $H_{p q}$ is a homeomorphism (here we consider $D_{p q}$ to be endowed with the interior topology).

Corollary 2.3. Each inclusion $S_{p}=\partial D_{p} \subset D_{p q}, S_{q}=\partial D_{q} \subset D_{p q}$ induces the isomorphism

$$
\pi_{n-2}\left(S_{p}\right) \rightarrow \pi_{n-2}\left(D_{p q}\right), \quad \pi_{n-2}\left(S_{q}\right) \rightarrow \pi_{n-2}\left(D_{p q}\right) \cong \mathbb{Z},
$$

respectively. Moreover, both $S_{p}$ and $S_{q}$ are homotopy equivalent to $D_{p q}$.

Proof. Due to Lemma2.7 $W^{u}(p)-$ int $D_{p}$ is a retract of $D_{p q}$. To conclude the proof, it remains to note that $S_{p}$ is a retract of $W^{u}(p)$-int $D_{p}$ because $W^{u}(p) \cong \mathbb{R}^{n-1}$.

Now we will show that the property of characteristic spheres to bound a ball in $M^{n}$ depends on an embedding of $\Omega$ into $M^{n}$ but not on the choice of characteristic spheres. Let $S_{p q}=D_{p} \cup D_{q} \cup C_{p q}$ and $S_{p q}^{\prime}=D_{p}^{\prime} \cup D_{q}^{\prime} \cup C_{p q}^{\prime}$ be characteristic spheres corresponding to the same 2-bunch $B_{p q}$.

Lemma 2.8. The characteristic spheres $S_{p q}, S_{p q}^{\prime}$ are isotopic in $M^{n}$. In particular, $S_{p q}$ bounds a ball in $M^{n}$ iff $S_{p q}^{\prime}$ does.

Proof. Recall that both $S_{p}=\partial D_{p}$ and $S_{p}^{\prime}=\partial D_{p^{\prime}}$ are $C^{1}$ embedded in $W^{u}(p)$ $(n-2)$-spheres (in particular, they are tamely embedded). Therefore there is an isotopy $F_{t}: W^{u}(p) \rightarrow W^{u}(p), 0 \leq t \leq 1$, taking $S_{p}$ to $S_{p}^{\prime}$ [36]. Moreover, one can construct $F_{t}$ so that $F_{t}(p)=p$ for all $t$, since $p$ is inside of the spheres $S_{p}$ and $S_{p}^{\prime}$. Hence, $F_{t}$ is extended to an isotopy of the cylinders $C_{p q}, C_{p q}^{\prime}$ and the spheres $S_{q}=\partial D_{q}, S_{q}^{\prime}=\partial D_{q}^{\prime}$. As a consequence, $S_{p q}$ is isotopic to $S_{p q}^{\prime}$. 


\section{Properties of CHARACTERISTIC SPHERES}

Let $\Omega \subset M^{n}$ be an orientable codimension one expanding attractor of an $A$ diffeomorphism $f: M^{n} \rightarrow M^{n}$, and let $\left\{p_{i}, q_{i}\right\}_{i=1}^{k}$ be pairs of associated points of $\Omega$. Each pair $p_{i}, q_{i}$ of associated points corresponds to the 2-bunch $B_{p_{i} q_{i}}=$ $W^{u}\left(p_{i}\right) \cup W^{u}\left(q_{i}\right)$. Denote by $S_{p_{i} q_{i}}$ the characteristic sphere corresponding to $B_{p_{i} q_{i}}$ and consisting of the connecting $(n-1)$-cylinder $C_{p_{i} q_{i}}$ and the closed $(n-1)$-balls

$$
D_{p_{i}} \subset W^{u}\left(p_{i}\right), D_{q_{i}} \subset W^{u}\left(q_{i}\right) \text {, where } D_{p_{i}} \subset \text { int } f^{m_{i}}\left(D_{p_{i}}\right), D_{q_{i}} \subset \text { int } f^{m_{i}}\left(D_{q_{i}}\right),
$$

and $m_{i}=m\left(p_{i}, q_{i}\right)$ is the (same) period of the points $p_{i}, q_{i}(i=1, \ldots, k)$. The $(n-2)$-spheres $S_{p_{i}}^{n-2}=\partial D_{p_{i}}, f^{m_{i}}\left(S_{p_{i}}^{n-2}\right)$ bound in $W^{u}\left(p_{i}\right)$ a closed $(n-1)$-annulus, say $A_{p_{i}}$, which is homeomorphic to $\Sigma^{n-2} \times[0,1]$. By definition, put

$$
A_{p_{i} q_{i}}=\bigcup_{x \in A_{p_{i}}}\left[x, \varphi_{p_{i} q_{i}}(x)\right]_{\emptyset}^{s} \cong A_{p_{i}} \times[0,1] \cong \Sigma^{n-2} \times[0,1]^{2},
$$

where the homeomorphism

$$
\varphi_{p_{i} q_{i}}=\varphi_{i}: W^{u}\left(p_{i}\right) \cup W^{u}\left(q_{i}\right) \rightarrow W^{u}\left(p_{i}\right) \cup W^{u}\left(q_{i}\right)
$$

satisfies property (2) of Corollary 2.1. Due to Lemma 2.6.

$$
D_{p_{i} q_{i}} \stackrel{\text { def }}{=} \bigcup_{j \geq 0} f^{j m}\left(A_{p_{i} q_{i}}\right)=\bigcup_{x \in W^{u}\left(p_{i}\right)-i n t D_{p_{i}}}\left[x, \varphi_{i}(x)\right]_{\emptyset}^{s} .
$$

Lemma 3.1. Suppose $\Omega$ is a codimension one orientable expanding attractor and $\left\{p_{i}, q_{i}\right\}_{i=1}^{k}$ are pairs of associated boundary periodic points of $\Omega$. Then the following properties hold:

1) The set

$$
D(\Omega) \stackrel{\text { def }}{=} \bigcup_{i=1}^{k} \bigcup_{j \geq 0} f^{j m_{i}}\left(A_{p_{i} q_{i}}\right) \cup \Omega=\bigcup_{i=1}^{k} D_{p_{i} q_{i}} \cup \Omega
$$

is the topological n-submanifold of $M^{n}$ with boundary $\partial D(\Omega)=\bigcup_{i=1}^{k} S_{p_{i} q_{i}}$. In particular, the family of characteristic spheres $\bigcup_{i=1}^{k} S_{p_{i} q_{i}}$ divides $M=$ $M^{n}$. Moreover, any component of $D(\Omega)$ contains a unique $C$-dense component of $\Omega$ and every $C$-dense component of $\Omega$ belongs to a unique component of $D(\Omega)$.

2) If each characteristic sphere $S_{p_{i} q_{i}}$ bounds a closed ball, say $E_{p_{i} q_{i}} \subset M$, then

$$
M=\bigcup_{i=1}^{k} E_{p_{i} q_{i}} \cup W^{s}(\Omega)=\bigcup_{i=1}^{k} \bigcup_{j \geq 0} f^{j m_{i}}\left(E_{p_{i} q_{i}}\right) \cup \Omega .
$$

Moreover, $\Omega$ is $C$-dense.

Proof. Taking an iteration of $f$, if necessary, we can assume without loss of generality that all periodic boundary points $\left\{p_{i}, q_{i}\right\}_{i=1}^{k}$ are fixed. Let us show that the first assertion follows from the second one. Cutting $M$ along the characteristic spheres, we get finitely many topological $n$-manifolds $M_{1}, \ldots, M_{l}$. Suppose $M_{1}$ contains boundary unstable manifolds of some boundary fixed points of $\Omega$, and let $\Omega_{1} \subset M_{1} \cap \Omega$ be the part of $\Omega$ that belongs to $M_{1}$ and consists of whole unstable manifolds, i.e., if $W^{u} \cap \Omega_{1} \neq \emptyset$, then $W^{u} \subset M_{1}$.

Let $p$ be a boundary fixed point and $W^{u}(p) \subset M_{1}$. By construction of the characteristic sphere $S_{p q},\left(W^{u}(q)-D_{q}\right) \cap M_{1} \neq \emptyset$, where $q$ is the boundary fixed point 
associated with $p$. Further, $W^{u}(q)-D_{q}$ does not intersect connecting cylinders of $\Omega$, and thus $W^{u}(q)-D_{q}$ can't intersect the boundary $\partial M_{1}$ of $M_{1}$. Therefore, $W^{u}(q) \subset M_{1}$. Thus, $\Omega_{1}$ contains a boundary unstable manifold $W^{u}(p)$ if and only if $\Omega_{1}$ contains the boundary unstable manifold $W^{u}(q)$, where $p, q$ are associated points. Renumbering associated points of $\Omega$, we can assume that the associated points $p_{1}, q_{1}, \ldots, p_{k_{1}}, q_{k_{1}}$ and their unstable manifolds $W^{u}\left(p_{1}\right), W^{u}\left(q_{1}\right)$, $\ldots, W^{u}\left(p_{k_{1}}\right), W^{u}\left(q_{k_{1}}\right)$ are in $M_{1}$. By construction, $\partial M_{1}$ contains the $(n-1)$ spheres $S_{p_{1} q_{1}}, \ldots, S_{p_{k_{1}} q_{k_{1}}}$.

Take any unstable manifold $W^{u}(y) \subset \Omega$ which is different from $W^{u}\left(p_{i}\right), W^{u}\left(q_{i}\right)$, $1 \leq i \leq k_{1}$, and intersects $\partial M_{1}$. Since any unstable manifold $W^{u} \subset \Omega$ does not intersect the (open) connecting cylinders of $\Omega$, we see that the intersection $W^{u}(y) \cap M_{1}$ is the $(n-1)$-ball $D_{r} \subset \Omega$, where $r \in \Omega$ is the boundary fixed point different from $p_{i}, q_{i}, 1 \leq i \leq k_{1}$. The argument shows that if $W^{u}(x) \subset \Omega$ is the unstable manifold through $x \in$ int $M_{1}$, then $W^{u}(x) \subset M_{1}$. Moreover, $W^{u}(x)$ is in int $M_{1}$. Thus, $\Omega_{1}$ is the union of the unstable manifolds $W^{u}\left(p_{1}\right), W^{u}\left(q_{1}\right)$, $\ldots, W^{u}\left(p_{k_{1}}\right), W^{u}\left(q_{k_{1}}\right)$ and the unstable manifolds from $\Omega$ that belongs to int $M_{1}$.

Now let us consider $M_{1}$ to be a subset of $M^{n}$. It follows from (3), (44), and (5) that the set

$$
\hat{M}_{1}^{+} \stackrel{\text { def }}{=} \operatorname{clos}\left(f\left(M_{1}\right)-M_{1}\right) \cup \operatorname{clos}\left(M_{1}-f\left(M_{1}\right)\right)
$$

is a union of annuli of type $A_{p q}$ attached to the boundary $\partial M_{1}$. Hence, $M_{1}$ is a retract of both $\hat{M}_{1}^{+} \cup M_{1}$ and $M_{1}-\hat{M}_{1}^{+}$. As a consequence, given any $W^{u}(x) \subset$ int $M_{1}$ with $x \in \Omega_{1}$, there is a path $\omega$ in $M_{1}-\hat{M}_{1}^{+}$connecting the points $x$ and $p_{1}$, because $M_{1}$ is arcwise connected. It follows that $f^{-1}(x) \in M_{1}$, since $f^{-1}\left(p_{1}\right)=p_{1}$. Hence, $f^{-1}\left(W^{u}(x)\right) \subset M_{1}$. Similarly, considering the set

$$
\hat{M}_{1}^{-} \stackrel{\text { def }}{=} \operatorname{clos}\left(f^{-1}\left(M_{1}\right)-M_{1}\right) \cup \operatorname{clos}\left(M_{1}-f^{-1}\left(M_{1}\right)\right),
$$

one can prove that $f\left(W^{u}(x)\right) \subset M_{1}$. Since $\Omega_{1}$ is a union of unstable manifolds, $f^{ \pm 1}\left(\Omega_{1}\right)=\Omega_{1}$, i.e., $\Omega_{1}$ is invariant under $f$. The argument above shows that any basic set $\Lambda \subset M_{1}$ different from $\Omega$ is invariant under $f, f(\Lambda)=\Lambda$, because $\hat{M}_{1}^{-} \cup \hat{M}_{1}^{+} \subset W^{s}(\Omega)$, and hence $\left(\hat{M}_{1}^{-} \cup \hat{M}_{1}^{+}\right) \cap \Lambda=\emptyset$.

The boundary $\partial M_{1}$ of $M_{1}$ consists of $k_{2} \geq k_{1}(n-1)$-spheres. Attaching $n$ balls $B_{1}, \ldots, B_{k_{2}}$ to $M_{1}$ along each $(n-1)$-sphere of $\partial M_{1}$, we obtain the closed $n$-manifold $M_{1}^{\prime}$. Since

$$
\hat{M}_{1}^{-} \cup \hat{M}_{1}^{+}=\bigcup_{i=1}^{k_{1}} f\left(A_{p_{i} q_{i}}\right) \bigcup_{i=k_{1}+1}^{k_{2}} f\left(A_{p_{i} q_{i}}\right),
$$

$\left.f\right|_{M_{1}}$ can be extended to the $A$-diffeomorphism $f_{1}: M_{1}^{\prime} \rightarrow M_{1}^{\prime}$ so that $f_{1}$ has a unique repelling or attracting fixed point $O_{i}$ inside each attached $n$-ball $B_{i}(1 \leq$ $\left.i \leq k_{2}\right)$. To be precise, the point $O_{i}$ is repelling for $1 \leq i \leq k_{1}$ and attracting for $k_{1}+1 \leq i \leq k_{2}$. The set $\Omega_{1} \subset M_{1}$ becomes the invariant set $\Omega^{\prime} \subset M_{1}^{\prime}$ of $f_{1}$ (i.e., $\Omega^{\prime}$ coincides with $\Omega_{1}$ as a set). Moreover, constructing $f_{1}$ carefully, one can get $\Omega^{\prime}$ to be a hyperbolic attractor, because

$$
\Omega_{1} \cap \partial M_{1}=\bigcup_{i=1}^{k_{1}}\left(D_{p_{i}} \cup D_{p_{i}}\right) .
$$

Since $\Omega^{\prime}$ is homeomorphic locally to the product of $\mathbb{R}^{n-1}$ and a Cantor set, $\Omega^{\prime}$ is a codimension one orientable expanding attractor. 
By construction, any characteristic sphere of $\Omega^{\prime}$ bounds a ball. Due to the second assertion,

$$
M_{1}^{\prime}=\bigcup_{\nu=1}^{k_{2}} \bigcup_{j \geq 0} f^{j}\left(E_{p_{\nu} q_{\nu}}\right) \cup \Omega^{\prime}=\bigcup_{\nu=1}^{k_{2}} E_{p_{\nu} q_{\nu}} \cup W^{s}\left(\Omega^{\prime}\right)
$$

and $\Omega^{\prime}$ is a $C$-dense basic set. Since

$$
\bigcup_{\nu=1}^{k_{2}} S_{p_{\nu} q_{\nu}}=\bigcup_{\nu=1}^{k_{2}} \partial E_{p_{\nu} q_{\nu}} \subset W^{s}\left(\Omega^{\prime}\right)
$$

we have $k_{2}=k_{1}$, as otherwise some connecting cylinders would belong to both $W^{s}\left(\Omega^{\prime}\right)$ and the stable manifold of the corresponding attracting fixed point, which is impossible.

Denote $A_{p_{\nu} q_{\nu}}=\operatorname{clos}\left(f\left(E_{p_{\nu} q_{\nu}}\right)-E_{p_{\nu} q_{\nu}}\right), 1 \leq \nu \leq k_{1}$. The argument above shows that

$$
M_{1}=\bigcup_{\nu=1}^{k_{1}} \bigcup_{j \geq 0} f^{j}\left(A_{p_{\nu} q_{\nu}}\right) \cup \Omega^{\prime}
$$

is the topological $n$-submanifold of $M^{n}$ with the boundary $\partial D\left(\Omega^{\prime}\right)=\bigcup_{i=1}^{k_{1}} S_{p_{i} q_{i}}$. Similar reasoning applies to the remaining $n$-manifolds $M_{i}$ containing at least one whole boundary unstable manifold of $\Omega$. Note that if int $M_{i}(1 \leq i \leq l)$ contains an unstable manifold $W^{u} \subset \Omega$ that belongs to a $C$-dense component $\Omega_{c}$ of $\Omega$, then any boundary unstable manifold from $\Omega_{c}$ is in $M_{i}$ because $W^{u}$ is dense in $\Omega_{c}$ [2], 8]. As a consequence, $\Omega_{c, i}=\Omega_{c} \subset M_{i}$, where $\Omega_{c, i}=M_{i} \cap \Omega$.

What we have proved is that a component $M_{i}(1 \leq i \leq l)$ contains a unique $C$-dense component $\Omega_{c, i}$ of $\Omega$ whenever $M_{i}$ contains at least one unstable manifold of $\Omega$. Obviously, a union of such components $M_{i}$ forms the set $D(\Omega)$, which splits into pieces of the kind $M_{i}=\bigcup_{\nu=1} \bigcup_{j \geq 0} f^{j}\left(A_{p_{\nu} q_{\nu}}\right) \cup \Omega_{c, i}$. Thus the second assertion implies the first one.

To conclude the proof, it remains to prove the second assertion. We begin with the equality

$$
\bigcup_{i=1}^{k_{1}} E_{p_{i} q_{i}} \cup W^{s}\left(\Omega_{c}\right)=\bigcup_{i=1}^{k_{1}} \bigcup_{j \geq 0} f^{j}\left(E_{p_{i} q_{i}}\right) \cup \Omega_{c} \stackrel{\text { def }}{=} M_{0},
$$

where $\Omega_{c}$ is some $C$-dense component of $\Omega$. Here we suppose that the associated points $\left\{p_{i}, q_{i}\right\}_{i=1}^{k_{1}}, k_{1} \leq k$, belong to $\Omega_{c}$. It follows from (3) and (4) that

$$
A_{p_{i} q_{i}}=\operatorname{clos}\left(f\left(E_{p_{i} q_{i}}\right)-E_{p_{i} q_{i}}\right) \subset D_{p_{i} q_{i}} .
$$

By Lemma 2.6. $D_{p_{i} q_{i}} \subset W^{s}\left(\Omega_{c}\right)$ for $i \leq k_{1}$. Hence,

$$
\bigcup_{i=1}^{k_{1}} \bigcup_{j \geq 0} f^{j}\left(E_{p_{i} q_{i}}\right) \cup \Omega \subset \bigcup_{i=1}^{k_{1}} E_{p_{i} q_{i}} \cup W^{s}\left(\Omega_{c}\right) .
$$

To conclude the inverse inclusion, take a point $z \in W^{s}\left(\Omega_{c}\right)-\Omega_{c}$. By Lemma 1.9 $z$ belongs either to some $\operatorname{arc}(x, y)_{\emptyset}^{s}$ with $x \in W^{u}\left(p_{i}\right)$ and $y \in W^{u}\left(q_{i}\right)$, or $z \in W_{\emptyset}^{s}\left(p_{i}\right)$ for some periodic boundary point $p_{i} \in \Omega_{c}\left(i \leq k_{1}\right)$. In the last case, $z \in E_{p_{i} q_{i}}$. In the case of $z \in(x, y)_{\emptyset}^{s}$, we have to consider two possibilities. If $x \in D_{p_{i}}$, then 
$z \in E_{p_{i} q_{i}}$. If $x \notin D_{p_{i}}$, then $z \in D_{p_{i} q_{i}}$. Thus in any case, $z \in \bigcup_{j \geq 0} f^{j}\left(E_{p_{i} q_{i}}\right)$. Hence,

$$
W^{s}\left(\Omega_{c}\right)-\Omega_{c} \subset \bigcup_{i=1}^{k_{1}} \bigcup_{j \geq 0} f^{j}\left(E_{p_{i} q_{i}}\right) .
$$

Let us show that the set $M_{0}$ is closed and open. Obviously, $W^{s}\left(\Omega_{c}\right)$ is open. Any characteristic sphere $S_{p_{i} q_{i}}$ belongs to $W^{s}\left(\Omega_{c}\right)$, because $\Omega_{c} \subset W^{s}\left(\Omega_{c}\right)$. Hence, $M_{0}=\bigcup_{i=1}^{k_{1}} E_{p_{i} q_{i}} \cup W^{s}\left(\Omega_{c}\right)$ is open.

Take a point $z_{0} \in \operatorname{clos} M_{0}$. Then there is a sequence of points

$$
z_{s} \in \bigcup_{i=1}^{k_{1}} \bigcup_{j \geq 0} f^{j}\left(E_{p_{i} q_{i}}\right) \cup \Omega_{c}
$$

that approach $z_{0}$ as $s \rightarrow \infty$. If there are infinitely many points $z_{s}$ from $\Omega_{c}$, then $z_{0} \in \Omega_{c} \subset M_{0}$, because $\Omega_{c}$ is closed. Therefore we can assume that not all points $z_{s}$ are in $\Omega_{c}$. Then, by Lemma 1.9, $z_{s} \in \bigcup_{j \geq 0} f^{j}\left(E_{p_{i} q_{i}}\right)-\Omega_{c}$ for some $1 \leq i \leq k_{1}$. Hence, $z_{s} \in f^{j_{s}}\left(E_{p_{i} q_{i}}\right)$ for some sequence of natural $j_{s}$. If the sequence $j_{s}$ is bounded, then $z_{0}$ belongs to a finite union of $n$-balls of the type $f^{l m}\left(E_{p_{i} q_{i}}\right)$, and thus, $z_{0} \in M_{0}$. If the sequence $j_{s}$ is unbounded, then $z_{s} \in\left(x_{s}, y_{s}\right)_{\emptyset}^{s} \subset f^{j_{s}}\left(E_{p_{i} q_{i}}\right)$. Due to Lemma 1.10, $\lambda\left(x_{s}, y_{s}\right)_{\emptyset}^{s} \rightarrow 0$ as $s \rightarrow \infty$, where $\lambda(\cdot, \cdot)$ is the length of the arc $(\cdot, \cdot)$. Hence, $\lim _{s \rightarrow \infty} z_{s}=\lim _{s \rightarrow \infty} x_{s}$. Since $x_{s} \in \Omega_{c}, \lim _{s \rightarrow \infty} x_{s} \in \Omega_{c}$. Therefore, $z_{0}=\lim _{s \rightarrow \infty} z_{s} \in \Omega_{c}$. As a consequence, $M_{0}$ is closed. Thus, $M=M_{0}$ because $M$ is connected.

Let us show that $\Omega=\Omega_{c}$. Suppose not; then $\Omega$ has another $C$-dense component, say $\Omega_{1}$, different from $\Omega_{c}$. Since $M=\bigcup_{i=1}^{k_{1}} E_{p_{i} q_{i}} \cup W^{s}\left(\Omega_{c}\right)$, it follows that $\Omega_{1} \subset$ $\bigcup_{i=1}^{k_{1}} E_{p_{i} q_{i}}$, because stable manifolds are pairwise disjoint. Any unstable manifold $W^{u}(x), x \in \Omega_{1}$, is dense in $\Omega_{1}$ [2], [8]. Hence, $\Omega_{1}$ is a connected set, since $W^{u}(x) \subset$ $\Omega_{1}$. As a consequence, $\Omega_{1}$ belongs to a unique ball of the union $\bigcup_{i=1}^{k_{1}} E_{p_{i} q_{i}}$. To be definite, assume that $\Omega_{1} \subset E_{p_{1} q_{1}}$. Due to [2] and [8], $\Omega_{1}$ is a codimension one expanding attractor of $f^{r}$ for some $r \in \mathbb{N}$. Attaching the $n$-ball $\mathbb{B}^{n}$ to $E_{p_{1} q_{1}}$ along the boundaries $\partial \mathbb{B}^{n}=\Sigma^{n-1} \cong \partial E_{p_{1} q_{1}}$, we obtain the $n$-sphere $\Sigma^{n}$. It follows from (5) that $f^{r}$ maps the connecting cylinder $C_{p_{1} q_{1}}$ inside of $D_{p_{1} q_{1}}$. Hence, $f^{r}$ can be extended to $\Sigma^{n}$ so that the resulting diffeomorphism $\Sigma^{n} \rightarrow \Sigma^{n}$ has a codimension one expanding attractor.

On the other hand, Kollmer 32 proved that a simply connected $n$-manifold $(n \geq$ 3) does not admit a hyperbolic attractor of codimension one (see also [42], where it was proved that a codimension one expanding attractor $\Lambda$ determines a non-zero homology class if both subbundles $E_{\Lambda}^{s}$ and $E_{\Lambda}^{u}$ in the splitting $T_{\Lambda} M=E_{\Lambda}^{s} \oplus E_{\Lambda}^{u}$ are oriented). This contradiction concludes the proof.

Corollary 3.1. Suppose the conditions of Lemma 3.1 hold. Let $\Omega^{\prime} \neq \Omega$ be another basic set. If the unstable manifold $W^{u}\left(z^{\prime}\right)$ of some point $z^{\prime} \in \Omega^{\prime}$ intersects $D_{p_{i} q_{i}}$, then $W^{u}\left(z^{\prime}\right)$ intersects the connecting cylinder $C_{p_{i} q_{i}}$.

Proof. Since stable manifolds are pairwise disjoint, $\Omega^{\prime} \cap D_{p_{i} q_{i}}=\emptyset$. Also, $W^{u}\left(z^{\prime}\right)$ is connected and does not intersect $W^{u}\left(p_{i}\right) \cup W^{u}\left(q_{i}\right)$. Then the proof follows from Lemma 3.1 


\section{Some APplications of The LAMinAtion THEORY}

Let $\bar{M}$ be a universal (simply connected) covering space for $M$, and $\pi: \bar{M} \rightarrow M$ a corresponding cover. Two points $\bar{x}, \bar{y} \in \bar{M}$ are said to be congruent if $\pi(\bar{x})=\pi(\bar{y})$. We will generally identify the fundamental group $\pi_{1}(M)$ with the group of covering transformations for the cover $\pi: \bar{M} \rightarrow M$. Thus, $\bar{x}$ and $\bar{y}$ are congruent if and only if there is $\alpha \in \pi_{1}(M)$ such that $\alpha(\bar{x})=\bar{y}$.

Let $f: M \rightarrow M$ be a diffeomorphism. Since $\bar{M}$ is a universal covering, we see that there is a lift $\bar{f}: \bar{M} \rightarrow \bar{M}$ of $f$, i.e., $\bar{f} \circ \pi=\pi \circ f$. Note that $\bar{f}$ is a diffeomorphism as well. If $f$ is an $A$-diffeomorphism, then $\bar{f}$ has the invariant set $\pi^{-1}(N W(f))$ with a local product structure [12, 27, [48. Let us denote by $w^{u(s)}(\bar{x})$ a lift of $W^{u(s)}(x), x \in N W(f)$, where $\bar{x} \in \bar{M}$ is a lift of $x$. We get

$$
\bar{M}=\bigcup_{\bar{x} \in \pi^{-1}(N W(f))} w^{u}(\bar{x})=\bigcup_{\bar{x} \in \pi^{-1}(N W(f))} w^{s}(\bar{x}),
$$

because $M=\bigcup_{x \in N W(f)} W^{u}(x)=\bigcup_{x \in N W(f)} W^{s}(x)$. Since each invariant manifold of $f$ is an injectively immersed copy of Euclidean space, it follows that each $w^{u(s)}(\bar{x})$ is also an injectively immersed copy of Euclidean space. Moreover, the families

$$
\left\{w^{u}(\bar{x})\right\}_{\bar{x} \in \pi^{-1}(N W(f))} \text { and } \quad\left\{w^{s}(\bar{x})\right\}_{\bar{x} \in \pi^{-1}(N W(f))}
$$

are invariant under $\bar{f}$. Therefore, $w^{u(s)}(\bar{x})$ is said to be the unstable (stable) invariant manifold of $\bar{f}$. Thus, $\bar{M}$ splits into invariant manifolds which are lifts of the invariant manifolds of $f$.

Suppose $f: M \rightarrow M$ is an $A$-diffeomorphism with an orientable expanding attractor or contracting repeller $\Omega$ of codimension one; then $\bar{\Omega}=\pi^{-1}(\Omega)$ is an invariant hyperbolic set of $\bar{f}$ consisting of codimension one submanifolds smoothly immersed in $\bar{M}$ 12], 27]. Locally $\bar{\Omega}$ is the product of $(n-1)$-dimensional Euclidean space $\mathbb{R}^{n-1}$ and a Cantor set, because $\pi$ is a local homeomorphism. Obviously one can define the notions of boundary points (not necessary periodic under $\bar{f}$ ), boundary manifolds, and bunches (as a consequence, also associated boundary points) for $\bar{\Omega}$. It is easily shown that the boundary points of $\bar{\Omega}$ are just the points of the preimage $\pi^{-1}\left(B_{\Omega}\right)$, where $B_{\Omega}$ is the set of the boundary points of $\Omega$.

To be definite, assume that $\Omega$ is an expanding attractor. We keep the notation of section 2 .

Lemma 4.1. Let $B_{p q}=W^{u}(p) \cup W^{u}(q)$ be the bunch of $\Omega$ consisting of the unstable manifolds $W^{u}(p), W^{u}(q)$ of the associated boundary periodic points $p$ and $q$. Suppose $\bar{p}$ is a lift of $p$; then there is a unique lift $\bar{q}$ of $q$ such that $\bar{B}_{\overline{p q}}=w^{u}(\bar{p}) \cup w^{u}(\bar{q}) \in \pi^{-1}\left(B_{p q}\right)$ is a 2-bunch of $\bar{\Omega}$. Moreover, there is the homeomorphism

$$
\bar{\varphi}_{\overline{p q}}:\left(w^{u}(\bar{p})-\bar{p}\right) \cup\left(w^{u}(\bar{q})-\bar{q}\right) \rightarrow\left(w^{u}(\bar{p})-\bar{p}\right) \cup\left(w^{u}(\bar{q})-\bar{q}\right),
$$

which covers $\varphi_{p q}$ and is extended to the homeomorphism

$$
\bar{\varphi}_{\overline{p q}}: w^{u}(\bar{p}) \cup w^{u}(\bar{q}) \rightarrow w^{u}(\bar{p}) \cup w^{u}(\bar{q}),
$$

if we put $\bar{\varphi}_{\overline{p q}}(\bar{p})=\bar{q}$ and $\bar{\varphi}_{\overline{p q}}(\bar{q})=\bar{p}$.

Proof. Take an arbitrary point $\bar{x} \in w^{u}(\bar{p})-\bar{p}$. Since $\pi\left(w^{u}(\bar{p})-\bar{p}\right)=W^{u}(p)-p$, we have $x=\pi(\bar{x}) \in W^{u}(p)-p$. Let $[\bar{x}, \bar{y}]^{s}$ be the lift of $\left[x, \varphi_{p q}(x)\right]_{\emptyset}^{s}$. Obviously, $(\bar{x}, \bar{y})^{s} \cap \bar{\Omega}=\emptyset$ and $\bar{y} \in \bar{\Omega}$. Hence, $(\bar{x}, \bar{y})^{s}=(\bar{x}, \bar{y})_{\emptyset}^{s}$. Recall that $B_{\Omega}$ is the set of 
boundary points of $\Omega$. It follows from the definition of boundary points and Lemma 1.7 that there is a point $\bar{q} \in \pi^{-1}\left(B_{\Omega}\right)$. Due to the path lifting theorem (see, e.g., [54], ch. 1), $\bar{q}$ is a lift of the point $q$. By Lemma 1.11] and Corollary 1.3$] \bar{q} \neq \bar{p}$ and $\bar{q}$ does not depend on the choice of the point $\bar{x} \in w^{u}(\bar{p})-\bar{p}$. As a consequence,

$$
\bar{B}_{\overline{p q}}=w^{u}(\bar{p}) \cup w^{u}(\bar{q}) \in \pi^{-1}\left(B_{p q}\right)
$$

is a 2 -bunch of $\bar{\Omega}$ which is a lift of the 2 -bunch $B_{p q}$.

The argument shows that given any point $\bar{x} \in w^{u}(\bar{p})-\bar{p}$, there is a unique point $\bar{y} \in w^{u}(\bar{q})$ such that $(\bar{x}, \bar{y})^{s}=(\bar{x}, \bar{y})_{\emptyset}^{s}$, and vice versa. By definition, put $\bar{\varphi}_{\overline{p q}}(\bar{x})=\bar{y}$, $\bar{\varphi}_{\overline{p q}}(\bar{y})=\bar{x}$. Due to the path lifting theorem, $\bar{\varphi}_{\overline{p q}}$ is a lift of $\varphi_{p q}$. This implies that $\bar{\varphi}_{\overline{p q}}$ is extended to the homeomorphism $\bar{\varphi}_{\overline{p q}}: w^{u}(\bar{p}) \cup w^{u}(\bar{q}) \rightarrow w^{u}(\bar{p}) \cup w^{u}(\bar{q})$, if we put $\bar{\varphi}_{\overline{p q}}(\bar{p})=\bar{q}$ and $\bar{\varphi}_{\overline{p q}}(\bar{q})=\bar{p}$, because $\pi$ is a local homeomorphism.

Corollary 4.1. Suppose $B_{p q}$ is a bunch of $\Omega$; then $\pi^{-1}\left(B_{p q}\right)$ splits into pairwise disjoint 2-bunches of $\bar{\Omega}$. Moreover, each bunch of $\pi^{-1}\left(B_{p q}\right)$ is a lift of $B_{p q}$.

Proof. It follows from Lemma 4.1 that $\pi^{-1}\left(B_{p q}\right)$ consists of 2 -bunches of $\bar{\Omega}$. These bunches are pairwise disjoint, because no invariant manifold of $\bar{\Omega}$ has congruent points.

Lemma 4.2. Let $B_{p q}=W^{u}(p) \cup W^{u}(q)$ be a bunch of $\Omega$ and $\bar{B}_{\overline{p q}}$ a lift of $B_{p q}$ which is a bunch of $\bar{\Omega}$, where $\pi(\bar{p})=p$ and $\pi(\bar{q})=q$. Suppose $S_{p q}$ is a characteristic sphere corresponding to $B_{p q}$ and consisting of a connecting cylinder $C_{p q}$ and two $(n-1)$-balls $D_{p}, D_{q}$. Then there is the characteristic sphere $\bar{S}_{\overline{p q}}$ corresponding to $\bar{B}_{\overline{p q}}$ and consisting of the connecting cylinder $\bar{C}_{\overline{p q}}$ and two $(n-1)$-balls $\bar{D}_{\bar{p}}, \bar{D}_{\bar{q}}$ such that $\pi\left(\bar{D}_{\bar{p}}\right)=D_{p}, \pi\left(\bar{D}_{\bar{q}}\right)=D_{q}$, and $\pi\left(\bar{C}_{\overline{p q}}\right)=C_{p q}$. Moreover, $\pi^{-1}\left(S_{p q}\right)$ splits into pairwise disjoint characteristic spheres which are in one-to-one correspondence to the bunches of $\pi^{-1}\left(B_{p q}\right)$.

Proof. It follows from the path lifting theorem [54] that there is an $(n-1)$-ball $\bar{D}_{\bar{p}} \subset w^{u}(\bar{p})$ such that $\bar{p} \in \bar{D}_{\bar{p}}$ and $\bar{D}_{\bar{p}}$ is a lift of $D_{p}$. Similarly, there is $(n-1)$-ball $\bar{D}_{\bar{q}} \subset w^{u}(\bar{q})$ such that $\bar{q} \in \bar{D}_{\bar{q}}$ and $\bar{D}_{\bar{p} q}$ is a lift of $D_{q}$. By Lemma 4.1

$$
\bar{\varphi}_{\overline{p q}}\left(\bar{D}_{\bar{p}}\right)=\bar{D}_{\bar{q}}, \quad \bar{\varphi}_{\overline{p q}}\left(\bar{D}_{\bar{q}}\right)=\bar{D}_{\bar{p}} \text {. }
$$

Hence, the set $\bar{C}_{\overline{p q}} \stackrel{\text { def }}{=} \bigcup_{\bar{x} \in \partial \bar{D}_{\bar{p}}}\left(\bar{x}, \bar{\varphi}_{\overline{p q}}(\bar{x})\right)_{\emptyset}^{s}$ is homeomorphic to the open $(n-1)$ cylinder $\Sigma^{n-2} \times(0,1)$, and $\pi\left(\bar{C}_{\overline{p q}}\right)=C_{p q}$. As a consequence, the union

$$
\bar{S}_{\overline{p q}}=\bar{D}_{\bar{p}} \cup \bar{D}_{\bar{q}} \bigcup_{\bar{x} \in \partial \bar{D}_{\bar{p}}}(\bar{x}, \bar{y})_{\emptyset}^{S}
$$

is a characteristic sphere corresponding to $\bar{B} \overline{p q}$.

Since no unstable manifold of $\bar{\Omega}$ has congruent points, we see that the restriction of $\pi$ on each characteristic sphere of $\bar{\Omega}$ is injective. This concludes the proof.

Now we recall the notion of a lamination. Let us fix a natural number $1 \leq d \leq n$ and integers $0 \leq l \leq r \leq \infty$. Suppose $N \subset M$ is a closed set. We say that $\overline{\mathcal{L}}$ is a d-dimensional $C^{r, \bar{l}}$-lamination on $N$, supp $\mathcal{L}=N$, if $N$ is a union of pairwise disjoint injectively immersed $d$-manifolds $L_{\alpha}, N=\bigcup_{\alpha} L_{\alpha}$ (called the leaves), and for each $x \in \operatorname{supp} \mathcal{L}$ there are a neighborhood $U(x) \subset M, x \in U(x)$, and a $C^{l}$ diffeomorphism $\varphi_{x}: U(x) \rightarrow \mathbb{R}^{n}$ such that $\varphi_{x}$ maps every connected component of 
the intersection $U(x) \cap L_{\alpha}$ onto a $d$-dimensional hyperplane

$$
x_{d+1}=c_{1}, \ldots, x_{n}=c_{n-d} \text { for some }\left(c_{1}, \ldots, c_{n-d}\right) \in \mathbb{R}^{n-d} .
$$

The restriction $\left.\varphi_{x}\right|_{U(x) \cap L_{\alpha}}$ is a $C^{r}$-diffeomorphism onto the image $\varphi_{x}\left(U(x) \cap L_{\alpha}\right)$.

Notice that a $C^{0}$-diffeomorphism means a homeomorphism. The pair $\left(U(x), \varphi_{x}\right)$ is called a laminated chart. Given any chart $\left(U(x), \varphi_{x}\right)$ and leaf $L_{\alpha}$, connected components of the intersection $U(x) \cap L_{\alpha}$ are called local leaves. For $d=n-1, \mathcal{L}$ is called a codimension one lamination.

In a sense, the notion of lamination is a weakening of the well-known notion of a foliation. If supp $\mathcal{L}=M$, then a $C^{r, l}$-lamination $\mathcal{L}$ is a $C^{l}$-foliation. Many definitions from foliation theory can be easily generalized to lamination theory (good references for foliation theory are Lawson's paper [33] and Tamura's book [57]). We presuppose some knowledge of foliations. As a consequence of the definition above, we get the following lemma.

Lemma 4.3. Let $\mathcal{L}$ be a d-dimensional $C^{r, l}$-lamination and $L_{\alpha}$ a leaf of $\mathcal{L}$. Suppose $X$ is a compact subset of $L_{\alpha}$. Then there are a neighborhood $U(X) \subset M$ of $X \subset$ $U(X)$ and a $C^{l}$-diffeomorphism $\varphi_{X}: U(X) \rightarrow \mathbb{R}^{n}$ such that, given any leaf $L_{\beta}, \varphi_{X}$ maps every component of the intersection $U(X) \cap L_{\beta}$ (a local leaf in $U(X)$ ) onto a ddimensional hyperplane $x_{d+1}=c_{1}, \ldots, x_{n}=c_{n-d}$ for some $\left(c_{1}, \ldots, c_{n-d}\right) \in \mathbb{R}^{n-d}$.

Proof. There is a finite cover of the compact set $X$ by laminated charts $\left(U\left(x_{i}\right), \varphi_{i}\right)$. Similarly to the proof of Theorem 5.1 of [57], one can construct a finite set of coherent laminated charts covering $X$. This implies the result.

Corollary 4.2. If the conditions of Lemma 4.3 hold, then there are a neighborhood $U(X) \subset M, X \in U(X)$, and d-dimensional $C^{t}$-foliation $\mathcal{F}$ in $U(X)$ such that the local leaves of $\mathcal{L}$ in $U(X)$ are leaves of $\mathcal{F}$.

Lemma 4.4. If $\mathcal{L}$ is a codimension one $C^{r, l}$-lamination $(1 \leq l \leq r)$ and $L_{\alpha}$ is a simply connected leaf of $\mathcal{L}$, then the holonomy group of $L_{\alpha}$ is trivial.

Proof. Take a point $x_{0} \in L_{\alpha}$ and a smooth closed loop $\gamma \subset L_{\alpha}$ at $x_{0}$. We assume that $\gamma$ is endowed with some orientation. Let $l$ be a segment through $x_{0}$ which is transverse to $\mathcal{L}$. Then $\mathcal{L}$ induces the map $G_{\gamma}: l \cap \mathcal{L} \rightarrow l \cap \mathcal{L}$ corresponding to the positive direction on $\gamma$. This map defines the element $g_{\gamma}$ of the holonomy group (the reader is referred to [57] for more background on holonomy groups) of the lamination $\mathcal{L}$.

Since $L_{\alpha}$ is simply connected, $\gamma$ is contractible; and we can assume that $\gamma$ bounds a 2-disk $D \subset L_{\alpha}$ because $L_{\alpha}$ is a smoothly immersed submanifold of codimension one. By Corollary 4.2, there is a neighborhood $U(D)$ of $D$ such that $\mathcal{L}$ is embedded in a $C^{l}$-foliation. Reeb's stability theorem [46] implies that $G_{\gamma}=i d$ (see Theorems 5.7 and 5.12 in [57] as well). Hence, $g_{\gamma}=i d$. This concludes the proof.

Let $\mathcal{L}$ be a codimension one $C^{r, l}$-lamination $(1 \leq l \leq r)$ such that each leaf is endowed with a normal orientation. Suppose $L_{\alpha}$ is a leaf of $\mathcal{L}$, and $T$ is an arc or closed curve endowed with some orientation and transversal to $L_{\alpha}$. We say that $T$ intersects $L_{\alpha}$ oriently if the index of intersection at every point of $L_{\alpha} \cap T$ is the same (either +1 or -1 ). 
Let $f: M \rightarrow M$ be an $A$-diffeomorphism with an orientable expanding attractor $\Omega$. Then the unstable manifolds of points of $\Omega$ form the codimension one $C^{\infty, 1}$ _ lamination $\mathcal{L}(\Omega)$ whenever $\Omega$ is a codimension one attractor [27],

$$
\mathcal{L}(\Omega)=\left\{W^{u}(x)\right\}_{x \in \Omega}, \quad \operatorname{supp} \mathcal{L}(\Omega)=\bigcup_{x \in \Omega} W^{u}(x) .
$$

Hence, $\pi^{-1}(\mathcal{L}(\Omega))=\mathcal{L}(\bar{\Omega})$ is a $C^{\infty, 1}$-lamination on $\bar{M}$ consisting of the unstable manifolds of $\bar{\Omega}$, because $\pi$ is a local diffeomorphism.

Lemma 4.5. Let $T \subset M$ be a closed simple curve which is transversal to the leaves of the lamination $\mathcal{L}(\Omega)$. If $T$ intersects oriently all leaves of $\mathcal{L}(\Omega)$, then $T$ is not homotopic to zero in $M$.

Proof. Assume the contrary. Then there is a smooth immersion $\psi: D^{2} \rightarrow M$ such that $\psi\left(\partial D^{2}\right)=T$, where $D^{2}$ is a closed 2-disk. Since $\psi\left(D^{2}\right)$ is a compact set, we see that there is a finite cover $\left\{U_{i}\right\}_{i=1}^{k}$ of $\psi\left(D^{2}\right)$ with the laminated charts $U_{i}, \psi\left(D^{2}\right) \subset \bigcup_{i=1}^{k} U_{i}$. By Lemma 4.3 supp $\mathcal{L}(\Omega) \cap U_{i}$ can be embedded in a $C^{1}$ foliation, say $\mathcal{F}_{i}$, for every $1 \leq i \leq k$. Now we can use an argument quite similar to the one used by Franks in Lemma 5.1 of [12] to move $\psi\left(D^{2}\right)$ into general position under $\mathcal{L}(\Omega)$. Starting with $U_{1}$, we shall alter $\psi\left(D^{2}\right)$ consecutively, leaving a general position of previous steps. As a result, we obtain $\psi\left(D^{2}\right)$ in general position under $\mathcal{L}(\Omega)$, i.e., $\psi\left(D^{2}\right)$ is transverse to the leaves of $\mathcal{L}(\Omega)$ except for isolated generic tangencies of saddle type or center type. Hence, one get the lamination $\mathcal{L}_{D^{2}}=\psi^{-1}\left(\mathcal{L}(\Omega) \cap \psi\left(D^{2}\right)\right)$. Moving $\psi\left(D^{2}\right)$ slightly, one can assume that there are no two singularities which are joined by a leaf of $\mathcal{L}_{D^{2}}$.

We now apply Poincaré-Bendixon theory to this situation [6]. By condition, $\mathcal{L}(\Omega) \cap T \neq \emptyset$. Take a point $x_{0} \in \partial D^{2}$ such that $\psi\left(x_{0}\right) \in \mathcal{L}(\Omega) \cap T$. Let $l$ be the leaf crossing $\partial D^{2}$ at $x_{0}$, and $[0, \infty) \rightarrow l$ a parametrization of $l$ such that 0 corresponds to $x_{0}$. Since $T$ intersects oriently all leaves of $\mathcal{L}(\Omega)$, we see that $l$ never intersects $\partial D^{2}$ after $x_{0}$. Hence, the $\omega$-limit set $\omega(l)$ of $l$ is non-empty and belongs to $D^{2}$. Without loss of generality we can assume that $\omega(l)$ is not a singularity, because $\mathcal{L}_{D^{2}}$ has a finite number of singularities and the intersection $\mathcal{L}(\Omega) \cap T$ is homeomorphic to a Cantor set (see Lemma 1.10).

Since the support of a lamination is a closed set, $\omega(l)$ is closed and belongs to the lamination $\mathcal{L}_{D^{2}}$. Moreover, $\omega(l)$ consists of one-dimensional leaves and singularities of $\mathcal{L}_{D^{2}}$ [6]. Let $l_{0} \subset \omega(l)$ be a one-dimensional leaf. Such a leaf exists, because $\omega(l)$ is not a unique singularity. First, suppose that the limit set $\omega\left(l_{0}\right)$ of $l_{0}$ has no singularities. According to [6], $\omega\left(l_{0}\right)$ does not contain one-dimensional leaves except $l_{0}$. Hence, $l_{0}$ is a closed leaf, and thus $l$ tends spirally to $l_{0}$. Let $L_{0}$ be the leaf of $\mathcal{L}(\Omega)$ with $\psi\left(l_{0}\right) \subset L_{0}$. Since $l_{0} \subset \omega(l), \psi\left(l_{0}\right)$ represents a nontrivial holonomy element of $L_{0}$. But this contradicts Lemma 4 .4 because each leaf of $\mathcal{L}(\Omega)$ is simply connected.

Suppose $\omega\left(l_{0}\right)$ contains singularities. Then $\omega\left(l_{0}\right)$ contains only one singularity, say $s_{0}$. Hence, $\omega\left(l_{0}\right)$ is either a separatrix loop of $s_{0}$ or is a figure eight with center at $s_{0}$. In either case, $\psi\left(\omega\left(l_{0}\right)\right)$ represents a nontrivial holonomy element of the leaf that $\psi\left(\omega\left(l_{0}\right)\right)$ belongs to. Again, this contradicts Lemma 4.4

Corollary 4.3. Let $C$ be a simple closed curve which is a union of some arc $a \subset W^{u}(x), x \in \Omega$, and a segment $s \subset W^{s}(x)$ belonging to some neighborhood of $x$ with a local product structure. Then $C$ is not homotopic to zero in $M$. 
Proof. By the orientability of $\Omega, s$ intersects oriently the leaves of the lamination $\mathcal{L}(\Omega)$. Therefore one can deform $C$ to obtain a simple closed curve $T$ that is homotopic to $C$ and intersects oriently the leaves of the lamination $\mathcal{L}(\Omega)$ [3]. It follows from Lemma 4.5 that $T$, and as a consequence $C$, is not homotopic to zero in $M$.

Corollary 4.4. Suppose $\Omega^{\prime}$ is an orientable codimension one expanding attractor of an A-diffeomorphism $f: M \rightarrow M$. Then there are no closed balls $E \subset M$ such that $\Omega^{\prime} \subset E$.

Corollary 4.5. Suppose $\Omega$ is a codimension one orientable expanding attractor of an A-diffeomorphism $f: M \rightarrow M$ and $\left\{p_{i}, q_{i}\right\}_{i=1}^{k}$ are pairs of associated boundary periodic points of $\Omega$. If each characteristic sphere $S_{p_{i} q_{i}}$ of $\Omega$ bounds a ball in $M$, then $\Omega$ is the only orientable codimension one pseudotame basic set of $f$.

\section{TOPOLOGICAL STRUCTURE OF UNIVERSAL COVERING}

In this section we keep the notation of sections 2 and 4 . In particular, $\Omega$ is an expanding codimension one orientable attractor of an $A$-diffeomorphism $f: M \rightarrow$ $M$, and $\bar{f}: \bar{M} \rightarrow \bar{M}$ is a lift of $f$ under the universal covering map $\pi: \bar{M} \rightarrow M$.

Lemma 5.1. Suppose $\Omega$ is a codimension one orientable expanding attractor and $\bar{\Omega}=\pi^{-1}(\Omega)$. Then the following properties hold:

1) For any $\bar{x}, \bar{y} \in \bar{\Omega}$, there is at most one point in $w^{u}(\bar{x}) \cap w^{s}(\bar{y})$.

2) For any point $\bar{x} \in \bar{\Omega}$, the inclusion $w^{u}(\bar{x}) \subset \bar{M}$ is an embedding.

3) For any point $\bar{x} \in \bar{\Omega}$, the inclusion $w^{u}(\bar{x}) \subset \bar{M}$ is a proper map, i.e., given any compact set $K \subset \bar{M}$, the intersection $K \cap w^{u}(\bar{x})$ is a compact subset in the topology of the unstable manifold $w^{u}(\bar{x}) \cdot 1$

4) For any point $\bar{x} \in \bar{\Omega}$, the set $\bar{M}-w^{u}(\bar{x})$ has two connected component (in other words, $w^{u}(\bar{x})$ divides $\left.\bar{M}\right)$.

Proof. Our proof of the first statement follows the proof of Lemma 5.1 of 12 . Assume the contrary. Then there exist $\bar{x}, \bar{y} \in \bar{\Omega}$ such that $\bar{x}, \bar{y} \in w^{u}(\bar{x}) \cap w^{s}(\bar{y})$ and $\bar{x} \neq \bar{y}$. Note that $w^{u}(\bar{x})=w^{u}(\bar{y})$ and $w^{s}(\bar{x})=w^{s}(\bar{y})$ in this case. Let $[\bar{x}, \bar{y}]^{u} \subset w^{u}(\bar{x})$ be an arc that joins $\bar{x}$ and $\bar{y}$ in $w^{u}(\bar{x})$ such that $(\bar{x}, \bar{y})^{u} \cap(\bar{x}, \bar{y})^{s}=\emptyset$. Such an arc exists because the one-dimensional stable manifold $w^{s}(\bar{x})$ intersects the $(n-1)$-unstable manifold $w^{u}(\bar{x})$ transversally. Hence, $\bar{A}=[\bar{x}, \bar{y}]^{u} \cup[\bar{x}, \bar{y}]^{s}$ is a simple closed curve.

Since $\bar{M}$ is simply connected, $\bar{A}$ is contractible. This implies that $\pi(\bar{A})=A$ is a simple closed curve, which is homotopic to zero in $M$ because every invariant manifold of $\bar{\Omega}$ has no congruent points. Since the points $x=\pi(\bar{x}), y=\pi(\bar{y})$ belong to the same stable manifold, it follows that there is a natural number $j$ such that the arc $\left[f^{j}(x), f^{j}(y)\right]^{s} \subset f^{j}(A)$ belongs to a neighborhood with a local product structure. Obviously, $f^{j}(A)$ is homotopic to zero. We get a contradiction with Corollary 4.3. This concludes the proof of the first statement.

In fact, the second statement follows from the first one, because any point of $w^{u}(\bar{x})$ has a neighborhood with a local product structure.

Let us prove item (3). Assume the contrary. Then there is a sequence of points $\bar{z}_{k} \in w^{u}(\bar{x})$ such that $\bar{z}_{k} \rightarrow z \in K$ as $k \rightarrow \infty$, and this sequence is unbounded in

\footnotetext{
${ }^{1}$ Note that item 2) does not imply item [3), because $w^{u}(\bar{x})$ is not a compact set in $\bar{M}$.
} 
the topology of $w^{u}(\bar{x})$. According to [27], $\mathcal{L}(\bar{\Omega})$ is a codimension one lamination consisting of the unstable manifolds of $\bar{\Omega}$. Therefore, $\bar{z} \in \bar{\Omega}$ and there is a neighborhood $U(\bar{z})$ of $\bar{z}$, which is a laminated chart of $\mathcal{L}(\bar{\Omega})$. Without loss of generality one can assume that $U(\bar{z})$ is endowed with a local product structure. Let $\bar{l}_{k}$ be a local leaf containing $\bar{z}_{k}$ in $U(\bar{z})$. Since the sequence $\bar{z}_{k}$ is unbounded in the topology of $w^{u}(\bar{x})$, we see that there are at least two different local leaves $l_{k_{1}} \neq l_{k_{2}}$. As a consequence, there is an arc $\left[\bar{a}_{1}, \bar{a}_{2}\right]^{s} \subset U(\bar{z})$ such that $\bar{a}_{i} \in l_{k_{i}}, i=1,2$. If we join the points $\bar{a}_{1}, \bar{a}_{2}$ with an arc $\bar{l}_{12} \subset w^{u}(\bar{x})$, we get a closed curve $\bar{C}=\bar{l}_{12} \cup\left[\bar{a}_{1}, \bar{a}_{2}\right]^{s}$, which is homotopic to zero because $\bar{M}$ is simply connected. Hence, $\pi(\bar{C})$ is homotopic to zero in $M$. Again this contradicts Corollary 4.3, and so we complete the proof of item 3 .

Finally, we prove item (4). Take an arc $a$ that intersects $w^{u}(\bar{x})$ transversally at a unique point, say $\bar{x}_{a}$, and belongs to some neighborhood with a local product structure. By item (2), such an arc exists. We have to prove that any $\operatorname{arc} b$ connecting the endpoints of $a$ must intersect $w^{u}(\bar{x})$. Assume the contrary. Without loss of generality one can assume that $\bar{C}=a \cup b$ is a simple smooth curve which is homotopic to zero in $\bar{M}$. Then there is a smooth immersion $\psi: D^{2} \rightarrow M$ such that $\psi\left(\partial D^{2}\right)=C$ and $\psi\left(D^{2}\right)$ is transversal to $w^{u}(\bar{x})$, where $D^{2}$ is a closed 2-disk. By item [3), the intersection $w^{u}(\bar{x}) \cap \psi\left(D^{2}\right)$ is a compact one-dimensional submanifold of $w^{u}(\bar{x})$. Hence, $w^{u}(\bar{x}) \cap \psi\left(D^{2}\right)$ consists of finitely many compact curves, each of which is homeomorphic to either a circle or the segment $[0 ; 1]$. By transversality of the intersection $w^{u}(\bar{x}) \cap \psi\left(D^{2}\right)$, every curve which is homeomorphic to [0;1] has endpoints on $\bar{C}$. This contradicts $\bar{x}_{a}$ being the unique point of the intersection $w^{u}(\bar{x}) \cap \bar{C}$.

Remark. Another way to prove item 4 of Lemma 5.1 is an application of corollary 4.2 and Lemma 1 of section 3 of [23], where a similar assertion is proved for closed leaves of codimension one foliation.

Recall that for a given boundary point $\bar{p} \in \bar{\Omega}$, there is a unique boundary point $\bar{q} \in \bar{\Omega}$ such that $\bar{p}$ and $\bar{q}$ are associated (Lemma 4.1). Let $\bar{x} \in \bar{\Omega}$. By definition, put

$$
w^{s *}(\bar{x})=\left\{\begin{aligned}
w^{s}(\bar{x}), & \text { if } w^{s}(\bar{x}) \text { does not contain boundary points of } \bar{\Omega}, \\
w^{s}(\bar{p}) \cup w^{s}(\bar{q}), & \text { if } w^{s}(\bar{x}) \text { contains a boundary point } \bar{p}, \\
& \text { where } \bar{q} \text { is associated with } \bar{p}
\end{aligned}\right.
$$

The following lemma is a generalization of item 1) of Lemma 5.1.

Lemma 5.2. Suppose $\Omega$ is a codimension one orientable expanding attractor and $\bar{\Omega}=\pi^{-1}(\Omega)$. Then for any $\bar{x}, \bar{y} \in \bar{\Omega}$, there is at most one point in $w^{u}(\bar{x}) \cap w^{s *}(\bar{y})$.

Proof. Assume the contrary. Then there exist two points $\bar{x}, \bar{y} \in \bar{\Omega}$ such that the intersection $\left.w^{u}(\bar{x}) \cap w^{s *(} \bar{y}\right)$ contains at least two points, say $\bar{z}_{1}, \bar{z}_{2}$. By Lemma 5.1, $w^{s *}(\bar{y})$ has associated boundary points $\bar{p}, \bar{q}$ so that $w^{s *}(\bar{y})=w^{s}(\bar{p}) \cup w^{s}(\bar{q})$ and $w^{u}(\bar{x}) \cap w^{s}(\bar{p}) \neq \emptyset, w^{u}(\bar{x}) \cap w^{s}(\bar{q}) \neq \emptyset$. Since $\Omega$ has finitely many boundary points, we see that there are points $\bar{a} \in w^{u}(\bar{x})$ arbitrary close to $\bar{z}_{1}$ such that $w^{s}(\bar{a})$ does not contain boundary points of $\bar{\Omega}$. The theorem on the continuous dependence of invariant manifolds on initial conditions implies that $w^{s}(\bar{a})$ intersects $w^{u}(\bar{x})$ near the point $\bar{z}_{2}$. This contradicts Lemma 5.1 
Lemma 5.3. Let $\Omega$ be a $C$-dense codimension one orientable expanding attractor and $\bar{\Omega}=\pi^{-1}(\Omega)$. Suppose there are points $\bar{x}, \bar{y}, \bar{z} \in \bar{\Omega}$ such that the following conditions hold:

- $\bar{y} \in w^{u}(\bar{x}), \bar{z} \in w^{s}(\bar{x})$.

- There is a sequence of points $\bar{x}_{i} \in[\bar{x}, \bar{z})^{s} \cap \bar{\Omega}$ such that $\bar{x}_{i} \rightarrow \bar{z}$ as $i \rightarrow \infty$.

- The intersection $w^{u}\left(\bar{x}_{i}\right) \cap w^{s}(\bar{y})$ consists of a unique point $\bar{y}_{i}$ for every $i \in \mathbb{N}$.

Then the sequence of points $\bar{y}_{i}$ is bounded on $w^{s}(\bar{y})$.

Proof. We follow the proof of Lemma 5.2 in [12. Suppose the contrary. By Lemma 5.1, $\bar{M}-w^{u}(\bar{x})$ has two connected components. Denote by $w^{u+}(\bar{x})$ the component of $\bar{M}-w^{u}(\bar{x})$ which contains $\bar{z}$. According to Lemma [5.1, $[\bar{x}, \bar{z}]^{s} \subset w^{u+}(\bar{x})$. As a consequence, all points $\bar{x}_{i}$ and $\bar{y}_{i}$ belong to $w^{u+}(\bar{x})$. Therefore all $\bar{y}_{i}$ belong to a unique component of $w^{s}(\bar{y})-\bar{y}$, denoted by $w^{s+}(\bar{y})$, because the intersection $w^{u}(\bar{x}) \cap w^{s}(\bar{y})$ consists of the unique point $\bar{y}$. By our assumption, the sequence of points $\bar{y}_{i}$ is unbounded on $w^{s+}(\bar{y})$. Hence, $\pi\left(w^{s+}(\bar{y})\right)$ has no boundary points of $\Omega$. By Lemma 1.8 $\pi\left(w^{s+}(\bar{y})\right) \cap \Omega$ is dense in $\Omega$, because the basic set $\Omega$ is $C$-dense. This shows that $\bigcup_{\eta \in \pi_{1}(M)} \eta\left(w^{s+}(\bar{y})\right)$ is dense in $\bar{\Omega}$. As a consequence, there is $\eta_{0} \in \pi_{1}(M)$ such that $\eta_{0}\left(w^{s+}(\bar{y})\right)$ intersects a local product structure neighborhood of $\bar{x} \in \bar{\Omega}$. Moreover, according to the theorem on the continuous dependence of unstable manifolds on initial conditions, one can choose $\eta_{0} \in \pi_{1}(M)$ so that each unstable manifold $w^{u} \subset \bar{\Omega}$ intersecting $[\bar{x}, \bar{z}]^{s}$ also intersects $\eta_{0}\left(w^{s+}(\bar{y})\right)$.

By definition, put $\alpha=\eta_{0}^{-1} \in \pi_{1}(M)$. It is clear that $\alpha \neq i d$. The argument shows that any unstable manifold intersecting the segment $\alpha\left([\bar{x}, \bar{z}]^{s}\right)$ must intersect $w^{s+}(\bar{y})$.

Let us show that every unstable manifold of $\bar{\Omega}$ intersecting both $\alpha\left([\bar{x}, \bar{z}]^{s}\right)$ and $w^{s+}(\bar{y})$ has to intersect $[\bar{x}, \bar{z}]^{s}$. Take an unstable manifold $w^{u}(\bar{a}), \bar{a} \in \bar{\Omega}$, so that $w^{u}(\bar{a}) \cap \alpha\left([\bar{x}, \bar{z}]^{s}\right) \neq \emptyset, w^{u}(\bar{a}) \cap w^{s+}(\bar{y}) \neq \emptyset$. Since the sequence $\bar{y}_{i}$ is unbounded on $w^{s}(\bar{y})$, it follows that there are points $\bar{y}_{j}, \bar{y}_{j+1}$ such that the point $\bar{a}_{0}=w^{u}(\bar{a}) \cap$ $w^{s+}(\bar{y})$ belongs to the $\operatorname{arc}\left(\bar{y}_{j}, \bar{y}_{j+1}\right)^{s}$ (it is possible that $\bar{y}_{j}=\bar{y}$ with $j=0$ ). By Lemma [5.1, the points $\bar{y}_{j}, \bar{y}_{j+1}$ belong to different components of $\bar{M}-w^{u}(\bar{a})$. Hence the points

$$
\bar{x}_{j}=w^{u}\left(\bar{y}_{j}\right) \cap[\bar{x}, \bar{z}]^{s}, \quad \bar{x}_{j+1}=w^{u}\left(\bar{y}_{j+1}\right) \cap[\bar{x}, \bar{z}]^{s}
$$

belong to different components of $\bar{M}-w^{u}(\bar{a})$ as well (if $j=0$, then $\bar{x}_{j}=\bar{x}$ ). By Lemma $5.1 w^{u}(\bar{a})=w^{u}\left(\bar{a}_{0}\right)$ intersects the segment $[\bar{x}, \bar{z}]^{s}$ between the points $\bar{x}_{j}$ and $\bar{x}_{j+1}$.

This allows us to define a map

$$
\lambda \circ \alpha\left([\bar{x}, \bar{z}]^{s}\right) \cap \bar{\Omega} \rightarrow[\bar{x}, \bar{z}]^{s} \cap \bar{\Omega}
$$

as follows: $\lambda(\bar{a})=[\bar{x}, \bar{z}]^{s} \cap w^{u}(\bar{a})$, where $\bar{a} \in \alpha\left([\bar{x}, \bar{z}]^{s}\right) \cap \bar{\Omega}$. The argument above shows that the map $\lambda$ is well-defined. By Lemma $5.1 \lambda$ is order-preserving and injective. As a consequence, $\lambda$ is continuous. Let us define the map

$$
g: \alpha\left([\bar{x}, \bar{z}]^{s}\right) \cap \bar{\Omega} \rightarrow \alpha\left([\bar{x}, \bar{z}]^{s}\right) \cap \bar{\Omega} \text { by } g(\bar{a})=\alpha^{-1} \circ \lambda(\bar{a}), \quad \bar{a} \in \alpha\left([\bar{x}, \bar{z}]^{s}\right) \cap \bar{\Omega} .
$$

The map $g$ is continuous and order-preserving because $\alpha(\bar{\Omega})=\bar{\Omega}$ and $\lambda$ is continuous and order-preserving. Since $\alpha\left([\bar{x}, \bar{z}]^{s}\right) \cap \bar{\Omega}$ is a compact set, $g$ has a fixed point $\bar{x}_{0} \in \alpha\left([\bar{x}, \bar{z}]^{s}\right) \cap \bar{\Omega}$ which can be gotten as a limit $\lim _{k \rightarrow \infty} g^{k}(\bar{a})$. We have the 
quality $\alpha\left(\bar{x}_{0}\right)=\lambda\left(\bar{x}_{0}\right)$, which contradicts the assertion that the unstable manifold $w^{u}\left(\bar{x}_{0}\right)$ has no congruent points.

Lemma 5.4. Let $\Omega$ be a $C$-dense codimension one orientable expanding attractor and $\bar{\Omega}=\pi^{-1}(\Omega)$. Suppose $\bar{x}, \bar{z} \in \bar{\Omega}$ are points such that $\bar{z} \in w^{s}(\bar{x}) \cap \bar{\Omega}$. Then $w^{s *}(\bar{y}) \cap w^{u}(\bar{z}) \neq \emptyset$ for any point $\bar{y} \in w^{u}(\bar{x})$.

Proof. By definition, put

$$
\mathcal{B} \subset w^{u}(\bar{x})=\left\{\bar{b} \in w^{u}(\bar{x}) \mid w^{s *}(\bar{b}) \cap w^{u}(\bar{z}) \neq \emptyset\right\} .
$$

Clearly, $\mathcal{B} \neq \emptyset$ because $\bar{x} \in \mathcal{B}$. According to the theorem on the continuous dependence of stable and unstable manifolds on initial conditions, $\mathcal{B}$ is open in $w^{u}(\bar{x})$. Let us prove that $\mathcal{B}$ is closed in $w^{u}(\bar{x})$. Take a sequence of points $\bar{a}_{i} \in \mathcal{B}$ such that $\bar{a}_{i} \rightarrow \bar{b}_{0}$ as $i \rightarrow \infty$. By Lemma [5.2, the intersection $w^{s *}\left(\bar{a}_{i}\right) \cap w^{u}(\bar{z})$ is a unique point denoted by $\bar{c}_{i}$. Denote by $w^{u+}(\bar{x})$ the component of $\bar{M}-w^{u}(\bar{x})$ which contains $\bar{z}$. By Lemma $\left[5.1,[\bar{x}, \bar{z}]^{s} \subset w^{u+}(\bar{x})\right.$ and, as a consequence, all points $\bar{c}_{i}$ belong to $w^{u+}(\bar{x})$. Denote $w^{s+}\left(\bar{b}_{0}\right)=w^{s *}\left(\bar{b}_{0}\right) \cap w^{u+}(\bar{x})$.

Suppose that $(\bar{x}, \bar{z})^{s} \cap \bar{\Omega}=\emptyset$; then $(\bar{x}, \bar{z})^{s}=(\bar{x}, \bar{z})_{\emptyset}^{s}$, and $w^{u}(\bar{x}), w^{u}(\bar{z})$ form a 2-bunch of $\bar{\Omega}$. By Lemma 4.1, $\bar{\varphi}_{\overline{p q}}\left(\bar{b}_{0}\right) \in w^{u}(\bar{z})$, where $\bar{p} \in w^{u}(\bar{x}), \bar{q} \in w^{u}(\bar{z})$ are associated points corresponding to the bunch $w^{u}(\bar{x}) \cup w^{u}(\bar{z})$. Hence, $w^{s *}\left(\bar{b}_{0}\right) \cap$ $w^{u}(\bar{z}) \neq \emptyset$ and $\bar{b}_{0} \in \mathcal{B}$.

Suppose now that $(\bar{x}, \bar{z})^{s} \cap \bar{\Omega} \neq \emptyset$. Due to Lemma 4.1 and Lemma 1.10 we can assume that $\bar{z}$ is not an isolated point on $(\bar{x}, \bar{z}]^{s} \cap \bar{\Omega}$. Therefore there exists a monotone sequence of points $\bar{x}_{k} \in(\bar{x}, \bar{z}]^{s} \cap \bar{\Omega}$ such that $\bar{x}_{k} \rightarrow \bar{z}$ as $k \rightarrow \infty$.

Let us show that every unstable manifold $w^{u} \subset \bar{\Omega}$ intersecting $w^{s+}\left(\bar{b}_{0}\right)$ or $\left[\bar{a}_{i}, \bar{c}_{i}\right]^{s}$ intersects $[\bar{x}, \bar{z}]^{s}$. Note that if $w^{u} \subset \bar{\Omega}$ intersects $w^{s+}\left(\bar{b}_{0}\right)$, then $w^{u}$ intersects $\left[\bar{a}_{i}, \bar{c}_{i}\right]^{s}$ for $i$ sufficiently large. By Lemma [5.1, the points $\bar{a}_{i} \in w^{u}(\bar{x})$ and $\bar{c}_{i}=w^{s} *\left(\bar{a}_{i}\right) \cap$ $w^{u}(\bar{z}) \subset w^{u}(\bar{z})$ belong to different components of $\bar{M}-w^{u}$. Hence, $\bar{x}$ and $\bar{z}$ belong to different components of $\bar{M}-w^{u}$ as well. It follows that $w^{u}$ intersects $[\bar{x}, \bar{z}]^{s}$. In particular, every unstable manifold that contains boundary points and intersects $w^{s+}\left(\bar{b}_{0}\right)$ or $\left[\bar{a}_{i}, \bar{c}_{i}\right]^{s}$ has to intersect $[\bar{x}, \bar{z}]^{s}$.

Now let us show that every unstable manifold $w^{u} \subset \bar{\Omega}$ intersecting $[\bar{x}, \bar{z}]^{s}$ intersects both $w^{s+}\left(\bar{b}_{0}\right)$ and $\left[\bar{a}_{i}, \bar{c}_{i}\right]^{s}$. Note that the points $\bar{x}, \bar{z}$ belong to different components of $\bar{M}-w^{u}$ (Lemma 5.1 ). As a consequence, the points $\bar{a}_{i} \in w^{u}(\bar{x})$ and $\bar{c}_{i}=w^{s} *\left(\bar{a}_{i}\right) \cap w^{u}(\bar{z}) \subset w^{u}(\bar{z})$ belong to different components of $\bar{M}-w^{u}$ as well. Assume that $w^{u} \cap\left[\bar{a}_{i}, \bar{c}_{i}\right]^{s}=\emptyset$. Then $\left[\bar{a}_{i}, \bar{c}_{i}\right]^{s}$ has associated points, say $\bar{p}_{i}$ and $\bar{q}_{i}$. The argument above implies that $w^{u}\left(\bar{p}_{i}\right)$ and $w^{u}\left(\bar{q}_{i}\right)$ intersect $[\bar{x}, \bar{z}]^{s}$. The unstable manifold $w^{u}$ cannot intersect $\left[\bar{x}, w^{u}\left(\bar{p}_{i}\right) \cap w^{s}(\bar{x})\right]^{s}$ or $\left[w^{u}\left(\bar{q}_{i}\right) \cap w^{s}(\bar{z}), \bar{z}\right]^{s}$; otherwise $w^{u}$ would intersect $\left[\bar{a}_{i}, \bar{p}_{i}\right]^{s}$ or $\left[\bar{q}_{i}, \bar{c}_{i}\right]^{s}$ respectively. Hence, $w^{u}$ must intersect

$$
\left(w^{u}\left(\bar{p}_{i}\right) \cap w^{s}(\bar{x}), w^{u}\left(\bar{q}_{i}\right) \cap w^{s}(\bar{z})\right)^{s}=\left(w^{u}\left(\bar{p}_{i}\right) \cap w^{s}(\bar{x}), w^{u}\left(\bar{q}_{i}\right) \cap w^{s}(\bar{z})\right)_{\emptyset}^{s},
$$

which is impossible because of Lemma 4.1

Thus, $w^{s+}\left(\bar{b}_{0}\right) \cap w^{u}\left(\bar{x}_{k}\right) \neq \emptyset$. Due to Lemma [5.2, this intersection is a unique point. By definition, put $\bar{y}_{k}=w^{s+}\left(\bar{b}_{0}\right) \cap w^{u}\left(\bar{x}_{k}\right)$. By Lemma [5.3, the sequence $\bar{y}_{k}$ is bounded on $w^{s+}\left(\bar{b}_{0}\right)$. By Lemma 5.1 the sequence $\bar{y}_{k}$ is monotone because the sequence $\bar{x}_{k}$ is monotone. Since $\bar{\Omega}$ is a compact set, we see that $\bar{y}_{k} \rightarrow \bar{y}_{*} \in \bar{\Omega}$.

Let us prove that $w^{u}\left(\bar{y}_{*}\right)=w^{u}(\bar{z})$. Suppose the contrary. Then $w^{u}\left(\bar{y}_{*}\right)$ intersects $(\bar{x}, \bar{z})^{s}$ at some point $\bar{x}_{*}$. Since $\bar{x}_{k} \rightarrow \bar{z}$, there are infinitely many points $\bar{x}_{k} \in$ $\left(\bar{x}_{*}, \bar{z}\right)^{s}$. This contradicts the fact that the $\bar{y}_{k}$ tend monotonically to $\bar{y}_{*}$. Hence, 
$w^{u}\left(\bar{y}_{*}\right)=w^{u}(\bar{z})$. Therefore, $\mathcal{B}$ is closed. Since $w^{u}(\bar{x})$ is a connected set, $w^{u}(\bar{x})=$ $\mathcal{B}$.

Corollary 5.1. Let $\Omega$ be a $C$-dense codimension one orientable expanding attractor and $\bar{\Omega}=\pi^{-1}(\Omega)$. Suppose $w^{s *}(\bar{x})$ and $w^{s *}(\bar{y})$ intersect a leaf of the lamination $\mathcal{L}(\bar{\Omega})$, where $\bar{x}, \bar{y} \in \bar{\Omega}$. Then $w^{s *}(\bar{x})$ and $w^{s *}(\bar{y})$ intersect the same leaves of $\mathcal{L}(\bar{\Omega})$.

Proof. By Lemma 4.1 and the theorem on the continuous dependence of leaves on initial conditions, there is a point $\bar{z} \in w^{u}(\bar{x})$ such that $\pi\left(w^{s}(\bar{z})\right)$ does not contain boundary periodic points of $\Omega$ and $w^{s}(\bar{z}) \cap w^{u}(\bar{y}) \neq \emptyset$. The result now follows from Lemma 5.4

Lemma 5.5. Suppose $\Omega$ is a codimension one orientable expanding attractor such that each characteristic sphere $S_{p_{i} q_{i}}$ of $\Omega$ bounds a closed ball $E_{p_{i} q_{i}} \subset M$. Let $\bar{B}_{\overline{p q}}=w^{u}(\bar{p}) \cup w^{u}(\bar{q})$ be a 2-bunch of $\bar{\Omega}=\pi^{-1}(\Omega)$, where $\bar{p}$ and $\bar{q}$ are associated points of $\pi^{-1}(\Omega)$, and let $\mathcal{J}_{\overline{p q}} \subset \bar{M}$ be the open domain bounded by $w^{u}(\bar{p})$ and $w^{u}(\bar{q})$. Then $\mathcal{J}_{\overline{p q}} \cap \bar{\Omega}=\emptyset$, i.e., the domain $\mathcal{J}_{\overline{p q}}$ has no points of $\bar{\Omega}$.

Proof. By Corollary 4.1, $\pi\left(\bar{B}_{\overline{p q}}\right)=B_{p q}=W^{u}(p) \cup W^{u}(q)$ is a bunch of $\Omega$, where $p=\pi(\bar{p}), q=\pi(\bar{q})$ are periodic associated points. Let $S_{p q}$ be a characteristic sphere corresponding to $B_{p q}$. By Lemma 4.2, there is the characteristic sphere $\bar{S}_{\overline{p q}}$ which corresponds to $\bar{B}_{\overline{p q}}$ and is a lift of $S_{p q}$. Denote by $m$ the common period of $p$, $q$. By condition, $S_{p q}$ bounds the closed ball $E_{p q} \subset M$. The path lifting theorem implies that $\pi^{-1}\left(E_{p q}\right)$ is a union of closed balls. By item 10 of Lemma 5.1, each closed ball cannot contain another one, and thus these balls are pairwise disjoint. The argument shows that $\pi^{-1}\left(f^{m j}\left(E_{p q}\right)\right)$ is also a union of pairwise disjoint closed balls for any $j \geq 0$. Denote by $\bar{E}_{\overline{p q}}^{j} \subset \pi^{-1}\left(f^{m j}\left(E_{p q}\right)\right)$ the closed ball containing the points $\bar{p}, \bar{q}$.

By definition, put

$$
\mathcal{J}_{\overline{p q}}^{\prime}=\bigcup_{j \geq 0} \bar{E}_{\overline{p q}}^{j} .
$$

By Lemma 5.1 $\mathcal{J}_{\overline{p q}}$ is a connected set. Hence, $\mathcal{J}_{\overline{p q}} \cup w^{u}(\bar{p}) \cup w^{u}(\bar{q})$ is a connected set as well. Again by Lemma 5.1 the topological closure of $\mathcal{J}_{\overline{p q}}$ is equal to

$$
\operatorname{clos} \mathcal{J}_{\overline{p q}}=\mathcal{J}_{\overline{p q}} \cup w^{u}(\bar{p}) \cup w^{u}(\bar{q}),
$$

because $w^{u}(\bar{p})$ and $w^{u}(\bar{q})$ are embedded submanifolds of $\bar{M}$.

Evidently, $\mathcal{J}_{\overline{p q}}^{\prime} \subset \operatorname{clos} \mathcal{J}_{\overline{p q}}$. Due to the theorem on the continuous dependence of unstable manifolds on initial conditions, $\mathcal{J}_{\overline{p q}}^{\prime}$ is an open subset of clos $\mathcal{J}_{\overline{p q}}$. Let us show that $\mathcal{J}_{\overline{p q}}^{\prime}$ is a closed subset of $\operatorname{clos} \mathcal{J}_{\overline{p q}}$. Suppose $\bar{a}_{i} \in \operatorname{clos} \mathcal{J}_{\overline{p q}}$ is a sequence of points such that $\bar{a}_{i} \rightarrow \bar{a}_{*}$ as $i \rightarrow \infty$. Then $\bar{a}_{i} \in \bar{E}_{\overline{p q}}^{j(i)}$ for some $j(i) \geq 0$. If the sequence $j(i)$ is bounded, then $\bar{a}_{*} \in \bar{E}_{\overline{p q}}^{j_{*}}$ for some $j_{*}$, because $\bigcup_{j \geq 0} \bar{E}_{\overline{p q}}^{j}$ is a union of increasing balls. If the sequence $j(i)$ is unbounded, then, according to Lemma 3.1, $\bar{a}_{*} \in \bar{\Omega}$. In both cases, $\bar{a}_{*} \in \mathcal{J}_{\overline{p q}}^{\prime}$. Hence, $\mathcal{J}_{\bar{p} \bar{q}}^{\prime}$ is a closed subset of clos $\mathcal{J}_{\overline{p q}}$. Therefore, $\operatorname{clos} \mathcal{J}_{\overline{p q}}=\mathcal{J}_{\overline{p q}}^{\prime}$.

By item (1) of Lemma 5.1 there are no points of $\bar{\Omega}$ inside $\bar{E}_{\overline{p q}}^{j}$ for any $j \geq 0$. The desired result follows.

Lemma 5.6. Suppose $\Omega$ is a codimension one orientable expanding attractor, and every characteristic sphere $S_{p_{i} q_{i}}$ of $\Omega$ bounds a ball in $M$. If the stable manifold 
$W^{s}(x)$ of a point $x \in \Omega$ does not contain boundary periodic points, then any lift $w^{s}(\bar{x})$ of $W^{s}(x)$ intersects all leaves of the lamination $\mathcal{L}(\bar{\Omega})$, where $\bar{x} \in \bar{\Omega}=\pi^{-1}(\Omega)$ is a lift of $x$.

Proof. By Lemma 3.1, $\Omega$ is a $C$-dense basic set. Hence, $W^{s}(x) \cap \Omega$ is dense in $\Omega$. Since $W^{s}(x)$ does not contain boundary periodic points, it follows from Lemma 1.8 that $W^{s+}(x) \cap \Omega$ and $W^{s-}(x) \cap \Omega$ are dense in $\Omega$, where $W^{s \pm}(x)$ are components of $W^{s}(x)-x$. This implies that there exists a sequence of points $\left\{\bar{x}_{i} \in w^{s}(\bar{x}) \cap \bar{\Omega}\right\}_{-\infty}^{+\infty}$ such that

$$
\bigcup_{i=-\infty}^{+\infty}\left[\bar{x}_{-i}, \bar{x}_{i}\right]^{s}=w^{s}(\bar{x}), \quad \ldots \subset\left[\bar{x}_{-i}, \bar{x}_{i}\right]^{s} \subset\left[\bar{x}_{-i-1}, \bar{x}_{i+1}\right]^{s} \subset \ldots
$$

Denote by $\mathcal{J}_{i} \subset \bar{M}$ the closed domain bounded by $w^{u}\left(\bar{x}_{i}\right)$ and $w^{u}\left(\bar{x}_{-i}\right)$. By Lemma [5.1, the domains $\mathcal{J}_{i}$ form a sequence of increasing connected sets $\mathcal{J}_{1} \subset \mathcal{J}_{2} \subset \ldots$, where each inclusion is proper. Therefore, $\bigcup_{i \in \mathbb{Z}} \mathcal{J}_{i}$ is an open set of $\bar{M}$, because every $\mathcal{J}_{i}$ is a topological submanifold. Let us show that $\bigcup_{i \in \mathbb{Z}} \mathcal{J}_{i}$ is a closed set. Suppose $\bar{y}_{i} \rightarrow \bar{z}$ as $i \rightarrow \infty$, where $\bar{y}_{i} \in w^{u}\left(\bar{x}_{i}\right)$. Then $\bar{z} \in \bar{\Omega}$ because $\bar{\Omega}$ is closed. Hence, $w^{s *}(\bar{z})$ intersects $w^{u}\left(\bar{x}_{i}\right)$ for $i$ sufficiently large. By Corollary $5.1 w^{u}(\bar{z})$ intersects $w^{s}(\bar{x})$. Since the sequence $\bar{x}_{i}$ is unbounded on $w^{s}(\bar{x})$, we have $w^{u}(\bar{z}) \subset \mathcal{J}_{j}$ for some $j \in \mathbb{N}$, and so $\bar{z} \in \bigcup_{i \in \mathbb{Z}} \mathcal{J}_{i}$. Thus, $\bigcup_{i \in \mathbb{Z}} \mathcal{J}_{i}$ is a closed set. As a consequence, $\bigcup_{i \in \mathbb{Z}} \mathcal{J}_{i}=\bar{M}$.

It remains to prove that every unstable manifold $w^{u} \subset \bar{\Omega}$ intersects $w^{s}(\bar{x})$ whenever $w^{u} \subset \mathcal{J}_{i}$ for some $i \in \mathbb{N}$. Suppose the contrary. By Lemma $5.1 \mathcal{J}_{i}$ is arcwise connected. Take a path $P \subset \mathcal{J}_{i}$ from a point $\bar{a} \in w^{u}\left(\bar{x}_{i}\right)$ to a point $\bar{b} \in w^{u}$. By definition, put

$$
P_{a}=\left\{\bar{z} \in P \cap \bar{\Omega} \mid w^{u}(\bar{z}) \cap w^{s}(\bar{x}) \neq \emptyset\right\} .
$$

The argument above shows that $P_{a}$ is a closed subset of $P$. Therefore there is a point $\bar{a}_{*} \in P_{a}$ such that $P_{a}$ belongs to the closed segment $\left[\bar{a}, \bar{a}_{*}\right] \subset P$ and $\left(\bar{a}_{*}, \bar{b}\right) \cap P_{a}=\emptyset$. Without loss of generality we can assume that $P$ is transversal to $w^{u}\left(\bar{a}_{*}\right)$ at the point $\bar{a}_{*}$. Then the theorem on the continuous dependence of invariant manifolds on initial conditions implies that $\bar{\Omega}$ does not intersect $\left(\bar{a}_{*}, \bar{b}\right)$ near $\bar{a}_{*}$. Hence, $w^{u}\left(\bar{a}_{*}\right)$ contains a lift of a boundary point, say $\bar{p}$. Let $\bar{q}$ be the point associated with $\bar{p}$. Denote by $\mathcal{J}_{\overline{p q}}$ the closed domain bounded by $w^{u}(\bar{p})$ and $w^{u}(\bar{q})$. By Corollary [5.1, $w^{u}(\bar{q}) \cap w^{s}(\bar{x}) \neq \emptyset$. From our definition of the point $\bar{a}_{*}$, it follows that $w^{u}(\bar{q}) \cap\left(\bar{a}_{*}, \bar{b}\right)=\emptyset$. Since $\bar{\Omega}$ does not intersect $\left(\bar{a}_{*}, \bar{b}\right)$ near $\bar{a}_{*},\left(\bar{a}_{*}, \bar{b}\right) \subset \mathcal{J}_{\overline{p q}}$. Hence, $w^{u} \subset \mathcal{J}_{\overline{p q}}$. On the other hand, according to Lemma 5.5 there are no points of $\bar{\Omega}$ in $\mathcal{J}_{\overline{p q}}$. This contradiction concludes the proof.

Corollary 5.2. Let the conditions of Lemma 5.6 hold. Then $w^{s *}(\bar{z})$ intersects all unstable manifolds of $\bar{\Omega}$ for any $\bar{z} \in \bar{\Omega}$.

Proof. The proof follows from Lemmas 5.5, 5.6 and Corollary5.1 (note that due to Lemma 3.1. $\pi(\bar{\Omega})=\Omega$ is $C$-dense, and so we can apply Lemma [5.5) .

Lemma 5.7. Suppose $\Omega$ is a codimension one orientable expanding attractor and every characteristic sphere $S_{p_{i} q_{i}}$ of $\Omega$ bounds a ball in $M$. Then $\bar{M}$ is homeomorphic to $\mathbb{R}^{n}$.

Proof. This follows from Lemmas 5.1, 5.5. Corollary 5.2, and the fact that every unstable manifold $w^{u}\left(\bar{x}_{0}\right), \bar{x}_{0} \in \Omega$, is homeomorphic to Euclidean space $\mathbb{R}^{n-1}$. 
Theorem 5.1. Let $f$ be an A-diffeomorphism of a closed $n$-manifold $M^{n}(n \geq 3)$, and let $\Omega$ be an orientable expanding attractor of codimension one. Suppose that each characteristic sphere $S_{p_{i} q_{i}}$ of $\Omega$ bounds an $n$-ball $E_{p_{i} q_{i}} \subset M^{n}$. Then:

1) Any basic set different from $\Omega$ belongs to the union $\bigcup_{i=1}^{k} E_{p_{i} q_{i}}$, where $p_{i}$, $q_{i}$ range over all pairs of associated boundary periodic points of $\Omega$.

2) $\Omega$ is the only orientable codimension one pseudotame basic set of $f$.

3) The universal covering $\bar{M}$ of $M^{n}$ is homeomorphic to Euclidean space $\mathbb{R}^{n}$.

4) The fundamental group $\pi_{1}\left(M^{n}\right)$ is a free abelian finitely generated group. In particular, $M^{n}$ is homotopy equivalent to the $n$-torus $T^{n}$.

Proof. Lemma 3.1 implies item 1), because stable manifolds of basic sets are pairwise disjoint. Items 2) and 3) follow from item 21) of Lemma 5.1 and Lemma 5.7 respectively.

By a light modification of the proof of Theorem 5.1 and Corollary 5.2 in [39], one can prove that the fundamental group $\pi_{1}\left(M^{n}\right)$ is isomorphic to $\mathbb{Z}^{n}$. For the reader's convenience we give a sketch of the proof. Take a point $\bar{x} \in \bar{\Omega}$ such that $\pi\left(w^{s}(\bar{x})\right)$ does not contain boundary periodic points of $\Omega$. By Lemmas 5.1 and 5.6 $\bar{w}^{s}(\bar{x})$ intersects every unstable manifold $\bar{w}^{u} \subset \bar{\Omega}$ at a unique point. Then each element $\alpha$ of the fundamental group $\pi_{1}\left(M^{n}\right)$ induces the map $\mathcal{R}(\alpha)$ on $\bar{w}^{s}(\bar{x}) \cap \bar{\Omega}$ as follows:

$$
\bar{z} \mapsto w^{s}(\bar{x}) \cap \alpha\left(w^{u}(\bar{z})\right), \text { where } \bar{z} \in w^{s}(\bar{x}) \cap \bar{\Omega} .
$$

Let $\mathcal{R}\left(\pi_{1}\left(M^{n}\right)\right)$ be the group formed by these maps $\mathcal{R}(\alpha)$, where $\alpha$ ranges over $\pi_{1}\left(M^{n}\right)$. Obviously, the correspondence $\pi_{1}\left(M^{n}\right) \rightarrow \mathcal{R}\left(\pi_{1}\left(M^{n}\right)\right)$ is a homomorphism.

We consider $w^{s}(\bar{x})$ to be a curve with some orientation. Take a point $\bar{z}_{0} \in$ $w^{s}(\bar{x}) \cap \bar{\Omega}$. Clearly there are points $\bar{z}_{1}, \bar{z}_{2} \in w^{s}(\bar{x}) \cap \bar{\Omega}$ such that $\bar{z}_{1}<\bar{z}_{0}<\bar{z}_{2}$. It follows from Lemma 5.1 that $w^{u}\left(\bar{z}_{0}\right)$ belongs to the domain $\mathcal{J}_{12}$ bounded by $w^{u}\left(\bar{z}_{1}\right)$ and $w^{u}\left(\bar{z}_{2}\right)$. By Lemmas [5.1] and [5.6, given any $\mathcal{R}(\alpha)$, the intersection $\mathcal{R}(\alpha)\left(\mathcal{J}_{12}\right) \cap w^{s}(\bar{x})$ is an interval, so that the point $\alpha\left(\bar{z}_{0}\right)$ is between the points $\alpha\left(\bar{z}_{1}\right), \alpha\left(\bar{z}_{2}\right)$. Hence, $\mathcal{R}(\alpha)$ is a continuous map. Again by Lemma 5.1, $\mathcal{R}(\alpha)$ is oneto-one. The argument shows that $\mathcal{R}(\alpha)$ is a homeomorphism $\bar{w}^{s}(\bar{x}) \cap \bar{\Omega} \rightarrow \bar{w}^{s}(\bar{x}) \cap \bar{\Omega}$.

Every $\mathcal{R}(\alpha) \in \mathcal{R}\left(\pi_{1}\left(M^{n}\right)\right), \alpha \neq i d$, is fixed point free, because any $\bar{w}^{u} \in \bar{\Omega}$ has no congruent points. Hence, $\mathcal{R}\left(\pi_{1}\left(M^{n}\right)\right)$ is isomorphic to $\pi_{1}\left(M^{n}\right)$.

Given an $\mathcal{R}(\alpha) \in \mathcal{R}\left(\pi_{1}\left(M^{n}\right)\right)$, let us prove that if $\mathcal{R}(\alpha)\left(t_{0}\right)>t_{0}$ for some point $t_{0} \in w^{s}(\bar{x}) \cap \bar{\Omega}$, then $\mathcal{R}(\alpha)(t)>t$ for every $t \in w^{s}(\bar{x}) \cap \bar{\Omega}$. Assume the contrary. Then there is $t_{0}^{\prime} \in w^{s}(\bar{x}) \cap \bar{\Omega}$ such that $\mathcal{R}(\alpha)\left(t_{0}^{\prime}\right)<t_{0}^{\prime}$. Denote

$$
P_{u}=\left\{t \in w^{s}(\bar{x}) \cap \bar{\Omega} \mid \mathcal{R}(\alpha)(t)>t\right\}, \quad P_{l}=\left\{t \in w^{s}(\bar{x}) \cap \bar{\Omega} \mid \mathcal{R}(\alpha)(t)<t\right\} .
$$

First let us show that both $P_{u}$ and $P_{l}$ are closed. Let $t_{i} \in P_{u}$ be a sequence such that $t_{i} \rightarrow t_{*}$. Then $t_{*} \in w^{s}(\bar{x}) \cap \bar{\Omega}$, because the set $w^{s}(\bar{x}) \cap \bar{\Omega}$ is closed. Since $\mathcal{R}(\alpha)$ is continuous, $\mathcal{R}(\alpha)\left(t_{*}\right) \geq t_{*}$, and hence $\mathcal{R}(\alpha)\left(t_{*}\right)>t_{*}$, because $\mathcal{R}(\alpha)$ is fixed point free. It follows that $P_{u}$ is closed. In the same way one can prove that $P_{l}$ is a closed set as well.

Without loss of generality we can assume that $t_{0}<t_{0}^{\prime}$; otherwise one could take $\mathcal{R}\left(\alpha^{-1}\right)$. Since both $P_{u}$ and $P_{l}$ are closed, it follows that $\sup P_{u} \in P_{u}$ and $\inf P_{l} \in P_{l}$. Moreover, $\left(\sup P_{u}, \inf P_{l}\right)^{s} \cap \bar{\Omega}=\emptyset$. By Lemma $1.7 \sup P_{u}$ belongs to the unstable manifold, say $w^{u}(\bar{p})$, with a boundary point $\bar{p}$ of $\bar{\Omega}$. Similarly, inf $P_{l}$ belongs to $w^{u}(\bar{q})$ with a boundary point $\bar{q}$. By Lemma 4.1, $\bar{p}$ and $\bar{q}$ are associated, since $\left(\sup P_{u}, \inf P_{l}\right)^{s}=\left(\sup P_{u}, \inf P_{l}\right)_{\emptyset}^{s}$. 
Denote by $\mathcal{J}_{\overline{p q}} \subset \bar{M}$ the open domain bounded by $w^{u}(\bar{p})$ and $w^{u}(\bar{q})$. By Lemma 5.5. $\mathcal{J}_{\overline{p q}} \cap \bar{\Omega}=\emptyset$, and thus $\alpha\left(\mathcal{J}_{\overline{p q}}\right) \cap \bar{\Omega}=\emptyset$. As a consequence, $\mathcal{R}(\alpha)\left(\left(\sup P_{u}, \inf P_{l}\right)^{s}\right)$ is an interval with $\mathcal{R}(\alpha)\left(\left(\sup P_{u}, \inf P_{l}\right)^{s}\right) \cap \bar{\Omega}=\emptyset$. It follows that if

$$
\mathcal{R}(\alpha)\left(\sup P_{u}\right)>\inf P_{l},
$$

then

$$
\mathcal{R}(\alpha)\left(\sup P_{l}\right)>\inf P_{l}
$$

Hence,

$$
\mathcal{R}(\alpha)\left(\sup P_{u}\right)=\inf P_{l}, \quad \alpha\left(w^{u}(\bar{p})\right)=w^{u}(\bar{q}) .
$$

Therefore, $\pi\left(w^{u}(\bar{p})\right)=\pi\left(w^{u}(\bar{q})\right)$, and so $\Omega$ has a 1-bunch. This contradicts Corollary 1.3

Thus, $\mathcal{R}(\alpha)(t)>t$ for every $t \in w^{s}(\bar{x}) \cap \bar{\Omega}$ whenever $\mathcal{R}(\alpha)\left(t_{0}\right)>t_{0}$ for some point $t_{0} \in w^{s}(\bar{x}) \cap \bar{\Omega}$. This allows us to introduce an order relation on $\mathcal{R}\left(\pi_{1}\left(M^{n}\right)\right)$ as follows. By definition, put

$$
\mathcal{R}(\alpha)>\mathcal{R}(\beta) \text { if } \mathcal{R}(\alpha)(\bar{z})>\mathcal{R}(\beta)(\bar{z}) \text { for some } \bar{z} \in w^{s}(\bar{x}) \cap \bar{\Omega}, \quad \alpha, \beta \in \pi_{1}(M) .
$$

Note that given any $\mathcal{R}(\alpha)$ and $\mathcal{R}(\beta)$, we have either $\mathcal{R}(\alpha)>\mathcal{R}(\beta)$ or $\mathcal{R}(\alpha)=\mathcal{R}(\beta)$ or $\mathcal{R}(\alpha)<\mathcal{R}(\beta)$. Further, by Lemma 3.1 $\Omega$ is $C$-dense, and so every unstable manifold $W^{u}(x) \subset \Omega$ is dense in $\Omega$. Hence, $\bigcup_{\alpha \in \pi_{1}\left(M^{n}\right)} \alpha\left(w^{u}\right)$ is dense in $\bar{\Omega}$. Therefore, $\mathcal{R}\left(\pi_{1}(M)\right)$ is an Archimedean ordered group, i.e., given any $\mathcal{R}(\alpha) \neq \mathcal{R}(\beta)$, there is an $m$ such that

$$
\mathcal{R}(\alpha)^{m}>\mathcal{R}(\beta) \text { or } \mathcal{R}(\alpha)^{-m}>\mathcal{R}(\beta)
$$

Due to the Hölder theorem on Archimedean ordered groups, $\mathcal{R}\left(\pi_{1}(M)\right)$ is a free abelian group. As $\mathcal{R}\left(\pi_{1}(M)\right) \cong \pi_{1}(M)$ is finitely generated, we see that $\mathcal{R}\left(\pi_{1}(M)\right)$ is isomorphic to an integer lattice $\mathbb{Z}^{k}$ (see, e.g., [34, ch. 2). Item 31) implies that $\pi_{r}\left(M^{n}\right)=0$ for $r \geq 2$, i.e., $M$ is a $K(\pi, 1)$-space. It follows that $k=n$, because $M$ is a compact manifold. Thus,

$$
\pi_{r}\left(M^{n}\right)=0=\pi_{r}\left(T^{n}\right) \text { for } r \geq 2, \text { and } \pi_{1}\left(M^{n}\right)=\mathbb{Z}^{n}=\pi_{1}\left(T^{n}\right) .
$$

Hence, $M^{n}$ is weakly homotopy equivalent to $T^{n}$, and thus $M^{n}$ is homotopy equivalent to $T^{n}$ because $M^{n}$ is a $K(\pi, 1)$-space.

Note that we can get the same assertion using Plykin's theorem on invariant foliations (45], p.93, and Corollary 5.2 in [39]).

\section{ON THE EXISTENCE OF NONTRANSVERSAL INTERSECTIONS}

In this section we keep the notation of section 2, In particular, $f$ is an $A$ diffeomorphism of a closed $n$-manifold $M^{n}(n \geq 3)$ and $\Omega$ is an orientable expanding attractor of codimension one. In the theorem below we consider $S_{p q}$ to be the characteristic sphere corresponding to a 2-bunch $W^{u}(p) \cup W^{u}(q)$ of $\Omega$, where $p$, $q \in \Omega$ are associated boundary periodic points. Recall that $S_{p q}$ is a union of two closed ( $n-1)$-balls $D_{p} \subset W^{u}(p), D_{q} \subset W^{u}(q)$, and the connecting cylinder

$$
C_{p q}=\bigcup_{x \in \partial D_{p}, y \in \partial D_{q}}(x, y)_{\emptyset}^{s} \text {, where } p \in \text { int } D_{p}, q \in \text { int } D_{q} .
$$

Recall also that $D_{p q}=\bigcup_{x \in W^{u}(p)-i n t D_{p}}\left[x, \varphi_{p q}(x)\right]_{\emptyset}^{s}$. 
Theorem 6.1. Suppose $f$ is a structurally stable diffeomorphism of a closed $n$ manifold $M^{n}(n \geq 3)$ and $\Omega$ is a codimension one orientable expanding attractor of $f$. Let $\Omega^{\prime} \neq \Omega$ be a basic set of index $n-1$ such that $W^{u}\left(\Omega^{\prime}\right) \cap D_{p q} \neq \emptyset$, and let $C \subset D_{p q} \cap W^{u}\left(z^{\prime}\right)$ be a component of the intersection $D_{p q} \cap W^{u}\left(z^{\prime}\right)$, where $z^{\prime} \in \Omega^{\prime}$ is a periodic point. Then

$$
W^{u}\left(z^{\prime}\right) \cap C_{p q}=C \cap C_{p q} \neq \emptyset
$$

and this intersection consists of a unique $(n-2)$-sphere, say $S^{n-2}$, that is isotopic to both $S_{p}=\partial D_{p}$ and $S_{q}=\partial D_{q}$ in the cylinder $C_{p q}$. In particular, $S^{n-2}$ divides $C_{p q}$ into two cylinders, each of whose is homeomorphic to $\Sigma^{n-2} \times[0 ; 1]$.

Proof. By Mañé's theorem [35], $f$ satisfies the strong transversality condition. Taking into account Corollary 3.1, we see that $W^{u}\left(\Omega^{\prime}\right) \cap D_{p q} \neq \emptyset$ implies $W^{u}\left(z^{\prime}\right) \cap C_{p q} \neq$ $\emptyset$. For the same reason, $C \cap C_{p q} \neq \emptyset$. By the strong transversality condition, $W^{u}\left(z^{\prime}\right) \cap C_{p q}$ locally is an $(n-2)$-dimensional submanifold which is transversal to every segment $\left(x, \varphi_{p q}(x)\right)_{\emptyset}^{s}, x \in S_{p}=\partial D_{p}$. Let $S$ be a component of the intersection $C \cap C_{p q}$. We have to prove that $S$ is an $(n-2)$-sphere and $W^{u}\left(z^{\prime}\right) \cap C_{p q}=S$.

Recall that according to Lemma 2.7 the triple $\left(D_{p q}, W^{u}(p)-i n t D_{p}, \pi_{p}\right)$ is a trivial fiber bundle with fiber the interval $[0 ; 1]$, where $\pi_{p}: D_{p q} \rightarrow W^{u}(p)-$ int $D_{p}$ is the projection that takes each point $b \in D_{p q}$ to $x \in W^{u}(p)-$ int $D_{p}$ whenever $b \in\left[x, \varphi_{p q}(x)\right]_{\emptyset}^{s} \subset D_{p q}$.

It is convenient to prove the theorem in steps. The end of the proof of a step will be denoted by $\diamond$. First we prove a technical statement.

Step 6.1. Let $x_{0} \in W^{u}(p)-p$. There is a neighborhood $U_{0}$ of $x_{0}$ in $W^{u}(p)$ with the following property: if $\pi_{p}^{-1}\left(x_{0}\right)$ intersects the unstable manifold $W^{u}(\theta)$ of a point $\theta$ that belongs to a basic set $\Theta$ of index one, then any component of $\pi_{p}^{-1}\left(U_{0}\right) \cap W^{u}(\theta)$ is mapped by $\pi_{p}$ homeomorphically onto $U_{0}$.

Proof of Step [6.1] It was proved by Smale [53] that $M^{n}$ is a union of pairwise disjoint unstable manifolds of basic sets. Therefore, by the strong transversality condition, any point $x \in \pi_{p}^{-1}\left(x_{0}\right)$ belongs to either an unstable $n$-manifold or an unstable $(n-1)$-manifold. Denote by $D_{n-1}\left(\right.$ resp. $\left.D_{n}\right)$ the subset of $\pi_{p}^{-1}\left(x_{0}\right)$ which belongs to unstable $(n-1)$-manifolds (resp. $n$-manifolds). Since any unstable $n$ manifold is an open set of $M^{n}, D_{n}$ is an open subset of $\pi_{p}^{-1}\left(x_{0}\right)$. Hence, $D_{n-1}$ is a compact set. It follows that the angles between the arc $\pi_{p}^{-1}\left(x_{0}\right)$ and unstable $(n-1)$-manifolds through $D_{n-1}$ are separated from zero by a positive constant. This proves the step, because these unstable manifolds through $D_{n-1}$ are locally pairwise disjoint and $D_{n-1}$ is a compact set.

Below, when we say that a set $A \subset W^{u}(p)-i n t D_{p}$ is open in $W^{u}(p)-i n t D_{p}$ we mean the topology induced by the intrinsic topology of $W^{u}(p)$ on $W^{u}(p)-$ int $D_{p}$ as a subset. Denote by $\left.\pi_{p}\right|_{C}: C \rightarrow W^{u}(p)-$ int $D_{p}$ the restriction of $\pi_{p}$ on $C$.

Step 6.2. The set $\pi_{p}(C)$ is open in $W^{u}(p)-$ int $D_{p}$, and $\left.\pi_{p}\right|_{C}$ is a covering map on its image $\pi_{p}(C)$.

Proof of Step 6.2 Take a point $x \in \pi_{p}(C)$. By the strong transversality condition, the intersection $\pi_{p}^{-1}(x) \cap C$ consists of at most a countable set of points $x_{i} \in D_{p q}$. Obviously, each $x_{i}$ is an interior point of $W^{u}\left(z^{\prime}\right)$, and so is an interior point of $C$ in the topology induced by $D_{p q}$ in $C \subset W^{u}\left(z^{\prime}\right) \cap D_{p q}$ as a subset. Hence, $\left.\pi_{p}\right|_{C}\left(x_{i}\right)$ is 
an interior point of $\pi_{p}(C)$, because $\left.\pi_{p}\right|_{C}$ is a local homeomorphism at each of these points $x_{i}$. As a consequence, $\pi_{p}(C)$ is open in $W^{u}(p)-$ int $D_{p}$.

Let $U(x) \subset W^{u}(p)-$ int $D_{p}$ be a neighborhood of $x$ in $W^{u}(p)-$ int $D_{p}$ which satisfies step 6.1, and $U\left(x_{i}\right)$ the component of $C \cap \pi_{p}^{-1}(U(x))$ that contains the point $x_{i}$. Each $U\left(x_{i}\right)$ is a neighborhood of $x_{i}$, because $\left.\pi_{p}\right|_{C}$ is a continuous map. It follows from step 6.1 that the neighborhoods $U\left(x_{i}\right)$ are pairwise disjoint and the restriction $\left.\pi_{p}\right|_{C \cap U\left(x_{i}\right)}$ is a homeomorphism onto $U(x)$. This completes the proof. $\diamond$

Obviously, given any set $N \subset W^{u}(p)-i n t D_{p}$ the restriction $\left.\pi_{p}\right|_{\pi_{p}^{-1}(N)}$ is a trivial fiber bundle with fiber the interval $[0 ; 1]$. Hence the restriction

$$
\left.P \stackrel{\text { def }}{=} \pi_{p}\right|_{\operatorname{clos} C_{p q}}: \operatorname{clos} C_{p q} \rightarrow S_{p}^{n-2}, \quad \operatorname{clos} C_{p q}=C_{p q} \cup S_{p}^{n-2} \cup S_{q}^{n-2},
$$

is a trivial fiber bundle with fiber the interval $[0 ; 1]$ as well, because $\operatorname{clos} C_{p q}=$ $\pi_{p}^{-1}\left(S_{p}^{n-2}\right)$.

Step 6.3. $\left.\pi_{p}\right|_{C}: C \rightarrow W^{u}(p)-$ int $D_{p}$ is a surjection i.e., $\pi_{p}(C)=W^{u}(p)-i n t D_{p}$. In particular, $\left.P\right|_{S}: S \rightarrow S_{p}^{n-2}$ is a surjection as well, $P(S)=S_{p}^{n-2}$.

Proof of Step 6.3 Assume the contrary. Then by step $\left.6.2 \partial \pi_{p}\right|_{C}(C) \neq \emptyset$, and so there is a point $\left.x_{0} \in \partial \pi_{p}\right|_{C}(C)-\left.\pi_{p}\right|_{C}(C)$. It follows we shall obtain that there is a sequence of points $a_{i} \in D_{p q} \cap C$ such that $a_{i} \rightarrow a_{*} \in D_{p q}-C$ and $\left.\pi_{p}\right|_{C}\left(a_{i}\right) \rightarrow x_{0}$ as $i \rightarrow \infty$.

Let $U\left(x_{0}\right) \subset W^{u}(p)-$ int $D_{p}$ be a neighborhood of $x_{0}$ in $W^{u}(p)-$ int $D_{p}$ which satisfies step 6.1. Then the neighborhood $U=\pi_{p}^{-1}\left(U\left(x_{0}\right)\right)$ is filled by unstable $n$-manifolds and unstable $(n-1)$-manifolds necessarily intersecting $\pi_{p}^{-1}\left(x_{0}\right)$. Since $a_{i} \rightarrow a_{*}$, we see that $a_{i} \in U$ beginning with some index $i_{0}$. Hence, $C$ has to intersect $\pi_{p}^{-1}\left(x_{0}\right)$. Thus, $\left.x_{0} \notin \partial \pi_{p}\right|_{C}(C)$, because $\left.\pi_{p}\right|_{C}$ is a local homeomorphism. This contradiction concludes the proof.

Step 6.4. If $n \geq 4$, then $\left.\pi_{p}\right|_{C}: C \rightarrow W^{u}(p)-$ int $D_{p}$ is a homeomorphism, and $\pi_{p}^{-1}(x) \cap C$ consists of a unique point for every $x \in W^{u}(p)-i n t D_{p}$. In particular, $\left.P\right|_{S}: S \rightarrow S_{p}^{n-2}$ is a homeomorphism and $S$ is an $(n-2)$-sphere (we recall that $S$ is a component of the intersection $\left.C \cap C_{p q}\right)$.

Proof of Step 6.4 It follows from steps 6.2 and 6.3 that $\left.\pi_{p}\right|_{C}: C \rightarrow W^{u}(p)-$ int $D_{p}$ is a cover. Since $n \geq 4, \pi_{1}\left(S_{p}^{n-2}\right)=\pi_{1}\left(W^{u}(p)-i n t D_{p}\right)=0$. Hence, $\left.\pi_{p}\right|_{C}$ : $C \rightarrow W^{u}(p)-$ int $D_{p}$ is a trivial cover (see, e.g., [54], section 13.9), i.e., $C$ is homeomorphic to the product of $W^{u}(p)-$ int $D_{p}$ and an at most countable set of points. Taking into account that $C$ is connected, it follows that the fiber of $\left.\pi_{p}\right|_{C}$ is trivial as well, i.e., consists of a unique point. Therefore $\left.\pi_{p}\right|_{C}$ is a homeomorphism $C \rightarrow W^{u}(p)-\operatorname{int} D_{p}$. By construction of $\left.\pi_{p}\right|_{C},\left.S \cap \pi_{p}\right|_{C} ^{-1}(x)$ is a unique point for any $x \in W^{u}(p)-$ int $D_{p}$.

Before we prove the similar statement for $n=3$ we have to prove that some special family of curves on the cylinder $C_{p q}$ satisfies the theorem on continuous dependence on initial conditions. Recall that, due to the strong transversality condition, $C_{p q}$ intersects 2-dimensional and 3-dimensional unstable invariant manifolds only when $n=3$. Denote by $D_{2} \subset C_{p q}$ the set of the intersections $C_{p q}$ with 2dimensional unstable invariant manifolds. Again, due to the strong transversality condition, any 2-dimensional unstable invariant manifold intersects $C_{p q}$ in a curve. Such curves form a family, denoted by $\mathcal{F}$. 


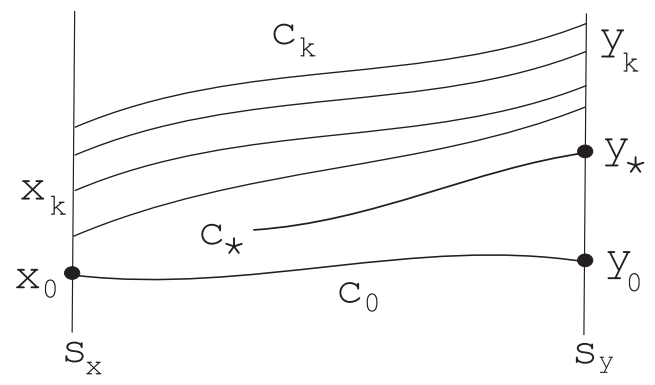

FiguRE 1 . The family $\mathcal{F}$.

Step 6.5. The family $\mathcal{F}$ satisfies the theorem on continuous dependence on initial conditions.

Proof of Step 6.5 Take a curve $c_{0} \in \mathcal{F}$ and any points $x_{0}, y_{0} \in c_{0}$. Denote by $s_{x}$ (resp. $s_{y}$ ) the fiber of the fiber bundle $\pi_{p}$ through $x_{0}$ (resp. $y_{0}$ ), shown in Figure 1

Let $x_{k} \in D_{2}$ be any sequence of points converging to $x_{0}$ as $k \rightarrow \infty$, and denote by $c_{k} \in \mathcal{F}$ the curve through $x_{k}$. By step 6.3 every curve $c_{k}$ intersects both $s_{x}$ and $s_{y}$. Therefore without loss of generality we can assume that $x_{k} \in s_{x}$ and the points $x_{k}$ converge to $x_{0}$ monotonically on $s_{x}$. Let $\left[x_{k}, y_{k}\right] \subset c_{k}$ be the segment with endpoints $x_{k}$ and $y_{k} \in s_{y}$ such that $\pi_{p}\left(\left[x_{k}, y_{k}\right]\right)=\pi_{p}\left(\left[x_{0}, y_{0}\right]\right)$ for any $k$; see Figure 1, where the segment $\left[x_{0}, y_{0}\right] \subset c_{0}$ is mapped by $\pi_{p}$ onto $\pi_{p}\left(\left[x_{0}, y_{0}\right]\right)$ homeomorphically. Then the sequence $y_{k}$ is monotone on $s_{y}$. We have to prove that $y_{k} \rightarrow y_{0}$ as $k \rightarrow \infty$.

Suppose the contrary. Then, passing to a subsequence if necessary, one can assume that $y_{k} \rightarrow y_{*}$ as $k \rightarrow \infty$ for some point $y_{*} \in s_{y}$ that is between $y_{1}$ and $y_{0}, y_{*} \neq y_{0}$. Since $D_{2}$ is a compact set, $y_{*} \in D_{2}$, and thus there is a curve, say $c_{*} \in \mathcal{F}$, through $y_{*}$. By step $6.3 c_{*}$ intersects $s_{x}$. Take the segment $\left[x_{*}, y_{*}\right] \subset c_{*}$ such that $\pi_{p}\left(\left[x_{*}, y_{*}\right]\right)=\pi_{p}\left(\left[x_{0}, y_{0}\right]\right)$, where $x_{*} \in s_{x}$. Then $x_{*} \neq x_{0}$, since $y_{*} \neq y_{0}$. This is impossible because the sequence $y_{k}$ is monotone on $s_{y}$, and thus there are no points $y_{k}$ on the segment $\left[y_{*}, y_{0}\right] \subset s_{y}$ between $y_{*}, y_{0}$.

Step 6.6. Every component $S$ of $C \cap C_{p q}$ is an (n-2)-sphere. Moreover, $S \cap P^{-1}(x)$ is a unique point for any $x \in S_{p}^{n-2}$.

Proof of Step 6.6 By step 6.4, it remains to consider the case $n=3$. Rename $S=c$ and assume that the curve $c \subset W^{u}\left(z^{\prime}\right) \cap C_{p q}$ is not homeomorphic to a circle. Hence, $c$ has a point of accumulation, say $m_{0} \in C_{p q}$, i.e., there is a sequence of points $m_{i} \in c$ such that $m_{i} \rightarrow m_{0}$ as $i \rightarrow \infty$. Take a neighborhood $U\left(m_{0}\right) \subset C_{p q}$ of $m_{0}$ that is a trivial bundle over the open segment $P\left(U\left(m_{0}\right)\right), P\left(m_{0}\right) \in P\left(U\left(m_{0}\right)\right)$, with fibers the arcs of stable manifolds of points $x \in P\left(U\left(m_{0}\right)\right)$. Let $W_{0}^{s} \subset U\left(m_{0}\right)$ be the component of $W^{s}\left(P\left(m_{0}\right)\right) \cap U\left(m_{0}\right)$ through $m_{0}$. Denote by $K\left(m_{i}\right)$ the component of $c \cap U\left(m_{0}\right)$ containing the point $m_{i}$. By step 6.3, we can assume that $K\left(m_{i}\right)$ intersects $W_{0}^{s}$. Since $K\left(m_{i}\right)$ is transversal to the fibers of the trivial fiber bundle $U\left(m_{0}\right)$, $K\left(m_{i}\right) \cap W_{0}^{s}$ is a unique point. Then without loss of generality one can assume that $K\left(m_{i}\right) \cap W_{0}^{s}=m_{i}$. Moreover, we can assume that the components $K\left(m_{i}\right)$ are pairwise disjoint, because $m_{i} \rightarrow m_{0}$ as $i \rightarrow \infty$. Denote by $C\left(m_{i}, m_{i+1}\right)$ the arc of $c$ with endpoints $m_{i}, m_{i+1}$ and by $C_{i, i+1}$ the closed curve which is the union of $C\left(m_{i}, m_{i+1}\right)$ and the segment $\left(m_{i}, m_{i+1}\right) \subset W_{0}^{s}$. Since $C\left(m_{i}, m_{i+1}\right)$ is transversal to 
the fibers of the fiber bundle $P: \operatorname{clos} C_{p q} \rightarrow S_{p}^{n-2}$ and $P\left(m_{i}\right)=P\left(m_{i+1}\right)$, it follows that $C_{i, i+1}$ is not homotopic to zero on the cylinder $\operatorname{clos} C_{p q}$ and $P\left(C_{i, i+1}\right)=S_{p}^{n-2}$. By the strong transversality condition, $C\left(m_{i}, m_{i+1}\right)$ does not intersect $P^{-1}\left(P\left(m_{0}\right)\right)$ except at the points $m_{i}, m_{i+1}$. The argument shows that the sequence $\left\{m_{i}\right\}_{i=1}^{\infty}$ converges monotonically to $m_{0}$, and so $c$ tends spirally to $m_{0}$.

According to [28] and [53], $m_{0} \in W^{u}(\theta)$ for some point $\theta$ of a basic set $\Theta$. By the strong transversality condition, $\operatorname{dim} W^{u}(\theta) \geq 2$. Since $m_{0}$ is a point of accumulation of points $m_{i} \in W^{u}\left(z^{\prime}\right)$ with $\operatorname{dim} W^{u}\left(z^{\prime}\right)=2$, we have $\operatorname{dim} W^{u}(\theta)=2$. Hence the intersection $W^{u}(\theta) \cap \operatorname{clos} C_{p q}$ contains the curve $c_{0}$ through $m_{0}$.

By step 6.5 $c_{0}$ belongs to the limit set of the curve $c$. The argument of PoincaréBendixon theory (see, e.g., 3], 6]) shows that $c_{0}$ is a closed curve, since $c$ and $c_{0}$ are transversal to any arc $P^{-1}(a), a \in S_{p}^{1}$. Therefore, $c_{0}$ bounds a disc on $W^{u}(\theta)$.

Let us show that $\theta$ is a periodic point. If $\Theta=\Omega^{\prime}$, then there is nothing to prove (recall that $z^{\prime}$ is a periodic point and unstable invariant manifolds are pairwise disjoint). So we assume that $\Theta \neq \Omega^{\prime}$. By the theorem on continuous dependence of invariant manifolds on initial conditions, there is $\alpha>0$ such that any unstable manifold $W^{u}(\eta), \eta \in \Theta$, meeting $W_{\alpha}^{s}(\theta)$ must intersect the cylinder $\operatorname{clos} C_{p q}$ through a closed curve near $c_{0}$. Since $c$ tends spirally to $c_{0}$, it follows that at least one component of the set $W_{\alpha}^{s}(\theta)-\theta$ has no intersections with any unstable invariant manifold $W^{u}(\eta), \eta \in \Theta$, because $\Theta \neq \Omega^{\prime}$. Due to Lemma 1.3, $\theta$ is a periodic point.

Using the argument of the proof of Lemma 1.5 in [40], one get that $W^{u}\left(z^{\prime}\right)$ has to meet $W_{\alpha}^{s}(\theta)-\theta$. The $\lambda$-lemma and the theorem on continuous dependence of invariant manifolds on initial conditions implies that $W^{u}\left(z^{\prime}\right)$ has to intersect the cylinder $\operatorname{clos} C_{p q}$ by closed curves near $m_{0}$. This contradiction concludes the proof that $c$ is a closed curve.

It remains to remark that the strong transversality condition implies that $c \cap$ $P^{-1}(x)$ is a unique point for any $x \in S_{p}^{1}$.

Note that all we need in steps 6.2, 6.3, 6.4 and 6.6 was the existence of the set $\pi_{p}^{-1}\left(S_{p}^{n-2}\right) \stackrel{\text { def }}{=} \operatorname{clos} C_{p q}$, i.e., $S_{p}^{n-2}$ is any $(n-2)$-sphere tamely embedded in $W^{u}(p)$ that is not through the point $p$. In the sequel, we specify $S_{p}^{n-2}$ to be a boundary of the $(n-1)$-ball $D_{p}$ belonging to the characteristic sphere $S_{p q}$.

Step 6.7. The $(n-2)$-sphere $S=C \cap C_{p q}$ is isotopic to both $S_{p}^{n-2}$ and $S_{q}^{n-2}$ in the cylinder clos $C_{p q}$. In particular, the inclusion $S \subset D_{p q}$ induces the monomorphism (injective homomorphism) $\pi_{n-2}(S) \rightarrow \pi_{n-2}\left(D_{p q}\right) \cong \mathbb{Z}$. Furthermore, $S$ divides clos $C_{p q}$ into two subcylinders, each of whose is homeomorphic to the cylinder $\Sigma^{n-2} \times[0 ; 1]$.

Proof of Step 6.7 Recall that $P$ is a trivial fiber bundle with fiber the interval $[0 ; 1]$. By step $\left[6.3\right.$ and the strong transversality condition, $\left.P\right|_{S}: S \rightarrow S_{p}^{n-2}$ is a homeomorphism. In particular, every fiber of the fiber bundle $\left(\operatorname{clos} C_{p q}, S_{p}^{n-2}, P\right)$ intersects $S$ at a unique point. Hence there is an isotopy of $S$ into $S_{p}^{n-2}$ along the fibers of the fiber bundle $\left(\operatorname{clos} C_{p q}, S_{p}^{n-2}, P\right)$. Similarly, $S$ is isotopic to $S_{q}^{n-2}$. Since $S$ intersects every fiber of the trivial fiber bundle $\left(\operatorname{clos} C_{p q}, S_{p}^{n-2}, P\right)$ at a unique point and the fiber is the interval [0;1], $S$ divides $\operatorname{clos} C_{p q}$ into two cylinders, each of which is homeomorphic to $\Sigma^{n-2} \times[0 ; 1]$. Combining this with Corollary [2.3, we obtain that $\pi_{n-2}(S) \rightarrow \pi_{n-2}\left(D_{p q}\right)$ is a monomorphism. 
By the theorem on the continuous dependence of invariant manifolds on initial conditions, each $(n-2)$-sphere $S$ of the intersection $W^{u}\left(z^{\prime}\right) \cap C_{p q}$ is tamely embedded in both $W^{u}\left(z^{\prime}\right)$ and $C_{p q}$. Hence, $S$ bounds an open $(n-1)$-ball in $W^{u}\left(z^{\prime}\right)$, denoted by $B_{S}^{n-1}[9]$. An $(n-2)$-sphere $S \subset W^{u}\left(z^{\prime}\right) \cap C_{p q}$ and the corresponding $(n-1)$-ball $B_{S}^{n-1} \subset W^{u}\left(z^{\prime}\right)$ are called minimal, if there are no points of $W^{u}\left(z^{\prime}\right) \cap C_{p q}$ inside $B_{S}^{n-1}$. By the strong transversality condition, every compact subset $K \subset W^{u}\left(z^{\prime}\right)$ intersects finitely many spheres of $W^{u}\left(z^{\prime}\right) \cap C_{p q}$. Hence minimal spheres and corresponding minimal balls exist.

Step 6.8. Let $B_{S}^{n-1} \subset W^{u}\left(z^{\prime}\right)$ be a minimal $(n-1)$-ball bounded by an $(n-2)$ sphere $S \subset W^{u}\left(z^{\prime}\right) \cap C_{p q}$. Then $B_{S}^{n-1} \cap D_{p q}=\emptyset$.

Proof of Step 6.8. Suppose the contrary. Then $B_{S}^{n-1} \subset D_{p q}$, because $B_{S}^{n-1}$ is minimal. Therefore, $S$ defines a trivial element of the group $\pi_{n-2}\left(D_{p q}\right)$. On the other hand, according to step 6.7, $S$ defines a nontrivial element of $\pi_{n-2}\left(D_{p q}\right)$. This contradiction concludes the proof.

There are two possibilities:

1) There exists a minimal $(n-1)$-ball $B^{\prime} \subset W^{u}\left(z^{\prime}\right)$ containing the point $z^{\prime}$.

2) No minimal ball contains $z^{\prime}$.

Consider case 1). Without loss of generality one can assume that $f\left(z^{\prime}\right)=z^{\prime}$, $f(p)=p$, and $f(q)=q$; otherwise we take a some iteration $f^{k}$. Moreover, we shall suppose that the restriction $\left.f\right|_{W^{u}\left(z^{\prime}\right)}$ is an orientation-preserving diffeomorphism.

Recall that a homeomorphism $\psi: M \rightarrow M$ is stable in the Bing sense, if $\psi$ can be represented as a superposition $\psi=\psi_{1} \circ \cdots \circ \psi_{k}$, where every $\psi_{i}, 1 \leq i \leq k$, is fixed inside of some open domain $D_{i} \subset M$. It is well known that an orientationpreserving diffeomorphism is stable in the Bing sense.

Since $f\left(C_{p q}\right) \subset D_{p q}$, we have $f(S) \cap S=\emptyset$, where the $(n-2)$-sphere $S$ bounds $B^{\prime}$ on $W^{u}\left(z^{\prime}\right)$. Hence, $S$ is inside of $f(S)$, because $z^{\prime} \in B^{\prime}$ is the hyperbolic source of the restriction $\left.f\right|_{W^{u}\left(z^{\prime}\right)}$. Taking 10 into account, we get that the $(n-2)$-spheres $S, f(S)$ bound a closed annulus, say $A \subset W^{u}\left(z^{\prime}\right)$, homeomorphic to $\Sigma^{n-2} \times[0 ; 1]$, because the diffeomorphism $\left.f\right|_{W^{u}\left(z^{\prime}\right)}$ is stable in the Bing sense. Combining this with $f(S) \cap S=\emptyset$, we get that $A$ is a fundamental annulus of $\left.f\right|_{W^{u}\left(z^{\prime}\right)}$, i.e.,

$$
\bigcup_{j \in \mathbb{Z}} f^{j}(A)=W^{u}\left(z^{\prime}\right)-z^{\prime}, \quad \text { int } f^{i}(A) \cap i n t f^{l}(A)=\emptyset, \quad i \neq l .
$$

Moreover, $\bigcup_{j \geq 0} f^{j}(A)=W^{u}\left(z^{\prime}\right)-B^{\prime}$. In particular, $B^{\prime} \cap A=\emptyset$.

Step 6.9. Suppose case 1) holds, i.e., $S$ is a minimal sphere such that $z^{\prime}$ is inside $S$ and $A$ is a fundamental annulus of $\left.f\right|_{W^{u}\left(z^{\prime}\right)}$ bounded by $(n-2)$-spheres $S$ and $f(S)$. Then $A \subset D_{p q}$. To be precise, $A \cap \operatorname{int} D_{p q}=\operatorname{int} A \cup f(S)$.

Proof of Step 6.9 Let $h: \Sigma^{n-2} \times[0 ; 1] \rightarrow A$ be an orientation-preserving homeomorphism such that $h\left(\Sigma^{n-2} \times\{0\}\right)=S$ and $h\left(\Sigma^{n-2} \times\{1\}\right)=f(S)$. By the strong transversality condition, the restriction

$$
\left.P_{A} \stackrel{\text { def }}{=} \pi_{p}\right|_{A \cap D_{p q}}: A \cap D_{p q} \rightarrow W^{u}(p)-\text { int } D_{p}
$$

is a local homeomorphism. Since $B^{\prime} \cap D_{p q}=\emptyset$ (see step 6.8) and $B^{\prime} \cap A=\emptyset$, we see that there is an $\varepsilon>0$ such that $h\left(\Sigma^{n-2} \times[0 ; \varepsilon]\right) \subset D_{p q}$. Therefore,

$$
P_{A}\left(h\left(\Sigma^{n-2} \times[0 ; \varepsilon]\right)\right) \subset W^{u}(p)-i n t D_{p} .
$$


Besides, one can choose $\varepsilon>0$ so that $P_{A}$ takes the annulus $h\left(\Sigma^{n-2} \times[0 ; \varepsilon]\right)$ onto $P_{A}\left(h\left(\Sigma^{n-2} \times[0 ; \varepsilon]\right)\right)$ homeomorphically, because $P_{A}$ is a local homeomorphism and $\left.P_{A}\right|_{S}=\left.P\right|_{S}$ is a homeomorphism $S \rightarrow S_{p}^{n-2}$ (see step 6.6). Hence for any $0 \leq t<\varepsilon$, the $(n-2)$-sphere $P_{A}\left(h\left(\Sigma^{n-2} \times\{t\}\right)\right)$ is inside the $(n-2)$-sphere

$$
P_{A}\left(h\left(\Sigma^{n-2} \times\{\varepsilon\}\right)\right)
$$

on the unstable manifold $W^{u}(p)$.

Let $E \subset[0 ; 1]$ be the set of parameters $t_{0}$ satisfying the following properties:

1) $h\left(\Sigma^{n-2} \times\left[0 ; t_{0}\right]\right) \subset D_{p q}$ and $P_{A}\left(h\left(\Sigma^{n-2} \times\left[0 ; t_{0}\right]\right)\right) \subset W^{u}(p)-$ int $D_{p}$.

2) $P_{A}$ maps the $(n-1)$-annulus $h\left(\Sigma^{n-2} \times\left[0 ; t_{0}\right]\right)$ onto $P_{A}\left(h\left(\Sigma^{n-2} \times\left[0 ; t_{0}\right]\right)\right.$ homeomorphically.

3) Given any $0 \leq t<t_{0}$, the $(n-2)$-sphere $P_{A}\left(h\left(\Sigma^{n-2} \times\{t\}\right)\right)$ is inside the $(n-2)$-sphere $P_{A}\left(h\left(\Sigma^{n-2} \times\left\{t_{0}\right\}\right)\right)$ on $W^{u}(p)$. In particular,

$$
P_{A}\left(h\left(\Sigma^{n-2} \times\left[0 ; t_{0}\right]\right)\right) \subset W^{u}(p)-i n t D_{p} .
$$

The argument above shows that $E$ is a nonempty set of $[0 ; 1]$, because $[0 ; \varepsilon] \subset$ $E$. The strong transversality condition implies that $E$ is an open subset of $[0 ; 1]$. Furthermore, if $t \in E$, then $[0, t] \subset E$. Thus, $E$ is an interval in $[0 ; 1]$ with the left endpoint 0 . Let $t_{*}=\sup E$. Obviously, $t_{*}>0$. By continuity of $h, h\left(\Sigma^{n-2} \times\left\{t_{*}\right\}\right) \subset$ $D_{p q}$ because $D_{p q}$ is closed. Moreover, $h\left(\Sigma^{n-2} \times\left\{t_{*}\right\}\right) \subset \operatorname{int} D_{p q}$ since $[0 ; \varepsilon] \subset E$ for $\varepsilon>0$, and so $t_{*} \geq \varepsilon$.

Let us show that $P_{A}$ maps $h\left(\Sigma^{n-2} \times\left\{t_{*}\right\}\right)$ onto $P_{A}\left(h\left(\Sigma^{n-2} \times\left\{t_{*}\right\}\right)\right)$ as a homeomorphism. For $n \geq 4$, this follows from step 6.4 It remains to prove this fact for $n=3$. Suppose not. Then there are points $a, b \in \Sigma^{1} \times\left\{t_{*}\right\}$ such that

$$
P_{A}(h(a))=P_{A}(h(b)) \stackrel{\text { def }}{=} x_{0}, \quad a \neq b .
$$

It follows that $h\left(\Sigma^{1} \times\left[0 ; t_{*}\right]\right)$ intersects the $\operatorname{arc} \pi_{p}^{-1}\left(x_{0}\right)$ at two points $h(a), h(b)$. By the strong transversality condition, there are 2-balls $D_{a} \subset W^{u}\left(z^{\prime}\right), D_{b} \subset W^{u}\left(z^{\prime}\right)$ containing the points $a, b$ respectively and such that $D_{a} \cap D_{b}=\emptyset$. Moreover, we can assume that $\pi_{p}$ projects $D_{a}$ and $D_{b}$ homeomorphically onto $\pi_{p}\left(D_{a}\right)$ and $\pi_{p}\left(D_{b}\right)$ respectively, and $D_{a} \cup D_{b} \subset$ int $D_{p q}$ because $h(a), h(b) \subset$ int $D_{p q}$.

Take $t, 0<t<t_{*}$, close enough to $t_{*}$ so that the simple closed curve $h\left(\Sigma^{1} \times\{t\}\right)$ intersects both $D_{a}$ and $D_{b}$. Since $P_{A}\left(D_{a}\right)$ and $P_{A}\left(D_{b}\right)$ are 2-balls and $P_{A}\left(h\left(\Sigma^{1} \times\{t\}\right)\right)$ bounds the 2-ball $B_{0}$ in $W^{u}(p)$, it follows that

$$
B_{0} \cup P_{A}\left(\left(D_{a}\right) \cup P_{A}\left(\left(D_{b}\right)\right) \stackrel{\text { def }}{=} Q\right.
$$

contains a simple closed curve, say $S_{0}^{1}$, through $x_{0}$. Furthermore, one can construct $S_{0}^{1}$ so that $S_{0}^{1} \subset Q-D_{p}$. Then $\pi_{p}^{-1}\left(S_{0}^{1}\right)$ is a well defined set homeomorphic to a closed annulus $\Sigma^{1} \times[0 ; 1]$. By construction, the intersection $\pi_{p}^{-1}\left(S_{0}^{1}\right) \cap W^{u}\left(z^{\prime}\right)$ contains the component $S_{0}$ through the points $h(a), h(b)$. This contradicts step 6.6. Thus, $P_{A}$ maps $h\left(\Sigma^{n-2} \times\left\{t_{*}\right\}\right)$ onto $P_{A}\left(h\left(\Sigma^{1} \times\left\{t_{*}\right\}\right)\right)$ homeomorphicaly for any $n \geq 3$.

Taking into account the strong transversality condition, we obtain $t_{*} \in E$. As a consequence, $t_{*}=1$. It follows that $P_{A}\left(h\left(\Sigma^{n-2} \times[0 ; 1]\right)\right) \subset W^{u}(p)-$ int $D_{p}$. Hence, $A \cap \operatorname{int} D_{p q}=\operatorname{int} A \cup f(S)$.

Denote $W^{\prime}=W^{u}\left(z^{\prime}\right)-B^{\prime}$. Step 6.9 implies that $W^{\prime} \subset D_{p q} \subset W^{s}(\Omega)$, because $\bigcup_{j \geq 0} f^{j}(A)=W^{u}\left(z^{\prime}\right)-B^{\prime}$ and $f^{j}\left(D_{p q}\right) \subset D_{p q}$ for any $j \geq 0$. Hence, $C=W^{\prime}$ is a 
unique component of the intersection $D_{p q} \cap W^{u}\left(z^{\prime}\right)$ and $S=C \cap C_{p q}$ is a unique component of $W^{u}\left(z^{\prime}\right) \cap C_{p q}$. This concludes the proof of the theorem in case 1).

Let us consider case 2), i.e., no minimal ball contains $z^{\prime}$. We actually prove that this case does not take place.

Step 6.10. Suppose case 2) holds, i.e., no minimal ball contains $z^{\prime}$. Then any path $\omega \subset W^{u}\left(z^{\prime}\right)$ connecting $z^{\prime}$ and a minimal sphere must intersect spheres of the intersection $W^{u}\left(z^{\prime}\right) \cap C_{p q}$ at interior points of $\omega$,

$$
\operatorname{int} \omega \cap\left(W^{u}\left(z^{\prime}\right) \cap C_{p q}\right) \neq \emptyset \text {. }
$$

Proof of Step 6.10. Let $\omega$ be a path with endpoints $z^{\prime}, z^{\prime \prime} \in S$, where $S \subset W^{u}\left(z^{\prime}\right) \cap$ $C_{p q}$ is a minimal sphere. Assume the contrary. Then $\omega \cap\left(W^{u}\left(z^{\prime}\right) \cap C_{p q}\right)=z^{\prime \prime}$. By step 6.8, $B_{S} \cap D_{p q}=\emptyset$, where $B_{S} \subset W^{u}\left(z^{\prime}\right)$ is the minimal $(n-1)$-ball bounded by $S$. Hence, by the strong transversality condition, some exterior of $S$ near $S$ belongs to $D_{p q}$. Therefore, $\omega \subset D_{p q}$, and as a consequence, $z^{\prime} \subset D_{p q}$. This is impossible. $\diamond$

Step 6.11. Suppose that no minimal ball contains $z^{\prime}$ (i.e., case 2) holds). Then there is a compact domain $Q \subset W^{u}\left(z^{\prime}\right)$ bounded by spheres of $W^{u}\left(z^{\prime}\right) \cap C_{p q}$ (one sphere of $\partial Q$ denoted by $S_{\text {ext }}$, is exterior and the others are inside of $\left.S_{\text {ext }}\right)$ such that $Q \subset D_{p q}$.

Proof of Step 6.11. It is sufficient to prove that there exist a sphere $S^{\prime} \subset W^{u}\left(z^{\prime}\right) \cap$ $C_{p q}$ and a minimal ball $B^{\prime \prime} \subset W^{u}\left(z^{\prime}\right)$ inside $S^{\prime}$ such that there is no sphere of $W^{u}\left(z^{\prime}\right) \cap C_{p q}$ which is inside of $S^{\prime}$ and contains $B^{\prime \prime}$ in its interior. Indeed, if we take the region $R \subset W^{u}\left(z^{\prime}\right)$ bounded by $S^{\prime} \stackrel{\text { def }}{=} S_{\text {ext }}$ and delete from $R$ all balls bounded by spheres of $W^{u}\left(z^{\prime}\right) \cap C_{p q}$ which are inside of $S^{\prime}$, then we obtain the desired domain $Q$, because $Q$ contains some exterior of $B^{\prime \prime}$ that is in $D_{p q}$, due to step 6.8, and $Q$ does not contain spheres of $W^{u}\left(z^{\prime}\right) \cap C_{p q}$ except the boundary $\partial Q$.

Take any minimal ball $B^{\prime} \subset W^{u}\left(z^{\prime}\right)$. By step 6.10, there is a sphere $S \subset$ $W^{u}\left(z^{\prime}\right) \cap C_{p q}$ that separates $z^{\prime}$ and $B^{\prime}$. it follows that either $z^{\prime}$ or $B^{\prime}$ is inside $S$. In the last case, there is nothing to prove, because one could take a smallest sphere that bounds a region with $B^{\prime}$ and is not the boundary of $B^{\prime}$. By steps 6.10, 6.8, and the strong transversality condition, such a sphere exists.

So it remains to consider the case when $z^{\prime}$ is in the ball $B$ bounded by $S$. By the strong transversality condition and compactness of $B \cup S, B$ contains finitely many spheres of the intersection $W^{u}\left(z^{\prime}\right) \cap C_{p q}$. Therefore we can assume that there are no spheres inside $S$ surrounding $z^{\prime}$. Since no minimal ball contains $z^{\prime}, B$ is not a minimal ball. Then $B$ contains a minimal ball, say $B^{\prime \prime}$, bounded by the sphere $S^{\prime \prime}=\partial B^{\prime \prime}$ and such that $B^{\prime \prime}$ is inside $S$. By step 6.8, $B^{\prime \prime} \cap D_{p q}=\emptyset$. Obviously, $z^{\prime} \notin D_{p q}$. By step 6.10, there is a sphere surrounding $B^{\prime \prime}$ but not the point $z^{\prime}$. We get the case considered above. This concludes the proof. Then

Let $Q \subset W^{u}\left(z^{\prime}\right)$ be a compact domain that satisfies the conditions of step 6.11

$$
Q \cap C_{p q}=Q \cap D_{p q}=\partial Q,
$$

because $\partial Q \subset W^{u}\left(z^{\prime}\right) \cap C_{p q}$. By definition, put

$$
\left.P_{Q} \stackrel{\text { def }}{=} \pi_{p}\right|_{Q}: Q \rightarrow W^{u}(p)-i n t D_{p} .
$$

By the strong transversality condition, $\left.P_{Q}\right|_{\text {int } Q}$ is a local homeomorphism. Hence,

$$
\left.P_{Q}\right|_{\text {int } Q}: \operatorname{int} Q \rightarrow P_{Q}(\text { int } Q)
$$




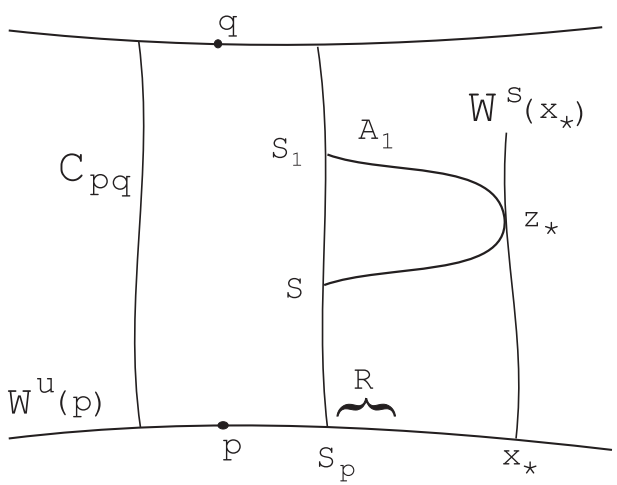

FIGURE $2 . z_{*}$ is the point at which $P_{Q}$ fails to be a local homeomorphism.

is a covering map. The strong transversality condition implies that there is an annulus $A_{1} \subset Q$ with $\partial A_{1} \subset \partial Q$ such that the annulus $P_{Q}\left(A_{1}\right)=R$ has the boundary component $S_{p}$, as shown in Figure 2 It follows that $\partial P_{Q}(Q)-S_{p} \neq \emptyset$, because $Q$ and $P_{Q}(Q)$ are compact sets. Take a point $x_{*} \in \partial P_{Q}(Q)-S_{p}$. There is a point $z_{*} \in Q$ such that $P_{Q}\left(z_{*}\right)=x_{*}$, because $P_{Q}(Q)$ is a compact set, and thus $\partial P_{Q}(Q) \subset P_{Q}(Q)$. Since $x_{*} \in \partial P_{Q}(Q)-S_{p}, z_{*} \in$ int $Q$. It follows that $\left.P_{Q}\right|_{\text {int } Q}$ fails to be a local homeomorphism at the point $z_{*}$. What we have actually proved is that case 2) implies a tangency of invariant manifolds. This is impossible when $f$ is a structurally stable diffeomorphism. This contradiction completes the proof of the theorem.

The main result of this section is the following theorem.

Theorem 6.2. Let $f$ be an A-diffeomorphism of a closed $n$-manifold $M^{n}(n \geq 3)$, and $\Omega$ an orientable expanding attractor of codimension one. Suppose $\Omega^{\prime} \neq \Omega$ is a nontrivial basic set of $f$ such that $W^{u}\left(\Omega^{\prime}\right) \cap W^{s}(\Omega) \neq \emptyset$. Then $f$ is not structurally stable.

Proof. Taking into account the Mañé-Robinson theorem [35, 47, it is sufficient to prove that $f$ does not satisfy the strong transversality condition. Therefore we can assume that Morse's index of every basic set $\Theta$ with $W^{u}(\Theta) \cap W^{s}(\Omega) \neq \emptyset$ is not less than $n-1$. Otherwise there is nothing to prove, since $\operatorname{dim} E_{x}^{s}=1, x \in \Omega$.

Suppose the theorem is not correct; then any stable manifold $W^{s}(x), x \in \Omega$, intersects transversally any unstable manifold $W^{u}(y), y \in N W(f)$. By the condition of the theorem and the paper $\left[28, W^{s}(z) \cap W^{u}\left(z^{\prime}\right) \neq \emptyset\right.$ for some points $z \in \Omega, z^{\prime} \in \Omega^{\prime}$. Hence the unstable manifold $W^{u}\left(z^{\prime}\right)$ is either $(n-1)$-dimensional or $n$-dimensional. If $W^{u}\left(z^{\prime}\right)$ is $n$-dimensional, then $z^{\prime}$ is a periodic point, and so $\Omega^{\prime}$ is trivial. Thus, $W^{u}\left(z^{\prime}\right)$ is $(n-1)$-dimensional. Without loss of generality, we can assume that the points $z$ and $z^{\prime}$ are periodic, because periodic points are dense in basics sets.

Take a point $a \in W^{s}(z) \cap W^{u}\left(z^{\prime}\right)$. Obviously, no point of $W^{s}(z) \cap W^{u}\left(z^{\prime}\right)$ is in $\Omega$, because $\Omega$ is an attractor and consists of unstable manifolds of its points. It follows from Lemma [1.9 that either $a \in\left(x_{a}, y_{a}\right)_{\emptyset}^{s} \subset W^{s}(z)$, where $x_{a} \in W^{u}(p)$, $y_{a} \in W^{u}(q)$ for some associated boundary periodic points $p, q \in \Omega$, or $a \in W_{\emptyset}^{s}(p)$ for some periodic boundary point $p \in \Omega$. Taking into account the theorem on the 
continuous dependence of invariant manifolds on initial conditions, we can assume that $a \in\left(x_{a}, y_{a}\right)_{\emptyset}^{s} \subset W^{s}(z)$.

Let $S_{p q}$ be a characteristic sphere corresponding to the 2-bunch $W^{u}(p) \cup W^{u}(q)$. Recall that $S_{p q}$ is a union of two closed $(n-1)$-balls $D_{p} \subset W^{u}(p), D_{q} \subset W^{u}(q)$ and the connecting cylinder

$$
C_{p q}=\bigcup_{x \in \partial D_{p}, y \in \partial D_{q}}(x, y)_{\emptyset}^{s} \text {, where } p \in \text { int } D_{p}, q \in \text { int } D_{q} .
$$

By construction of a characteristic sphere (see section 2), we can choose $S_{p q}$ so that

$$
\left(x_{a}, y_{a}\right)_{\emptyset}^{s} \in D_{p q}, \text { where } D_{p q}=\bigcup_{x \in W^{u}(p)-\text { int } D_{p}}\left[x, \varphi_{p q}(x)\right]_{\emptyset}^{s} .
$$

Thus, $D_{p q} \cap W^{u}\left(z^{\prime}\right) \neq \emptyset$. By Corollary 3.1] $W^{u}\left(z^{\prime}\right)$ intersects $C_{p q}$.

By Theorem 6.1, the intersection $W^{u}\left(z^{\prime}\right) \cap D_{p q}$ consists of a unique component $C \subset D_{p q} \cap W^{u}\left(z^{\prime}\right)$. Moreover,

$$
W^{u}\left(z^{\prime}\right) \cap C_{p q}=C \cap C_{p q}=S^{n-2}
$$

is an $(n-2)$-sphere that is isotopic to both $S_{p}=\partial D_{p}$ and $S_{q}=\partial D_{q}$ in the cylinder $C_{p q}$. Denote by $B^{\prime} \subset W^{u}\left(z^{\prime}\right)$ the open $(n-1)$-ball bounded by $S^{n-2}$ in $W^{u}\left(z^{\prime}\right)$.

Denote $W^{\prime}=W^{u}\left(z^{\prime}\right)-B^{\prime}$, and let $\Omega_{c}^{\prime}$ be the $C$-dense component of $\Omega^{\prime}$ containing the point $z^{\prime}$. Without loss of generality we can assume that $f\left(\Omega_{c}^{\prime}\right)=\Omega_{c}^{\prime}$ (see [2], [8]). Since $W^{u}\left(z^{\prime}\right) \cap C_{p q}=S^{n-2}, W^{\prime} \subset D_{p q} \subset W^{s}(\Omega)$. Hence, $W^{\prime} \cap \Omega_{c}^{\prime}=\emptyset$. It follows from the nontriviality of $\Omega_{c}^{\prime}$ that the intersection $W^{u}\left(z^{\prime}\right) \cap \Omega_{c}^{\prime}$ must be dense in $\Omega_{c}^{\prime}$. Hence,

$$
W^{u}\left(z^{\prime}\right) \cap \Omega_{c}^{\prime}=\left(B^{\prime} \cup W^{\prime}\right) \cap \Omega_{c}^{\prime}=B^{\prime} \cap \Omega_{c}^{\prime}
$$

is dense in $\Omega_{c}^{\prime}$. On the other hand, $B^{\prime} \cap \Omega_{c}^{\prime}$ is nowhere dense in $\Omega_{c}^{\prime}$, because clos $B^{\prime}$ is a compact set. This contradiction concludes the proof.

Corollary 6.1. Suppose $f$ is a structurally stable A-diffeomorphism of a closed $n$-dimensional manifold $M^{n}(n \geq 3)$, and $\Omega$ is an orientable expanding attractor of codimension one. Let $B$ be a 2-bunch consisting of two unstable manifolds $W^{u}(p)$ and $W^{u}(q)$, where $p, q \in \Omega$ are associated boundary periodic points. Let $S_{p q}$ be the characteristic sphere corresponding to $B$ which is the union of a connecting cylinder $C_{p q}$ and two $(n-1)$-balls $D_{p} \subset W^{u}(p), D_{q} \subset W^{u}(q)$. Suppose $\Theta$ is a basic set of index $n-1$ such that $W^{u}(z) \cap C_{p q}(z \in \Theta)$ is nonempty. Then $\Theta$ is a trivial basic set, $z$ is a periodic point, and $W^{u}(z) \cap C_{p q}$ consists of a unique $(n-2)$ sphere, say $S^{n-2}$, that is isotopic to both $S_{p}=\partial D_{p}$ and $S_{q}=\partial D_{q}$ in the cylinder $C_{p q}$. In particular, $S^{n-2}$ divides $C_{p q}$ into two cylinders that are homeomorphic to $\Sigma^{n-2} \times[0 ; 1]$.

Corollary 6.2. Suppose $f$ is a structurally stable A-diffeomorphism of a closed $n$-dimensional manifold $M^{n}(n \geq 3)$, and $\Omega$ is an orientable expanding attractor of codimension one. Let $B$ be a 2-bunch consisting of two unstable manifolds $W^{u}(p)$ and $W^{u}(q)$, where $p, q \in \Omega$ are associated boundary periodic points. Then both $W_{\emptyset}^{s}(p)$ and $W_{\emptyset}^{s}(q)$ belong to $n$-dimensional unstable manifolds of repelling periodic points.

Proof. Suppose the contrary; then $W^{u}(z) \cap\left(W_{\emptyset}^{s}(p) \cup W_{\emptyset}^{s}(q)\right) \neq \emptyset$ for some point $z \in \Theta$, where the basic set $\Theta$ is of index $n-1$. To be definite, suppose that $W^{u}(z) \cap W_{\emptyset}^{s}(p) \neq \emptyset$. Due to Theorem 6.2, $\Theta$ is trivial, i.e., $z$ is a periodic point. 
As a consequence, $W^{u}(z)$ must intersect $W_{\emptyset}^{s}(p)$ arbitrarily close to $p$. We keep the notation of Corollary 6.1. According to the $\lambda$-lemma, $W^{u}(z)$ must intersect the connecting cylinder $C_{p q}$ in infinitely many components. This contradicts Corollary 6.1 .

\section{ON A TOPOLOGicAl STRUCTURE OF $M^{n}$}

The main result of this section is the following theorem.

Theorem 7.1. Suppose $f$ is a structurally stable diffeomorphism of a closed $n$ manifold $M^{n}(n \geq 3)$, and $\Omega$ is a codimension one orientable expanding attractor of $f$. Then:

1) $M^{n}$ is homotopy equivalent to the $n$-torus $T^{n}$. If $n \neq 4$, then $M^{n}$ is homeomorphic to $T^{n}$.

2) $\Omega$ is the only nontrivial basic set of $f$.

3) $f$ has at least one repelling periodic point.

Proof. Take a bunch $B$ of $\Omega$. Since $\Omega$ is orientable, $B$ is a 2-bunch consisting of unstable manifolds $W^{u}(p)$ and $W^{u}(q)$, where $p$ and $q$ are associated boundary periodic points of $\Omega$; see Corollary 1.3 . Let $S_{p q}$ be the characteristic sphere corresponding to $B$ and consisting of two $(n-1)$-balls $D_{p} \subset W^{u}(p), D_{q} \subset W^{u}(q)$, and connecting $(n-1)$-cylinder $C_{p q} \subset W^{s}(\Omega)$. According to Theorem 5.1, in order to prove the homotopical equivalence of $M^{n}$ and $T^{n}$, we must show that $S_{p q}$ bounds a ball.

Taking some iteration $f^{k}$ if necessary, we can assume without loss of generality that $p$ and $q$ are fixed points. Then Corollary 6.2 implies that $W_{\emptyset}^{s}(p)$ and $W_{\emptyset}^{s}(q)$ belong to unstable $n$-dimensional manifolds of repelling fixed points, say $\alpha_{p}$ and $\alpha_{q}$ respectively. As a consequence, $p$ and $q$ belong to the accessible boundaries $\delta\left(W^{u}\left(\alpha_{p}\right)\right)$ and $\delta\left(W^{u}\left(\alpha_{q}\right)\right)$ respectively. Due to the theorem on the continuous dependence of unstable manifolds on initial conditions, $W^{u}(p)$ and $W^{u}(q)$ belong to $\delta\left(W^{u}\left(\alpha_{p}\right)\right)$ and $\delta\left(W^{u}\left(\alpha_{q}\right)\right)$ respectively as well. Hence, $W^{u}\left(\alpha_{p}\right)$ and $W^{u}\left(\alpha_{q}\right)$ have to intersect the cylinder $C_{p q}$. Obviously, the intersections $W^{u}\left(\alpha_{p}\right) \cap C_{p q}$ and $W^{u}\left(\alpha_{q}\right) \cap C_{p q}$ are open subsets of $C_{p q}$.

Note that $C_{p q}$ can intersect $n$-dimensional or $(n-1)$-dimensional unstable manifolds only, since $f$ is structurally stable and satisfies the strong transversality condition [35. If $C_{p q}$ has no intersections with $(n-1)$-dimensional unstable manifolds, then the connectedness of $C_{p q}$ implies $W^{u}\left(\alpha_{p}\right)=W^{u}\left(\alpha_{q}\right)$ and $\alpha_{p}=\alpha_{q} \stackrel{\text { def }}{=} \alpha$. Hence, $C_{p q} \subset W^{u}(\alpha)$.

For $\varepsilon>0$ sufficiently small, according to the local product structure on $D_{p}$, the set

$$
N\left(D_{p}\right) \stackrel{\text { def }}{=} \bigcup_{x \in D_{p}} W_{\varepsilon}^{s}(x)
$$

is a tubular domain, which we can consider to be a trivial fiber bundle

$$
\left(N\left(D_{p}\right), D_{p}, N\left(D_{p}\right) \rightarrow D_{p}\right) \cong\left(D_{p} \times(-1 ;+1), D_{p}, D_{p} \times(-1 ;+1) \rightarrow D_{p}\right)
$$

with the fiber $W_{\varepsilon}^{s}(x)$ over every point $x \in D_{p}$. Clearly, $D_{p}$ divides $N\left(D_{p}\right)$ into two domains. One of them, say $N^{+}\left(D_{p}\right)$, intersects both $C_{p q}$ and $W_{\emptyset}^{s}(p)$. Combining this with the inclusion $D_{p} \subset \delta\left(W^{u}(\alpha)\right)$, we see that

$$
\operatorname{clos} N^{+}\left(D_{p}\right) \subset D_{p} \cup W^{u}(\alpha) \subset \delta\left(W^{u}(\alpha)\right) \cup W^{u}(\alpha) .
$$


Bearing in mind the natural isomorphism of the fiber bundles $N\left(D_{p}\right)$ and $D_{p} \times$ $(-1 ;+1)$, denote

$$
D_{p, \varepsilon} \cong D_{p} \times\left\{\frac{1}{2}\right\} \subset N^{+}\left(D_{p}\right), \quad C_{p, \varepsilon}=\left(\bigcup_{x \in \partial D_{p}}(x, y)_{\emptyset, \varepsilon}^{s}\right) \cap C_{p q}=N^{+}\left(D_{p}\right) \cap C_{p q} .
$$

It is easy to see that $D_{p, \varepsilon}, D_{p}$, and $C_{p, \varepsilon}$ bound the $n$-ball $B_{p} \subset W^{u}(\alpha)$, and the union $C_{p, \varepsilon} \cup D_{p}$ is an $(n-1)$-ball on the characteristic sphere $S_{p q}$.

Let $D_{q, \varepsilon}, C_{q, \varepsilon}$ be the similar ball and cylinder constructed in some tubular domain $N\left(D_{q}\right)$ near $D_{p}$. Denote

$$
C_{p q, \varepsilon} \stackrel{\text { def }}{=} C_{p q}-\left(C_{p, \varepsilon} \cup C_{q, \varepsilon}\right) .
$$

Then $S_{p q}^{\prime}=C_{p q, \varepsilon} \cup D_{p, \varepsilon} \cup D_{q, \varepsilon}$ is an $(n-1)$-sphere isotopic to $S_{p q}$ in $W^{u}(\alpha)$. Note that $D_{p} \subset \delta\left(W^{u}(\alpha)\right)$ and $D_{q} \subset \delta\left(W^{u}(\alpha)\right)$ are isotopic to $D_{p, \varepsilon}$ and $D_{q, \varepsilon}$ in $B_{p} \subset W^{u}(\alpha)$ and $B_{q} \subset W^{u}(\alpha)$ respectively.

Without loss of generality we can assume that $S_{p q}^{\prime}$ is a tamely embedded sphere. Therefore, $S_{p q}^{\prime}$ bounds a closed $n$-ball, say $B_{p q}^{\prime}$, in $W^{u}(\alpha)$, because $W^{u}(\alpha)$ is homeomorphic to $\mathbb{R}^{n}$. It follows that $S_{p q}$ also bounds the closed $n$-ball $B_{p q}^{\prime} \cup B_{p} \cup B_{q}$ in $W^{u}(\alpha)$. Thus we've proved that if $C_{p q}$ has no intersections with $(n-1)$-dimensional unstable manifolds, then $S_{p q}$ bounds an $n$-ball in $M^{n}$.

Suppose now that $C_{p q}$ intersects $(n-1)$-dimensional unstable manifolds. Due to Theorem 6.2 each of them is an unstable manifold of some saddle periodic point $P_{i}$. Moreover, the orbit of every $P_{i}$ forms a trivial basic set of index $n-1$. There are finitely many of these points, because there are finitely many basic sets. Thus we have finitely many periodic saddle points $P_{1}, \ldots, P_{k}$ such that $W^{u}\left(P_{i}\right) \cap C_{p q} \neq \emptyset$, $1 \leq i \leq k$.

By Corollary 6.1, given a point $P_{i}$, the intersection $W^{u}\left(P_{i}\right) \cap C_{p q}$ consists of a unique $(n-2)$-sphere, say $S_{i}^{n-2}$, that is isotopic to both $S_{p}=\partial D_{p}$ and $S_{q}=\partial D_{q}$ in the cylinder $C_{p q}$. In particular, $S_{i}^{n-2}$ divides $C_{p q}$ into two cylinders that are homeomorphic to $\Sigma^{n-2} \times[0 ; 1]$. Therefore we can rename the points $P_{i}$ if necessary, putting them in order from

$$
S_{0}^{n-2}=W^{u}(p) \cap \partial C_{p q}=\partial D_{p}
$$

to

$$
S_{k+1}^{n-2}=W^{u}(q) \cap \partial C_{p q}=\partial D_{q} .
$$

Rename $\alpha_{p}$ and $\alpha_{q}$ as $\alpha_{0}$ and $\alpha_{k}$ respectively.

Every sphere $S_{i}^{n-2}=W^{u}\left(P_{i}\right) \cap C_{p q}$ is tamely embedded in $W^{u}\left(P_{i}\right)$, and thus it bounds the closed $(n-1)$-ball $D_{i} \subset W^{u}\left(P_{i}\right)$, because $W^{u}\left(P_{i}\right) \cong \mathbb{R}^{n-1}, i=1, \ldots, k$. Since $S_{i}^{n-2}$ and $S_{i+1}^{n-2}$ are isotopic in $C_{p q}$, it follows that they bound the domain $C_{i} \subset C_{p q}$ homeomorphic to the open cylinder $\Sigma^{n-2} \times(0 ; 1), i=0, \ldots, k$. Then the union

$$
S_{i} \stackrel{\text { def }}{=} D_{i} \cup C_{i} \cup D_{i+1}
$$

is an $(n-1)$-sphere tamely embedded in $M^{n}$. By Corollary 6.2 every cylinders $C_{i}$ belongs to an $n$-dimensional unstable manifold of a repelling periodic point, say $\alpha_{i}$. By passing to some iteration of $f$, we can assume that all points $p=P_{0}, P_{1}$, $\ldots, P_{k}, P_{k+1}=q$, and $\alpha_{0}, \ldots, \alpha_{k}$ are fixed. 
Due to the theorem on the continuous dependence of invariant manifolds on initial conditions, the inclusions $C_{i} \subset W^{u}\left(\alpha_{i}\right)$ and $C_{i-1} \subset W^{u}\left(\alpha_{i-1}\right)$ imply

$$
D_{i} \subset \delta\left(W^{u}\left(\alpha_{i}\right)\right) \cap \delta\left(W^{u}\left(\alpha_{i-1}\right)\right) \text { for } i=1, \ldots, k .
$$

Hence, $W^{s}\left(P_{i}\right) \cap W^{u}\left(\alpha_{i}\right) \neq \emptyset$ and $W^{s}\left(P_{i}\right) \cap W^{u}\left(\alpha_{i-1}\right) \neq \emptyset$. Note that $P_{i}$ is a saddle fixed point of index $n-1$. Therefore, $W^{s}\left(P_{i}\right)$ is a one-dimensional curve divided by $P_{i}$ into two curves $W^{s-}\left(P_{i}\right)$ and $W^{s+}\left(P_{i}\right)$. Each of them can't intersect unstable $(n-1)$-manifolds; otherwise $C_{i-1}$ or $C_{i}$ would intersect unstable $(n-1)$-manifolds as well, which is impossible. Thus,

$$
W^{s-}\left(P_{i}\right) \cup W^{s+}\left(P_{i}\right) \subset W^{u}\left(\alpha_{i}\right) \cup W^{u}\left(\alpha_{i-1}\right) .
$$

Using an argument quite similar to the one above for the case $k=0$, one can prove that the $(n-1)$-sphere $S_{0}$ bounds an $n$-ball $B_{0} \subset W^{u}\left(\alpha_{0}\right)$. By the local product structure on $\Omega$, given any point $x \in D_{0}-P_{0}$, the $\operatorname{arc}\left[x, \varphi_{p q}(x)\right]_{\emptyset}^{s}$ enters into $B_{0}$, and has to leave $B_{0}$ because $\varphi_{p q}(x)$ is outside of $B_{0}$ (recall that $D_{0}=D_{p}$ and $\left.P_{0}=p\right)$. Let $\varphi_{01}(x)$ be the first intersection of $\left[x, \varphi_{p q}(x)\right]_{\emptyset}^{s}$ with $S_{0}=\partial B_{0}$ after $x$. By orientability of $\Omega, \varphi_{01}(x) \notin D_{0}$. Hence, $\varphi_{01}(x) \in D_{1}-P_{1}$. Since stable manifolds are pairwise disjoint, we see that

$$
\varphi_{01}: D_{0}-P_{0} \rightarrow D_{1}-P_{1}
$$

is a one-to-one map. It follows that $W^{s-}\left(P_{1}\right)$ and $W^{s+}\left(P_{1}\right)$ do not both intersect $D_{1}-P_{1}$. Therefore there is a unique component of $W^{s}\left(P_{1}\right)-P_{1}$, say $W^{s-}\left(P_{1}\right)$, which belongs to $B_{0} \cap W^{u}\left(\alpha_{0}\right)$. Another component $W^{s+}\left(P_{1}\right)$ belongs to $W^{u}\left(\alpha_{1}\right)$. In particular, $\alpha_{0} \neq \alpha_{1}$.

Continuing this line of reasoning, we see that every $(n-1)$-sphere $S_{i}$ bounds an $n$-ball $B_{i} \subset W^{u}\left(\alpha_{i}\right)$, and the points $\alpha_{0}, \ldots, \alpha_{k}$ are pairwise disjoint. As a consequence, the open balls int $B_{0}, \ldots$, int $B_{k}$ are pairwise disjoint as well. It follows that the characteristic sphere $S_{p q}$ bounds the open $n$-ball

$$
\bigcup_{i=1}^{k} \text { int } B_{i} \bigcup_{i=1}^{k} \text { int } D_{i} \stackrel{\text { def }}{=} B_{p q}^{n} \text {. }
$$

By Theorem 5.1, $M^{n}$ is homotopy equivalent to the $n$-torus $T^{n}$.

Due to Hsiang and Wall 29, any closed $n$-manifold $(n \geq 5)$ homotopy equivalent to a torus is homeomorphic to a torus. So to conclude the proof of item 1), it remains to consider the case $n=3$. By Lemma 2 in [51, $M^{3}$ is irreducible (i.e., every tamely embedded 2-sphere in $M^{3}$ bounds a ball) because the universal cover is homeomorphic to $\mathbb{R}^{3}$. Due to Stallings's theorem [55], if there is an epimorphism $\pi_{1}\left(M^{3}\right) \rightarrow \mathbb{Z}$ with kernel $\neq \mathbb{Z}_{2}$, then $M^{3}$ is a fiber bundle over $S^{1}$ with fiber a closed surface $T$. Obviously such an epimorphism exists, because $\pi_{1}\left(M^{3}\right)$ is isomorphic to $\mathbb{Z}^{3}$.

The homotopy exact sequence

$$
\pi_{3}\left(S^{1}\right) \rightarrow \pi_{2}(T) \rightarrow \pi_{2}\left(M^{3}\right) \rightarrow \pi_{2}\left(S^{1}\right) \rightarrow \pi_{1}(T) \rightarrow \pi_{1}\left(M^{3}\right) \rightarrow \pi_{1}\left(S^{1}\right) \rightarrow 0
$$

of the fiber bundle $\left(M^{3}, S^{1}, M^{3} \rightarrow S^{1}\right)$ is the following:

$$
0 \rightarrow \pi_{2}(T) \rightarrow \pi_{2}\left(M^{3}\right) \rightarrow 0 \rightarrow \pi_{1}(T) \rightarrow \mathbb{Z}^{3} \rightarrow \mathbb{Z}^{1} .
$$

Hence, $\pi_{2}(T)=\pi_{2}\left(M^{3}\right)=0$ and $\pi_{1}(T)$ is mapped injectively into the free abelian group $\pi_{1}\left(M^{3}\right)$, and thus $\pi_{1}(T)$ is free abelian as well. Since $\pi_{2}(T)=\pi_{2}\left(M^{3}\right)=0$, $T$ is the 2 -torus $T^{2}=S^{1} \times S^{1}$. 
Now $M^{3}$ is homeomorphic to the factor-space $\left(T \times \mathbb{R}^{1}\right) / g$, where $(m, x+1) \in$ $T \times \mathbb{R}^{1}$ is equivalent to $(g(m), x)$ under some homeomorphism $g: T \rightarrow T$. We can take three generators $a, b, c$ of $\pi_{1}\left(M^{3}\right)$ so that $a, b \in \pi_{1}(T)$ and the following relations hold:

$$
a b=b a, \quad g_{*}(a)=c^{-1} a c, \quad g_{*}(b)=c^{-1} b c,
$$

where $g_{*}: \pi_{1}(T) \rightarrow \pi_{1}(T)$ is the homomorphism induced by $g$ [24. Since $\pi_{1}(T)$ is abelian, we have $g_{*}(a)=a$ and $g_{*}(b)=b$. This implies that $g$ is isotopic to the identity of $T$. Thus, $M^{3}$ is homeomorphic to $T \times S^{1}=T^{2} \times S^{1}=T^{3}$. This concludes the proof of item (1).

Item 22) follows from item (1) of Theorem 5.1 By Corollary 6.2, there is at least one repelling periodic point inside of characteristic spheres. This concludes the proof.

Corollary 7.1. Suppose $f$ is a structurally stable diffeomorphism of a closed $n$ manifold $M^{n}(n \geq 3)$ having an orientable expanding attractor of codimension one $\Omega$. Then the spectral decomposition of $f$ consists of $\Omega$, a finite nonzero number of repelling periodic orbits of index $n$, and a finite number (maybe zero) of periodic saddle orbit of index $n-1$.

Corollary 7.2. Suppose $f$ is a structurally stable diffeomorphism of a closed $n$ manifold $M^{n}(n \geq 3)$ having an orientable expanding attractor $\Omega$ of codimension one. Let $S_{p q}$ be a characteristic sphere corresponding to a 2-bunch $B=W^{u}(p) \cup$ $W^{u}(q)$ of $\Omega$, where $p, q \in \Omega$ are associated boundary periodic points. Then $S_{p q}$ bounds an $n$-ball containing $k_{0}+1$ repelling periodic points $\alpha_{1}, \ldots, \alpha_{k_{0}+1}\left(k_{0} \geq 0\right)$ of index $n$ and $k_{0}$ saddle periodic points $P_{1}, \ldots, P_{k}$ of index $n-1$. Moreover, the following conditions hold:

1) The union

$$
a_{p q}=\{p\} \cup W_{\emptyset}^{s}(p) \cup \alpha_{1} \cup W^{s}\left(P_{1}\right) \cup P_{1} \cup \alpha_{2} \cup \ldots \cup P_{k} \cup \alpha_{k+1} \cup W_{\emptyset}^{s}(q) \cup\{q\}
$$

is a compact arc with no self-intersections and with the endpoints $p, q$.

2) On the arc $a_{p q}$, the repelling periodic points of index $n$ alternate with the saddle periodic points of index $n-1$ (including $p$ and $q$ ).

Corollary 7.3. Suppose $f$ is an A-diffeomorphism of a closed $n$-manifold $M^{n}$ $(n \geq 3)$ having an orientable expanding attractor $\Omega$ of codimension one. Then the following conditions imply that $f$ is not structurally stable:

1) $f$ has a nontrivial basic set different from $\Omega$.

2) $M^{n}$ is not homotopy equivalent to the $n$-torus.

Now we consider non-orientable codimension one expanding attractors and contracting repellers. Recall that structurally stable diffeomorphisms with non-orientable codimension one expanding attractors exist on any closed 2-manifolds beginning with the 2-sphere [44. In contrast, we show that if a closed 3-manifold $M^{3}$ admits a non-orientable codimension one expanding attractor, then $M^{3}$ must be non-orientable. The following theorem, proved by V. Medvedev and E. Zhuzhoma, is a crucial step in the proof of Theorem 7.3.

Theorem 7.2. Let $f: M^{3} \rightarrow M^{3}$ be an A-diffeomorphism of a closed 3-manifold $M^{3}$. If $f$ has a non-orientable codimension one expanding attractor $\Omega$, then $M^{3}$ is non-orientable. 
Proof. Due to Theorem 2.1 of [45], $\Omega$ has 1-bunches. Let $W^{u}\left(m_{0}\right) \subset \Omega$ be an unstable manifold forming a 1-bunch of $\Omega$, where $m_{0}$ is a boundary periodic point. Without loss of generality we can assume that $m_{0}$ is a fixed point of $f$. According to Lemma 2.1 and Lemma 1.11 there is a homeomorphism $\varphi: W^{u}\left(m_{0}\right) \rightarrow W^{u}\left(m_{0}\right)$ such that $\varphi^{2}=i d$ and $m_{0}$ is the only fixed point of $\varphi$. It is convenient to prove the theorem in steps. The end of the proof of a step will again be denoted by $\diamond$.

Take a point $x_{0} \in W^{u}\left(m_{0}\right)$ such that $x_{0} \neq m_{0}$. Let $D_{\alpha} \subset W^{u}\left(m_{0}\right)-m_{0}$ be the family of disks ( $\alpha$ ranges over the interval $[0 ; 1]$ ) with the following properties:

- $x_{0} \in D_{0}, \varphi\left(x_{0}\right) \notin D_{0}, \varphi\left(x_{0}\right) \in D_{1}, D_{0} \cap \varphi\left(D_{0}\right)=\emptyset$.

- $D_{\alpha_{1}} \subset D_{\alpha_{2}}$ whenever $\alpha_{1}<\alpha_{2}$.

- The boundary $\partial D_{\alpha}$ varies continuously with respect to $\alpha$.

Such a family exists, because $x_{0} \neq \varphi\left(x_{0}\right)$ and $W^{u}\left(m_{0}\right)$ is homeomorphic to $\mathbb{R}^{2}$.

Step 7.1. There exists an index $\alpha_{0} \in(0 ; 1)$ such that

$$
\emptyset \neq D_{\alpha_{0}} \cap \varphi\left(D_{\alpha_{0}}\right)=\partial D_{\alpha_{0}} \cap \partial\left(\varphi\left(D_{\alpha_{0}}\right)\right) .
$$

Proof of Step 7.1] If $\alpha_{1}<\alpha_{2}$, then $\varphi\left(D_{\alpha_{1}}\right) \subset \varphi\left(D_{\alpha_{2}}\right)$, because $\varphi$ is a homeomorphism. Since $D_{0} \cap \varphi\left(D_{0}\right)=\emptyset$, we have that $D_{\alpha} \cap \varphi\left(D_{\alpha}\right)=\emptyset$ for $\alpha$ sufficiently small. On the other hand, $D_{\alpha_{1}} \cap \varphi\left(D_{\alpha_{1}}\right) \neq \emptyset$, because $\varphi\left(x_{0}\right) \in D_{1}$. Since $\partial D_{\alpha}$ varies continuously with respect to $\alpha, \partial \varphi\left(D_{\alpha}\right)=\varphi\left(\partial D_{\alpha}\right)$ varies continuously with respect to $\alpha$ as well. Hence there exists $\alpha_{0} \in(0 ; 1)$ such that the disks $D_{\alpha_{0}}, \varphi\left(D_{\alpha_{0}}\right)$ have a non-empty intersection at points of the boundaries only.

Step 7.2. There exists a curve $C \subset W^{u}\left(m_{0}\right)-m_{0}$, with no self-intersections, such that $C \cap \varphi(C)=\left\{x_{0}, \varphi\left(x_{0}\right)\right\}$, where $x_{0}, \varphi\left(x_{0}\right)$ are the endpoints of $C$.

Proof of Step 7.2 According to step 7.1, there is a point $x_{1} \in \partial D_{\alpha_{0}} \cap \varphi\left(\partial D_{\alpha_{0}}\right)$ for some $\alpha_{0} \in(0,1)$. Since $\varphi^{2}=i d$ and $m_{0}$ is the only fixed point of $\varphi$, it follows that $\varphi\left(x_{1}\right) \in \partial D_{\alpha_{0}} \cap \varphi\left(\partial D_{\alpha_{0}}\right)$ and $\varphi\left(x_{1}\right) \neq x_{1}$. There is the path $C_{01} \subset D_{\alpha_{0}}$ with no self-intersections such that $C_{01}$ connects the points $x_{0}, x_{1}$ (because $D_{\alpha_{0}}$ is a disk). Due to step [7.1] the interior of $D_{\alpha_{0}}$ and the image of this interior under $\varphi$ have no intersections. Therefore, $\varphi\left(C_{01}\right) \subset \varphi\left(D_{\alpha_{0}}\right)$ is a path with no self-intersections. Moreover, $\varphi\left(C_{01}\right)$ connects $\varphi\left(x_{0}\right)$ and $\varphi\left(x_{1}\right)$, and $C_{01} \cap \varphi\left(C_{01}\right)=\emptyset$. Obviously, the curve $\varphi\left(C_{01}\right)$ does not divide the disk $\varphi\left(D_{\alpha_{0}}\right)$. Hence there is a path $C_{12}$ with no self-intersections that connects the points $\varphi\left(x_{0}\right)$ and $x_{1}$. Moreover, we can assume that $\varphi\left(C_{01}\right) \cap C_{12}=\varphi\left(x_{0}\right)$. By definition, put $C=C_{01} \cup C_{12}$. Since $\varphi^{2}=i d$, it follows that $C \cap \varphi(C)=\left\{x_{0}, \varphi\left(x_{0}\right)\right\}$.

Step 7.3. The set $M_{c}=\bigcup_{x \in C}(x, y)_{\emptyset}^{s}$ is homeomorphic to the open Möbius band. Moreover, any arc $(x, y)_{\emptyset}^{s}$ is transversal (in the topological sense) to the middle line of $M_{c}$.

Proof of Step 7.9 Let us consider the open rectangle $P=\bigcup_{x \in C-x_{0}}(x, y)_{\emptyset}^{s}$. Then $M_{c}$ is obtained by adding to $P$ the $\operatorname{arc}\left(x_{0}, \varphi\left(x_{0}\right)\right)_{\emptyset}^{s}$. The relation $\varphi^{2}=i d$ implies that this addition can be represented as attaching two opposite sides of the closed rectangle clos $P$ by an orientation-reversing homeomorphism. Hence, $M_{c}$ is homeomorphic to the open Möbius band. By construction, any arc $(x, y)_{\emptyset}^{s}$ is transversal (in the topological sense) to the middle line of the Möbius band.

By step [7.2, the union $C \cup \varphi(C)=c_{0}$ is a simple closed curve in $W^{u}\left(m_{0}\right)$. Therefore, $c_{0}$ bounds on $W^{u}\left(m_{0}\right)$ the open disk $D_{c}$. 
Step 7.4. $m_{0} \in D_{c}$.

Proof of Step 7.4 By construction, $\varphi\left(c_{0}\right)=c_{0}$. Since $\varphi$ is a homeomorphism, it follows that $\varphi\left(D_{c}\right)=D_{c}$ and $\varphi\left(c_{0} \cup D_{c}\right)=c_{0} \cup D_{c}$, because the exterior of $c_{0}$ on $W^{u}\left(m_{0}\right)$ is homeomorphic to an open annulus. Hence, $\varphi$ has a fixed point in $c_{0} \cup D_{c}$. Since $m_{0}$ is the unique fixed point of $\varphi, m_{0} \in D_{c}$.

Step 7.5. There exists an open subset $C^{3} \subset M^{3}$ that is homeomorphic to the product $M_{b} \times(0,1)$, where $M_{b}$ is the open Möbius band.

Proof of Step 7.5. We keep the notation of the steps above. Take an open simple arc $l_{0} \subset D_{c}$ such that $l_{0} \cap \varphi\left(l_{0}\right)=\emptyset$ and one of endpoints is $x_{0}$. Because $\varphi\left(x_{0}\right) \neq x_{0}$ and $\varphi$ is a homeomorphism, such an arc exists. Since $\varphi(C) \cap C$ consists of only two points $x_{0}$ and $\varphi\left(x_{0}\right)$, it follows that there is an open strip $P_{1} \subset D_{c}$, bounded by the curve $C$ and $\operatorname{arcs} l_{0}$ and $\varphi\left(l_{0}\right)$, such that $\varphi\left(P_{1}\right) \cap P_{1}=\emptyset$ and $m_{0} \notin \varphi\left(P_{1}\right) \cup P_{1}$. Therefore, $\varphi\left(P_{1}\right)$ is the open strip bounded by the curve $\varphi(C)$ and the $\operatorname{arcs} l_{0}$ and $\varphi\left(l_{0}\right)$. As a consequence, the union $P=\varphi\left(l_{0}\right) \cup l_{0} \cup \varphi\left(P_{1}\right) \cup P_{1}$ is an open strip in $D_{c}$. Moreover, one component of the boundary $\partial P$ coincides with the boundary $\partial D_{c}=\varphi(C) \cup C$ of $D_{c}$. Let $C_{1}$ be the side of $P_{1} \subset D_{c}$ which is opposite the side $C \stackrel{\text { def }}{=} C_{0}$. Note that the other pair of opposite sides $l_{0}$ and $\varphi\left(l_{0}\right)$ is invariant under $\varphi$. Therefore there exists a splitting of the closed rectangle $\varphi\left(l_{0}\right) \cup l_{0} \cup P_{1} \cup C_{0} \cup C_{1}=$ clos $P_{1}$ into disjoint $\operatorname{arcs} C_{\alpha}$ ( $\alpha$ ranges over the interval $[0,1]$ ) such that endpoints of each arc map onto each other by $\varphi$. Moreover, without loss of generality we can assume that the arcs $C_{\alpha}, 0 \leq \alpha \leq 1$, form a topological foliation on $\operatorname{clos} P_{1}$. It follows from the equality $\varphi\left(P_{1}\right) \cap P_{1}=\emptyset$ that, given any $\alpha \in[0,1], C_{\alpha} \cup \varphi\left(C_{\alpha}\right)$ is a closed simple curve. As a consequence, the family $C_{\alpha} \cup \varphi\left(C_{\alpha}\right), \alpha \in[0,1]$, forms the topological foliation on $P$ with leaves $C_{\alpha} \cup \varphi\left(C_{\alpha}\right)$ that are closed simple curves invariant under $\varphi$. Therefore, $C_{\alpha} \cup \varphi\left(C_{\alpha}\right)$ is the boundary of the set $M_{\alpha}=\bigcup_{x \in C_{\alpha} \cup \varphi\left(C_{\alpha}\right)}(x, \varphi(x))_{\emptyset}^{s}$, which is homeomorphic to the open Möbius band $M_{b}$, step [7.3] Since stable manifolds are pairwise disjoint, the $M_{\alpha}$ are pairwise disjoint as well.

The theorem on the continuous dependence of invariant manifolds on initial conditions implies that the sets $M_{\alpha}$ vary continuously with respect to $\alpha$. Since the strip $P$ is homeomorphic to the product $S^{1} \times(0,1)$, it follows that $C^{3}=$ $\bigcup_{x \in P}(x, \varphi(x))_{\emptyset}^{s}=\bigcup_{\alpha \in(0,1)} M_{\alpha}$ is homeomorphic to the product $M_{b} \times(0,1) . \diamond$

It is well known that the Möbius band is a non-orientable surface. Hence, $C^{3}$ is a non-orientable 3-manifold. As a consequence, $M^{3}$ is a non-orientable 3 -manifold as well [24]. This concludes the proof.

Theorem 7.3. Let $f: M^{3} \rightarrow M^{3}$ be a structurally stable diffeomorphism of a closed 3-manifold $M^{3}$. Then the spectral decomposition of $f$ contains neither codimension one non-orientable expanding attractors nor codimension one non-orientable contracting repellers. In particular, $f$ has no codimension one Plykin attractors.

Proof. Assume the converse. Without loss of generality we can assume that $f$ has a codimension one non-orientable expanding attractor $\Omega$. According to Theorem 7.2 $M^{3}$ is non-orientable. Let $\tilde{M}^{3}$ be the orientable manifold such that $p: \tilde{M}^{3} \rightarrow M^{3}$ is a double covering for $M^{3}$. Then there exists a diffeomorphism $\tilde{f}: \tilde{M}^{3} \rightarrow \tilde{M}^{3}$ which is a lift of $f$, i.e., $f \circ p=p \circ \tilde{f}$. It is easy to see that $\tilde{f}$ is an $A$-diffeomorphism. Since $p$ is a local diffeomorphism, $\tilde{f}$ satisfies the strong transversality condition as well. By Robinson's theorem [47], $\tilde{f}$ is a structurally stable diffeomorphism. The 
preimage $p^{-1}(\Omega)$ contains a codimension one attractor $\tilde{\Omega}$, because $\Omega$ is an attractor. Since $\Omega$ is locally homeomorphic to the product of a Cantor set and $\mathbb{R}^{2}$, so is $\tilde{\Omega}$. It follows that $\tilde{\Omega}$ is an expanding attractor of codimension one.

Recall that $\tilde{M}^{3}$ is orientable. Due to Theorem $7.2 \tilde{\Omega}$ is orientable and has no 1-bunches. By Corollary 1.3, $\tilde{\Omega}$ has 2-bunches only.

Since $\Omega$ is non-orientable, it follows that there is an open component $R \subset M^{3}-\Omega$ such that the accessible boundary of $R$ contains the 1-bunch $W^{u}\left(m_{0}\right) \subset \Omega$, where $m_{0}$ is a boundary periodic point. Without loss of generality we can assume that $m_{0}$ is a fixed point of $f$. Let $\tilde{m}_{1} \in \tilde{\Omega}$ be the lift under $p$ of the point $m_{0}$. Because $p$ is a double covering, $\tilde{m}_{1}$ is a boundary periodic point of $\tilde{\Omega}$. Since every bunch of $\tilde{\Omega}$ is a 2 -bunch, it follows that there is a boundary periodic point $\tilde{m}_{2} \in \tilde{\Omega}, \tilde{m}_{2} \neq \tilde{m}_{1}$, associated with $\tilde{m}_{1}$.

By Corollary 7.2 , there are $k+1 \geq 1$ repelling periodic points $\tilde{\alpha}_{1}, \ldots, \tilde{\alpha}_{k+1}$ and $k \geq 0$ saddle periodic points $P_{1}, \ldots, P_{k}$ of the index 2 such that the following conditions hold:

- $a_{12}=\left\{\tilde{m}_{1}\right\} \cup W_{\emptyset}^{s}\left(\tilde{m}_{1}\right) \cup \tilde{\alpha}_{1} \cup W^{s}\left(P_{1}\right) \cup P_{1} \cup \tilde{\alpha}_{2} \cup \ldots \cup P_{k} \cup \tilde{\alpha}_{k+1} \cup W_{\emptyset}^{s}\left(\tilde{m}_{2}\right) \cup\left\{\tilde{m}_{2}\right\}$ is a compact arc with no self-intersections.

- On the arc $a_{12}$, the repelling periodic points $\tilde{\alpha}_{i}$ alternate with the saddle periodic points $P_{i}$.

By Lemma 2.1. if $\tilde{x}_{n} \rightarrow \tilde{m}_{1}$ as $n \rightarrow \infty$, then $\tilde{y}_{n} \rightarrow \tilde{m}_{2}$, where $\tilde{x}_{n} \in W^{u}\left(\tilde{m}_{1}\right)$, $\tilde{y}_{n} \in W^{s}\left(\tilde{m}_{2}\right)$, and $\left(\tilde{x}_{n}, \tilde{y}_{n}\right)^{s}=\left(\tilde{x}_{n}, \tilde{y}_{n}\right)_{\emptyset}^{s}$. Since $\left(\tilde{x}_{n}, \tilde{y}_{n}\right)^{s} \cup \tilde{\Omega}=\emptyset$ and $p(\tilde{\Omega})=\Omega$,

$$
p\left(\left(\tilde{x}_{n}, \tilde{y}_{n}\right)_{\emptyset}^{s}\right)=\left(p\left(\tilde{x}_{n}\right), p\left(\tilde{y}_{n}\right)\right)_{\emptyset}^{s} .
$$

The inclusion $p\left(\tilde{x}_{n}\right) \in W^{u}\left(m_{0}\right)$ implies $p\left(\tilde{y}_{n}\right) \in W^{u}\left(m_{0}\right)$, because $W^{u}\left(m_{0}\right)$ is a 1 -bunch. Since $p$ is a continuous map, $p\left(\tilde{x}_{n}\right) \rightarrow p\left(\tilde{m}_{1}\right)=m_{0}$ and $p\left(\tilde{y}_{n}\right) \rightarrow p\left(\tilde{m}_{2}\right)$. From the inclusion $p\left(\tilde{y}_{n}\right) \in W^{u}\left(m_{0}\right)$ and Lemma 2.1, it follows that $p\left(\tilde{y}_{n}\right) \rightarrow m_{0}$. Hence, $p\left(\tilde{m}_{2}\right)=m_{0}$.

The relation $p \circ f=\tilde{f} \circ p$ implies that $p$ maps the invariant manifolds of $\tilde{f}$ onto the invariant manifolds of $f$. Then

$$
\begin{gathered}
p\left(W_{\emptyset}^{s}\left(\tilde{m}_{1}\right)\right)=p\left(W_{\emptyset}^{s}\left(\tilde{m}_{2}\right)\right), \quad p\left(\tilde{\alpha}_{1}\right)=p\left(\tilde{\alpha}_{k+1}\right), \\
p\left(W^{s}\left(P_{1}\right)\right)=p\left(W^{s}\left(P_{k}\right)\right), \quad p\left(P_{1}\right)=p\left(P_{k}\right), \quad p\left(\tilde{\alpha}_{2}\right)=p\left(\tilde{\alpha}_{k}\right), \ldots .
\end{gathered}
$$

Since the repelling periodic points $\tilde{\alpha}_{i}$ alternate with the saddle periodic points $P_{i}$ on the $\operatorname{arc} a_{12}$, we see that the number of periodic points on $a_{12}$ is odd, because the endpoints are of saddle type. As a consequence, there is either a periodic point $\tilde{\alpha}_{i}$ with $p\left(W^{s}\left(P_{i-1}\right)\right)=p\left(W^{s}\left(P_{i}\right)\right)$ or a periodic point $P_{i}$ with $p\left(W_{1}^{s}\left(P_{i}\right)\right)=p\left(W_{2}^{s}\left(P_{i}\right)\right)$, where $W_{1}^{s}\left(P_{i}\right), W_{2}^{s}\left(P_{i}\right)$ are different components of $W^{s}\left(P_{i}\right)-P_{i}$. In both cases, there is a point $\left(\tilde{\alpha}_{i}\right.$ or $\left.P_{i}\right)$ at which $p$ is not a local homeomorphism. This contradiction concludes the proof.

\section{TOPOLOGICAL CLASSIFICATION}

After Theorem 7.1, it is natural to classify structurally stable diffeomorphisms with expanding orientable attractors of codimension one on the $n$-torus $T^{n}, n \geq 3$. Let $f: T^{n} \rightarrow T^{n}$ be such a diffeomorphism. Denote by $f_{*}: H_{1}\left(T^{n}, \mathbb{R}^{n}\right) \rightarrow$ $H_{1}\left(T^{n}, \mathbb{R}^{n}\right)$ the automorphism of the first homology group $H_{1}\left(T^{n}, \mathbb{R}^{n}\right) \simeq \mathbb{R}^{n}$ induced by $f$. The automorphism $f_{*}$ is hyperbolic if $f_{*}$ has no eigenvalues of absolute value 1 . The following theorem was proved in [19], thm. 1, with no assumption on the structural stability. 
Theorem 8.1. Suppose $f: T^{n} \rightarrow T^{n}$ is a structurally stable diffeomorphism having an orientable expanding attractor $\Omega$ of codimension one. Then $f_{*}$ is hyperbolic.

Following [12, let us call a diffeomorphism $A: M^{n} \rightarrow M^{n}$ a $\pi_{1}$-diffeomorphism if, given any homeomorphism $g: K \rightarrow K$ of a compact CW complex and any map $h: K \rightarrow M^{n}$ such that $A_{*} h_{*}=h_{*} g_{*}$, there is a unique base-point-preserving map $h^{\prime}: K \rightarrow M^{n}$, homotopic to $h$, such that $A \circ h^{\prime}=h^{\prime} \circ g$.

Let $f: T^{n} \rightarrow T^{n}$ be a structurally stable diffeomorphism having an orientable expanding attractor $\Omega$ of codimension one. Due to Theorem $8.1 f_{*}$ is hyperbolic. Take an algebraic automorphism $A(f): T^{n} \rightarrow T^{n}$ such that $f_{*}=A(f)_{*}$. By Proposition 2.1 in [12], $A(f)_{*}$ is a $\pi_{1}$-diffeomorphism. Then there exists a map $h: T^{n} \rightarrow T^{n}$, homotopic to the identity, such that $h \circ f=A(f) \circ h$. By definition, put

$$
P(f, h)=\left\{x \in T^{n} \mid h^{-1}(x) \text { contains at least two points }\right\} .
$$

Lemma 8.1. The map $h$ is continuous and satisfies the following conditions:

- $h(\Omega)=T^{n}$.

- If $\left\{p_{i}, q_{i}\right\}_{i=1}^{k}$ are the pairs of associated boundary periodic points of $f$, then $h\left(p_{i}\right)=h\left(q_{i}\right)$. Moreover, $h\left(p_{i}\right)=h\left(q_{i}\right)$ is a periodic point of $A(f)$ for each $i=1, \ldots, k$.

- $P(f, h)=\bigcup_{i=1}^{k} W^{u}\left(p_{i}\right) \cup W^{u}\left(q_{i}\right)$.

- Suppose $K_{i}$ is a component of $M^{n}-\Omega$; then $h\left(K_{i}\right)$ is the unstable manifold $W^{u}\left(h\left(p_{i}\right)\right)=W^{u}\left(h\left(q_{i}\right)\right)$ of $A(f)$, where $p_{i}, q_{i}$ are associated boundary periodic points such that $\delta\left(K_{i}\right)=W^{u}\left(p_{i}\right) \cup W^{u}\left(p_{i}\right)$. Moreover, $h\left(K_{i} \cup \delta\left(K_{i}\right)\right)=$ $W^{u}\left(h\left(p_{i}\right)\right)$.

- If $\breve{\Omega} \subset \Omega$ is a union of unstable manifolds that do not contain boundary periodic points, then the restriction $h_{\breve{\Omega}}$ is a homeomorphism onto its image $h(\breve{\Omega})$.

Proof. The proof is similar to that of Theorem 2 in [19] and Lemma 4 in [20]. We omit the details.

The relation $h \circ f=A(f) \circ h$ and Lemma 8.1 imply that $A(f)$ is a codimension one Anosov diffeomorphism.

Given any pair $(p, q)$ of associated boundary periodic points, we assign the number $n(p, q) \in \mathbb{N}$ of repelling periodic points that are inside the characteristic sphere $S_{p q}$ corresponding to the 2-bunch $B=W^{u}(p) \cup W^{u}(q)$. This number is well defined, because it does not depend on the choice of a characteristic sphere. Obviously, $n(p, q)=n\left(f^{m}(p), f^{m}(q)\right)$ for any $m \in \mathbb{Z}$. Therefore we can assign the number $n(p, q) \stackrel{\text { def }}{=} n(O(p, q))$ to the pair of orbits $O(p), O(q)$ of the points $p, q$.

Let $\left\{O\left(p_{i}, q_{i}\right)\right\}_{i=1}^{k}$ be the pairs of orbits of associated boundary points, and let $\left\{n\left(O\left(p_{i}, q_{i}\right)\right)\right\}_{i=1}^{k}$ be the set of corresponding natural numbers defined above. To each periodic orbit $h\left(O\left(p_{i}\right)\right)=h\left(O\left(q_{i}\right)\right)$ of $A(f)$, we assign the number $n\left(O\left(p_{i}, q_{i}\right)\right)$. The collection $\left\{h\left(O\left(p_{i}\right)\right), n\left(O\left(p_{i}, q_{i}\right)\right)\right\}_{i=1}^{k}$, denoted by $\mathcal{D}(f, h)$, is called the data set of $f$,

$$
\mathcal{D}(f, h)=\left\{h\left(O\left(p_{i}\right)\right), n\left(O\left(p_{i}, q_{i}\right)\right)\right\}_{i=1}^{k} .
$$

Let $A$ be an arbitrary codimension one hyperbolic automorphism of $T^{n}$, and let $\left\{O_{j}\right\}_{j=1}^{r}$ be any finite family of periodic orbits of $A$. To any orbit $O_{j}$, let us assign an arbitrary natural number $n_{j} \in \mathbb{N}$. The collection $\left\{O_{j}, n_{j}\right\}_{j=1}^{r}$ is called an 
admissible data set of the automorphism A. Note that by Theorem 7.1 a data set of $f$ is admissible whenever $f$ is structurally stable.

Suppose $\left\{O_{j}^{1}, n_{j}^{1}\right\}_{j=1}^{r_{1}}$ and $\left\{O_{j}^{2}, n_{j}^{2}\right\}_{j=1}^{r_{2}}$ are admissible data sets of codimension one hyperbolic automorphisms $A_{1}$ and $A_{2}$ respectively. These data sets are called equivalent if $r_{1}=r_{2} \stackrel{\text { def }}{=} r$ and there is an affine transformation $\psi: T^{n} \rightarrow T^{n}$ (i.e., the composition of an automorphism and translation, $\psi=A x+\xi)$ such that

$$
\psi \circ A_{1}=A_{2} \circ \psi, \quad \psi\left(\bigcup_{j=1}^{r} O_{j}^{1}\right)=\bigcup_{j=1}^{r} O_{j}^{2}, \quad n\left(\psi\left(O_{j}\right)\right)=n\left(O_{j}\right), 1 \leq j \leq r .
$$

Theorem 8.2. Suppose $f_{1}, f_{2}: T^{n} \rightarrow T^{n}$ are structurally stable diffeomorphisms having orientable expanding attractors of codimension one $\Omega_{1}$ and $\Omega_{2}$ respectively. Then $f_{1}$ and $f_{2}$ are conjugate if and only if the data sets $\mathcal{D}\left(f_{1}, h_{1}\right)$ and $\mathcal{D}\left(f_{2}, h_{2}\right)$ are equivalent, where $h_{i}: T^{n} \rightarrow T^{n}(i=1,2)$ are continuous maps homotopic to the identity and such that $h_{i} \circ f_{i}=A_{i} \circ h_{i},\left(f_{i}\right)_{*}=\left(A_{i}\right)_{*}$.

Proof. The proof is similar to the proof of Theorem 3 of [19] and Theorem 2 of [20], where the conjugacy was proved in the basins of the codimension one expanding attractors. Note that in those theorems, there was no assumption on structural stability of the diffeomorphisms $f_{1}, f_{2}$ (see also Theorem 2.5 of [45] and Theorem 4 of [63]). The proof is omitted.

Theorem 8.3. Let $A$ be a codimension one hyperbolic automorphism of $T^{n}$ such that the stable manifolds of $A$ are one-dimensional. Given an admissible data set $\left\{O_{j}, n_{j}\right\}_{j=1}^{r}$ of $A$, there is a structurally stable diffeomorphism $f: T^{n} \rightarrow T^{n}$ having an orientable expanding attractor of codimension one and such that $\mathcal{D}(f, h)=$ $\left\{O_{j}, n_{j}\right\}_{j=1}^{r}$, where $h: T^{n} \rightarrow T^{n}$ is a continuous map homotopic to the identity with $h \circ f=A \circ h$ and $f_{*}=A_{*}$.

Proof. Actually, $f$ is constructed by the 'surgery operation' described by Smale 53. and Williams [59] to get a $D A$-diffeomorphism with a one-dimensional basic set on the 2-torus. The neat construction is in [31] and [48] (for the 2-torus), and 13] for the 3 -torus, and [45] (for any $n$-torus, $n \geq 2$ ). According to Theorem 2.3 in [45], there is a structurally stable $D A$-diffeomorphism $f^{\prime}: T^{n} \rightarrow T^{n}$ having a unique expanding orientable codimension one attractor $\Omega^{\prime}$ and the data set $\left\{O_{j}, 1\right\}_{j=1}^{r}$. By a slight modification of $f^{\prime}$ inside the characteristic spheres of $\Omega^{\prime}$, one can get the desired diffeomorphism $f$ with the data set $\left\{O_{j}, n_{j}\right\}_{j=1}^{r}$ and the expanding orientable codimension one attractor $\Omega=\Omega^{\prime}$.

Theorems 8.2 and 7.3 imply the following corollary.

Corollary 8.1. Suppose $f_{1}, f_{2}: T^{3} \rightarrow T^{3}$ are structurally stable diffeomorphisms having expanding attractors of codimension one $\Omega_{1}$ and $\Omega_{2}$ respectively. Then $f_{1}$ and $f_{2}$ are conjugate if and only if the data sets $\mathcal{D}\left(f_{1}, h_{1}\right)$ and $\mathcal{D}\left(f_{2}, h_{2}\right)$ are equivalent, where $h_{i}: T^{3} \rightarrow T^{3}(i=1,2)$ are continuous maps homotopic to the identity and such that $h_{i} \circ f_{i}=A_{i} \circ h_{i}$ and $\left(f_{i}\right)_{*}=\left(A_{i}\right)_{*}$.

\section{ACKNOWLEDGMENTS}

The authors are grateful to D. Anosov, Ch. Bonatti, V. Medvedev, R. Plykin, P. Schweitzer, and R. Williams for useful discussions. We thank the referee for many valuable suggestions. 


\section{REFERENCES}

[1] D. Anosov. Geodesic flows on closed Riemannian manifolds of negative curvature. Trudy Mat. Inst. Steklov. 90 (1967); English transl., Proc. Steklov Inst. Math. 90 (1969). MR 36:7157

[2] D. Anosov. On a class of invariants sets of smooth dynamical systems. Proc. Fifth Internat. Conference of nonlinear oscillations. Vol. 2, Izdanie Inst. Mat. Akad Nauk Ukrain. SSR, Kiev, 1970, pp. 39-45 (in Russian). MR 47:8969

[3] S. Aranson, G. Belitsky, E. Zhuzhoma. Introduction to Qualitative Theory of Dynamical Systems on Closed Surfaces. Transl. of Math. Monographs, v. 153, 1996, Amer. Math. Soc. Providence, Rhode Island. MR 97c:58135

[4] S. Aranson, V. Grines. Topological classification of cascades on closed two-dimensional manifolds. Russian Math. Surveys, 45(1990), no 1, 1-35. MR 91d:58195

[5] S. Aranson, R. Plykin, A. Zhirov, E. Zhuzhoma. Exact upper bounds for the number of one-dimensional basic sets of surface A-diffeomorphisms. Journ. of Dyn. and Contr. Syst., 3(1997), no 1, 1-18. MR 98d:58109

[6] S. Aranson, E. Zhuzhoma. On the structure of quasiminimal sets of foliations on surfaces. Russian Acad. Sci. Sb. Mat. 82(1995), 397-424. MR 95i:57027

[7] Ch. Bonatti, R. Langevin. Difféomorphismes de Smale des surfaces. Astérisque, 250(1998). MR 99m:58123

[8] R. Bowen. Periodic points and measures for axiom A diffeomorphisms. Trans. Amer. Math. Soc., 154(1971), 377-397. MR 43:8084

[9] M. Brown. A proof of the generalized Schoenflies theorem. Bull. Amer. Math. Soc. 66(1960), no 2, 74-76. MR 22:8470b

[10] M. Brown, H. Gluck. Stable structure on manifolds. Ann. of Math. 79(1964), no 1, 1-58. MR 28:1608a

[11] F. Farrell, L. Jones. New attractors in hyperbolic dynamics. J. Diff. Geom. 15(1980), 107-133. MR 82h:58025

[12] J. Franks. Anosov diffeomorphisms. Global Analisys. Proc. Symp. in Pure Math., AMS 14(1970), 61-94. MR 42:6871

[13] J. Franks, C. Robinson. A quasi-Anosov diffeomorphism that is not Anosov. Trans. Amer. Math. Soc. 223(1976), 267-278. MR 54:11399

[14] V. Grines. On topological equivalence of one-dimensional basic sets of diffeomorphisms on two-dimensional manifolds. Uspekhi Mat. Nauk 29(1974), no. 6, 163-164 (in Russian). MR 55:13498

[15] V. Grines. On topological conjugacy of diffeomorphisms of a two-dimensional manifold onto one-dimensional orientable basic sets I. Trans. Moscow Math. Soc. 32(1975), 31-56. MR 54:6203

[16] V. Grines. On topological conjugacy of diffeomorphisms of a two-dimensional manifold onto one-dimensional orientable basic sets II. Trans. Moscow Math. Soc. 34(1977), 237-245. MR 57:14057

[17] V. Grines Structural stability and asymptotic behavior of invariant manifolds of $A$-diffeomorphisms of surfaces. Journal of Dyn. and Control Syst. 3(1997), no 1, 91-110. MR 98b:58095

[18] V. Grines On the topological classification of structurally stable diffeomorphisms of surfaces with one-dimensional attractors and repellers. Sborn. Math. 188(1997), no 4, 537-569. MR 98g:58092

[19] V. Grines, E. Zhuzhoma. The topological classification of orientable attractors on an n-dimensional torus. Russian Math. Surveys 34 (1978), no. 4, 163-164. MR 80k:58071

[20] V. Grines, E. Zhuzhoma. Necessary and sufficient conditions for the topological equivalence of orientable attractors on $n$-dimensional torus. Diff. and Integr. Equat. Sb. of Gorky University, 1981, 89-93 (in Russian). MR 84k:58136

[21] V. Grines, E. Zhuzhoma. Structuraly stable diffeomorphisms with codimension one basic sets. Preprint. Universite de Bourgogne, Laboratoire de Topologie, Dijon Cedex, 2000, no 223.

[22] J. Guckenheimer. Endomorphisms of Riemann sphere. Global Analisys. Proc. Symp. in Pure Math., AMS 14(1970), 95-123. MR 43:500 
[23] A. Haefliger. Variétés feuilletées. Ann. Scuola Norm. Sup. Pisa 16(1962), 367-397. MR 32:6487

[24] J. Hempel. 3-manifolds. Princeton, Annals Math. Studies, 86(1976). MR 54:3702

[25] K. Hiraide. A simple proof of the Franks-Newhouse theorem on codimension one Anosov diffeomorphisms. Ergod. Th. and Dynam. Sys., 21(2001), 801-806. MR 2002e:37042

[26] M. Hirsch. Differential Topology. Sringer-Verlag, 1976. MR 56:6669

[27] M. Hirsch, C. Pugh. Stable manifolds and hyperbolic sets. Global Analysis, Proc. Symp. Pure Math., Amer. Math. Soc., 14(1970), 133-163. MR 42:6872

[28] M. Hirsch, J. Palis, C. Pugh, M. Shub. Neighborhoods of hyperbolic sets. Invent. Math. 9(1970), 121-134. MR 41:7232

[29] W. C. Hsiang, C. T. C. Wall. On homotopy tori, II. Bull.London Math. Soc. 1(1969), 341-342. MR 41:2691

[30] L. Jones. Locally strange hyperbolic sets. Trans. Amer. Math. Soc. 275(1983), no 1, 153162. MR 84b:58085

[31] A. Katok, B. Hasselblatt. Introduction to the Modern Theory of Dynamical Systems. Encyclopedia of Math. and its Appl., Cambridge Univ. Press, 1994. MR 96c:58055

[32] H. Kollmer. On hyperbolic attractors of codimension one. Lect. Notes Math. 597(1977), 330-334. MR 57:7687

[33] H. Lawson. Foliations. Bull. Amer. Math. Soc. 80(1974), 369-418. MR 49:8031

[34] W. Magnus, A. Karrass, D. Solitar. Combinatorial Group Theory: Presentations of groups in terms of generators and relations. Interscience Publishers, 1966.

[35] R. Mañé. A proof of $C^{1}$ stability conjecture. Publ. Math. IHES 66(1988), 161-210. MR 89e:58090

[36] B. Mazur. On embedding of spheres. Bull. Amer. Math. Soc. 65(1959), 59-65. MR 22:8469

[37] S. E. Newhouse. On codimension one Anosov diffeomorphisms. Amer. J. Math. 92(1970), no 3, 761-770. MR 43:2741

[38] Z. Nitecki. Differentiable dynamics. MIT Press, Cambridge, 1971. MR 58:31210

[39] S. P. Novikov. Topology of foliations. Trans. Moscow Math. Soc. 14 (1965), 268-304 (1967).

[40] J. Palis. On Morse-Smale dynamical systems. Topology 8(1969), no 4, 385-404. MR 39:7620

[41] J. Palis, W. de Melo. Geometric Theory of Dynamical Systems. An Introduction. SpringerVerlag, 1982. MR 84a:58004

[42] J. Plante. The homology class of an expanded invariant manifold. Lect. Notes Math. 468(1975), 251-256. MR 58:31269

[43] R. V. Plykin. On the topology of basic sets of Smale diffeomorphisms. Math. USSRSbornik 13 (1971), 297-307. MR 44:3348

[44] R. V. Plykin. Sources and sinks of A-diffeomorphisms of surfaces. Math. USSR-Sbornik 23(1974), 223-253.

[45] R. V. Plykin. The geometry of hyperbolic attractors of smooth cascades. Russian Math. Surveys 39 (1984), no. 6, 85-131. MR 86f:58106

[46] G. Reeb. Sur certaines propriétés topologiques des variétés feuilletées. Actual. Sci. Ind., no 1183, Hermann, Paris, 1952. MR 14:1113a

[47] C. Robinson. Structural stability of $C^{1}$ diffeomorphisms. J. Diff. Equat. 22(1976), no 1, 28-73. MR 57:14051

[48] C. Robinson. Dynamical Systems: stability, symbolic dynamics, and chaos. Studies in Adv. Math., 2nd ed., CRC Press, Boca Raton, FL, 1999. MR 2001k:37003

[49] C. Robinson, R. Williams. Finite stability is not generic. Dynamical Systems: Proc. Symp. (Brazil, 1971), Academic Press, New York, London, 1973, 451-462. MR 48:9763

[50] C. Robinson, R. Williams. Classification of expanding attractors: an example. Topology 15(1976), 321-323. MR 54:3762

[51] H. Rosenberg. Foliations by planes. Topology 7(1968), 131-138. MR 37:3595

[52] T. B. Rushing. Topological Embeddings. Acad. Press, NY and London, 1973. MR 50:1247

[53] S. Smale. Differentiable dynamical systems. Bull. AMS, 73(1967), 747-817. MR 37:3598

[54] N. Steenrod. The topology of fibre bundles. Princeton, New Jersey, 1951. MR 12:522b

[55] J. Stallings. On fibering certain 3-manifolds. Topology of 3-manifolds, Prentice-Hall, 1962. MR 28:1600

[56] D. Sullivan, R. Williams. On the homology of attractors. Topology 15(1976), 259-262. MR 54:1304 
[57] I. Tamura. Topology of Foliations. Iwanami Shoten, Tokyo, 1976; Russian transl., "Mir", Moscow, 1979; English transl., Amer. Math. Soc., 1992. MR 84a:57028a MR 84a:57028b, MR 93c:57021

[58] R. Williams. One-dimensional nonwandering sets. Topology, 6(1967), 473-487. MR 36:897

[59] R. Williams. The 'DA' maps of Smale and structural stability, Global Anal., Proc. Symp. Pure. Math., AMS 14(1970), 329-334. MR 41:9296

[60] R. Williams. Expanding attractors. Publ. Math. I.H.E.S. 43(1974), 169-203. MR 50:1289

[61] A. Yu. Zhirov. Enumeration of hyperbolic attractors on orientanle surfaces and applications to pseudo-Anosov homeomorphisms. Russ. Acad. Sci. Dokl. Math., 47(1993). MR 94i:58152

[62] A. Yu. Zhirov. Complete combinatorial invariants for conjugacy of hyperbolic attractors of diffeomorphisms of surfaces. Journ. Dyn. and Contr. Syst., 6(2000), no 3, 397-430. MR 2001g:37051

[63] E. Zhuzhoma. Orientable basic sets of codimension 1. Soviet Math. (Iz. VUZ) 26(1982), no 5, 17-25. MR 83i:58077

Department of Mathematics, Agriculture Academy of Nizhny Novgorod, 97 Gagarin Ave, Nizhny Novgorod, 603107 Russia

Department of Applied Mathematics, Nizhny Novgorod State Technical University, 24 Minina Str., Nizhny Novgorod, 603600 Russia

E-mail address: zhuzhoma@mail.ru 